\title{
THE NEXT GENERATION VIRGO CLUSTER SURVEY (NGVS). I. INTRODUCTION TO THE SURVEY*
}

\author{
Laura Ferrarese $^{1}$, Patrick Côté ${ }^{1}$, Jean-Charles Cuillandre $^{2}$, S. D. J. Gwyn ${ }^{1}$, Eric W. Peng ${ }^{3,4}$, Lauren A. MacArthur ${ }^{1}$, \\ Pierre-Alain Duc ${ }^{5}$, A. Boselli ${ }^{6}$, Simona Mei ${ }^{7,8}$, Thomas Erben ${ }^{9}$, Alan W. McConnachie $^{1}$, Patrick R. Durrell ${ }^{10}$, \\ J. Christopher Mihos ${ }^{11}$, Andrés Jordán ${ }^{12}$, Ariane Lançon ${ }^{13}$, Thomas H. Puzia ${ }^{12}$, Eric Emsellem ${ }^{14,15}$,

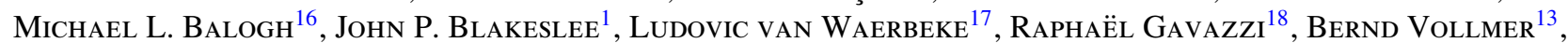 \\ J. J. Kavelaars ${ }^{1}$, David Woods ${ }^{17}$, Nicholas M. Ball ${ }^{1}$, S. Boissier $^{6}$, Stéphane Courteau ${ }^{19}$, E. Ferriere ${ }^{5,8}$, G. GavazZi ${ }^{20}$, \\ Hendrik Hildebrandt ${ }^{17}$, P. Hudelot ${ }^{18}$, M. Huertas-Company ${ }^{7,8}$, Chengze Liu $^{21}$, Dean McLaughlin $^{22}$, Y. Mellier ${ }^{18}$, \\ Martha Milkeraitis ${ }^{17}$, David Schade ${ }^{1}$, Chantal Balkowski ${ }^{7,8}$, Frédéric Bournaud ${ }^{5}$, R. G. Carlberg ${ }^{23}$, S. C. Chapman ${ }^{24}$, \\ Henk Hoekstra $^{25}$, Chien Peng ${ }^{26}$, Marcin Sawicki $^{27}$, Luc Simard ${ }^{1}$, James E. Taylor $^{28}$, R. Brent Tully $^{29}$, Wim van Driel ${ }^{7}$, \\ Christine D. Wilson ${ }^{30}$, Todd Burdullis ${ }^{2}$, Billy Mahoney ${ }^{2}$, And Nadine Manset ${ }^{2}$ \\ ${ }^{1}$ Herzberg Institute of Astrophysics, National Research Council of Canada, Victoria, BC, V9E 2E7, Canada \\ ${ }^{2}$ Canada-France-Hawaii Telescope Corporation, Kamuela, HI 96743, USA \\ ${ }^{3}$ Department of Astronomy, Peking University, Beijing 100871, China \\ ${ }^{4}$ Kavli Institute for Astronomy and Astrophysics, Peking University, Beijing 100871, China \\ ${ }^{5}$ AIM Paris Saclay, CNRS/INSU, CEA/Irfu, Université Paris Diderot, Orme des Merisiers, F-91191 Gif sur Yvette cedex, France \\ ${ }^{6}$ Laboratoire d'Astrophysique de Marseille, UMR 6110 CNRS, 38 rue F. Joliot-Curie, F-13388 Marseille, France \\ ${ }^{7}$ GEPI, Observatoire de Paris, CNRS, 5 Place Jules Jannssen, F-92195 Meudon, France \\ ${ }^{8}$ Bureau des Galaxies, Etoiles, Physique, Instrumentation, Université Paris Denis Diderot, F-75205, Paris Cedex 13, France \\ ${ }^{9}$ Argelander-Institut für Astronomie, University of Bonn, Auf dem Hügel 71, D-53121 Bonn, Germany \\ ${ }^{10}$ Department of Physics and Astronomy, Youngstown State University, Youngstown, OH, USA \\ ${ }^{11}$ Department of Astronomy, Case Western Reserve University, Cleveland, OH, USA \\ 12 Departamento de Astronomía y Astrofísica, Pontificia Universidad Católica de Chile, 7820436 Macul, Santiago, Chile \\ 13 Observatoire Astronomique, Université de Strasbourg \& CNRS UMR 7550, 11 rue de l'Université, F-67000 Strasbourg, France \\ ${ }^{14}$ Université de Lyon 1, CRAL, Observatoire de Lyon, 9 av. Charles André, F-69230 Saint-Genis Laval; CNRS, UMR 5574; ENS de Lyon, France \\ 15 European Southern Observatory, Karl-Schwarzchild-Str. 2, D-85748 Garching, Germany \\ 16 Department of Physics and Astronomy, University of Waterloo, Waterloo, Ontario, N2L 3G1, Canada \\ ${ }^{17}$ Department of Physics and Astronomy, University of British Columbia, 6224 Agricultural Road, Vancouver, B.C., V6T 1Z1, Canada \\ ${ }^{18}$ Institut d'Astrophysique de Paris, UMR 7095 CNRS \& UPMC, 98bis Bd Arago, F-75014 Paris, France \\ 19 Department of Physics, Engineering Physics and Astronomy, Queen's University, Kingston, ON, Canada \\ ${ }^{20}$ Dipartimento di Fisica, Universita degli Studi di Milano-Bicocca, Piazza della Scienza 3, I-20126 Milano, Italy \\ ${ }^{21}$ Department of Physics, Shangai Jiao Tong University, 800 Dongchuan Road, Shangai 200240, China \\ 22 Astrophysics Group, Lennard-Jones Laboratories, Keele University, Keele, Staffordshire ST5 5BG, UK \\ ${ }^{23}$ Department of Astronomy and Astrophysics, University of Toronto, Toronto, ON M5S 3H4, Canada \\ 24 Institute of Astronomy, University of Cambridge, Madingley Road, Cambridge CB3 OHA, UK \\ ${ }^{25}$ Leiden University, Leiden Observatory, Niels-Bohrweg 2, 2333CA, Leiden, the Netherlands \\ 26 The Observatoires of the Carnegie Institution for Science, 813 Santa Barbara Street, Pasadena, CA 91101, USA \\ ${ }^{27}$ Department of Astronomy and Physics, St. Mary's University, Halifax, NS B3H 3C3, Canada \\ ${ }^{28}$ Department of Physics \& Astronomy, 200 University Avenue West, Waterloo, Ontario, N2L 3G1, Canada \\ ${ }^{29}$ Institute for Astronomy, University of Hawaii, 2680 Woodlawn Dr., Honolulu, HI 96822, USA \\ ${ }^{30}$ Department of Physics and Astronomy, McMaster University, Hamilton, ON L8S 4M1, Canada \\ Received 2011 November 3; accepted 2012 March 27; published 2012 May 1
}

\begin{abstract}
The Next Generation Virgo Cluster Survey (NGVS) is a program that uses the $1 \mathrm{deg}^{2} \mathrm{MegaCam}$ instrument on the Canada-France-Hawaii Telescope to carry out a comprehensive optical imaging survey of the Virgo cluster, from its core to its virial radius — covering a total area of $104 \mathrm{deg}^{2}$ - in the $u^{*}$ griz bandpasses. Thanks to a dedicated data acquisition strategy and processing pipeline, the NGVS reaches a point-source depth of $g \approx 25.9$ mag $(10 \sigma)$ and a surface brightness limit of $\mu_{g} \sim 29$ mag $\operatorname{arcsec}^{-2}$ ( $2 \sigma$ above the mean sky level), thus superseding all previous optical studies of this benchmark galaxy cluster. In this paper, we give an overview of the technical aspects of the survey, such as areal coverage, field placement, choice of filters, limiting magnitudes, observing strategies, data processing and calibration pipelines, survey timeline, and data products. We also describe the primary scientific topics of the NGVS, which include: the galaxy luminosity and mass functions; the color-magnitude relation; galaxy scaling relations; compact stellar systems; galactic nuclei; the extragalactic distance scale; the large-scale environment of the cluster and its relationship to the Local Supercluster; diffuse light and the intracluster medium; galaxy interactions and evolutionary processes; and extragalactic star clusters. In addition, we describe a number of ancillary programs dealing with "foreground" and "background" science topics, including the study of highinclination trans-Neptunian objects; the structure of the Galactic halo in the direction of the Virgo Overdensity and Sagittarius Stream; the measurement of cosmic shear, galaxy-galaxy, and cluster lensing; and the identification of distant galaxy clusters, and strong-lensing events.
\end{abstract}

Key words: galaxies: clusters: individual (Virgo) - galaxies: distances and redshifts - galaxies: general - galaxies: luminosity function, mass function - galaxies: photometry - galaxies: star clusters: general

Online-only material: color figures 


\section{INTRODUCTION}

Understanding the growth of structure-including galaxies on all mass scales-remains one of the central goals of modern astrophysics. During the past decade, extraordinary efforts have been devoted to cosmological surveys of the distant/early universe (including studies of the cosmic microwave background, baryon acoustic oscillations, cosmic shear, and largescale structure, as well as searches for high- $z$ supernovae, galaxies, and quasars). These surveys have led to dramatic improvements in our understanding of hierarchical structure formation within a cold dark matter (CDM) dominated universe. $\Lambda \mathrm{CDM}$ cosmological parameters have now been measured with high precision, leading to advances in our understanding of the initial conditions that governed the growth of structures on all scales (e.g., Spergel et al. 2003, 2007; Cole et al. 2005; Tegmark et al. 2006; Percival et al. 2007). These achievements have been mirrored by advances in the speed and precision of numerical methods used to simulate the hierarchical formation of structures over wide ranges in mass and radius (e.g., Springel et al. 2005, 2008; Diemand et al. 2007; Navarro et al. 2010). Careful comparisons have now shown that, for simulations containing dark matter (DM) only, different codes and initial conditions produce results that are highly consistent on the scales of galaxies or smaller, with convergence achieved down to scales of less than $0.5 \%$ of the virial radius (Power et al. 2003; Navarro et al. 2004; Klypin et al. 2011; Boylan-Kolchin et al. 2009).

However, the situation becomes considerably more complicated when baryons are added to the models (e.g., Gnedin et al. 2004; Kravtsov et al. 2005; Nagai \& Kravtsov 2005). Simulating from first principles the diverse stellar systems identified in the local universe-which range in scales from rich clusters down to very low mass dwarf galaxies and star clusters-hinges on the treatment of complex and often poorly understood physical processes such as baryon condensation and adiabatic contraction, accretion and mergers, gas cooling, star formation, stellar/ active galactic nucleus (AGN) feedback, and the ejection of baryons. In light of this complexity, progress in this area is certain to proceed in close conjunction with observations of stellar systems in complete and unbiased surveys, as these data can be used to test and refine the models and their input physics. Such observations most often take the form of surveys of galaxies and clusters in the high- and intermediate-redshift universe, which give an integrated, statistical picture of structure evolution over cosmic time. An equally powerful and complementary approach is to focus on stellar systems in the local universe, which can be studied at a level of detail that is unattainable for more distant objects.

Amassing large-and representative-samples of galaxies can present a challenge for the latter approach, as it requires large areas of the sky to be surveyed. This problem can be mitigated by dedicated surveys that target regions of the local volume that are "overdense" (i.e., cluster or group environments). The Virgo cluster, as the richest cluster of galaxies in the nearby universe (see, e.g., Tully 1982; Klypin et al. 2003), is an obvious first target for a survey of the low-redshift galaxy population. In this paper, which is the first in an extended series, we describe a

\footnotetext{
* Based on observations obtained with MegaPrime/MegaCam, a joint project of CFHT and CEA/DAPNIA, at the Canada-France-Hawaii Telescope (CFHT), which is operated by the National Research Council (NRC) of Canada, the Institut National des Science de l'Univers of the Centre National de la Recherche Scientifique (CNRS) of France and the University of Hawaii.
}

comprehensive optical imaging survey of the Virgo cluster using the $1 \mathrm{deg}^{2}$ MegaCam instrument on the Canada-France-Hawaii Telescope (CFHT). This Next Generation Virgo Cluster Survey (NGVS) is designed to serve as the definitive census of stellar systems in the Virgo cluster, at least until the next generation of dedicated, wide-field imaging telescopes on the ground and in space comes online at the end of this decade. The NGVS will therefore provide a key data set for testing and refining simulations of galaxies and galaxy clusters in the low-redshift universe.

This paper is organized as follows. In Section 2, we present a brief overview of the Virgo cluster, including its basic properties and large-scale environment. The technical aspects of the NGVS, including the observing and mapping strategies, filter choices, exposure times, and survey limits, are discussed in Section 3, while details of the data processing and calibrations and the distribution of data products are given in Section 4. In Section 5, we discuss the scientific motivations for the survey, which include not just Virgo-specific science (e.g., the luminosity and mass functions, galaxy evolution and the color-magnitude relation (CMR), scaling relations, compact stellar systems, galactic nuclei, the extragalactic distance scale, diffuse light, galaxy interactions, and star clusters), but also foreground (i.e., the outer solar system and Galactic halo) and background studies (i.e., cosmic shear, high- $z$ galaxy clusters, and strong lenses). Section 6 gives a brief description of a number of imaging and spectroscopic programs that capitalize on the census of Virgo globular clusters (GCs), compact objects, and Galactic halo stars enabled by the NGVS. We summarize in Section 7.

\section{THE VIRGO CLUSTER}

At a distance of $16.5 \pm 0.1$ (random) \pm 1.1 (systematic) Mpc (Mei et al. 2007), the Virgo cluster is the richest cluster of galaxies nearest to our own Galaxy. ${ }^{31}$ With a gravitating mass in the range of $\mathcal{M}_{200}=(1.4-4.2) \times 10^{14} \mathcal{M}_{\odot}$ (McLaughlin 1999; Urban et al. 2011; see also Nulsen \& Böhringer 1995; Schindler et al. 1999), Virgo is also the dominant mass concentration in the local universe, marking (by convention) the center of the Local Supercluster (de Vaucouleurs 1953, 1958, 1961a; see also Klypin et al. 2003). Early efforts to characterize the rich population of galaxies within the cluster include those of Shapley \& Ames (1926, 1929a, 1929b, 1929c, 1929d, 1929e), Hubble \& Humason (1931), Smith (1936), Zwicky (1942, 1957), Holmberg (1958), de Vaucouleurs (1961a, 1961b), Sandage (1972), and Reaves (1956, 1962, 1983). Such efforts culminated with the series of papers in the 1980s by Binggeli, Sandage, and Tammann, who imaged a $\approx 140 \mathrm{deg}^{2}$ area centered on the cluster core using blue-sensitive photographic plates taken with the $2.5 \mathrm{~m}$ du Pont telescope at Las Campanas Observatory (see Section 2.1 below). Their catalog contains 2096 galaxies within the survey region (Binggeli et al. 1985), of which 1850 were considered by them to be certain or probable cluster members. This rich system of galaxies-lying at nearly the same distance and encompassing virtually all known morphological types - has since played a central role in shaping our understanding of how galaxies form and evolve in dense environments. Indeed, the Virgo cluster is probably the most thoroughly studied cluster of galaxies in the universe, and

\footnotetext{
31 The much less massive Fornax and Ursa Major clusters lie at distances of $20.0 \mathrm{Mpc}$ and $\approx 17.1-18.6 \mathrm{Mpc}$, respectively (Blakeslee et al. 2009; Tully \& Pierce 2000; Tully et al. 2009).
} 


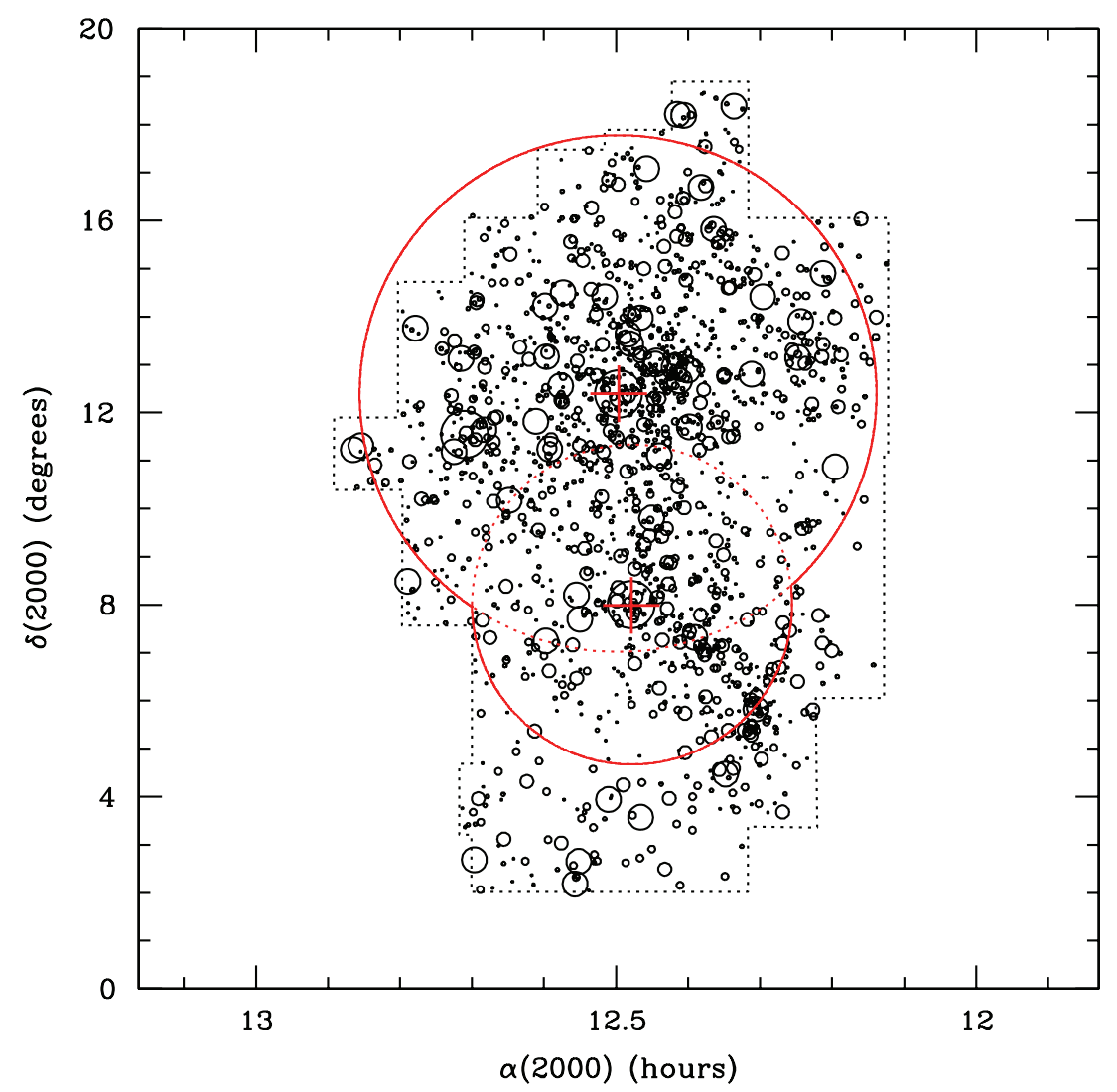

Figure 1. Spatial distribution of galaxies from the Virgo Cluster Catalog (VCC; Binggeli et al. 1985) classified as certain or probable cluster members (including galaxies originally classified as non-members in the VCC, but subsequently shown to have a velocity $\left.\lesssim 3000 \mathrm{~km} \mathrm{~s}^{-1}\right)$, for a total of 1762 galaxies. The symbol size scales with the galaxy $B$-band luminosity. The dotted lines indicate the boundaries of the Binggeli et al. (1985) survey. The red crosses indicate the location of M87 and M49, which are located close to the respective centers of the A and B subclusters (Binggeli et al. 1987, 1993), and the dotted red circles indicate their virial radii (see Section 3 for details). The area surveyed by the NGVS is the region interior to the solid red curves, a total area of $104 \mathrm{deg}^{2}$.

(A color version of this figure is available in the online journal.)

remains the obvious target for a modern, digital survey of stellar systems in the low-redshift universe.

\subsection{The Virgo Cluster Catalog (VCC)}

Although it is more than a quarter century old, the widefield photographic survey of Binggeli and coworkers still serves as the standard reference for the Virgo cluster in the optical. Beginning in the late 1970 s, these investigators capitalized on the wide-field imaging capabilities of the $2.5 \mathrm{~m}$ du Pont reflector (a $1.5 \times 1.5$ field with $50 \times 50 \mathrm{~cm}$ plates) to carry out a moderately deep (45-75 minute exposures) photographic survey of Virgo down to an extended-source (galaxy) completeness limit of $B_{\text {lim }} \approx 18 \mathrm{mag}$. For the purpose of identifying the lowest surface brightness dwarf galaxies, the du Pont imaging was supplemented with scans of long-exposure IIIaJ plates taken with the $48^{\prime \prime}$ Schmidt at Palomar, each of which covered a $6^{\circ} \times 6^{\circ}$ field. Overall, the limiting magnitude for point sources in their survey was estimated to lie in the range $B_{\text {lim }} \approx 22$ to $\approx 23$ depending on the plate material (Binggeli et al. 1985). Plate-toplate image quality varied significantly over the survey, ranging between 0.5 and 2".0 (Binggeli et al. 1984).

Results from this survey were published in a series of papers on the Virgo cluster and its constituent galaxies. Binggeli et al. (1984) presented intensity profiles and photometric and structural parameters for 109 "standard" galaxies near the center of the cluster. Sandage \& Binggeli (1984) presented an illustrated atlas of Virgo dwarf galaxies and introduced a classification scheme for these low-mass systems. A related atlas for spiral galaxies was presented in Sandage et al. (1985). Their final Virgo Cluster Catalog (VCC) of 2096 galaxies was presented in Binggeli et al. (1985), which consisted of homogenized identifications, coordinates, membership assessments, morphological types, isophotal diameters, axial ratios, and radial velocity measurements (compiled from the literature). The galaxy luminosity function in the Virgo cluster, including its dependence on morphological type, was discussed in Sandage et al. (1985), while the morphological and kinematic structure of the cluster was examined in Binggeli et al. (1987). The VCC survey boundaries are shown by the dashed black lines in Figure 1, while the circles indicate the location of galaxies classified as certain or probable members by Binggeli et al. (1985).

The VCC has had a profound impact on a remarkably wide range of astrophysical questions, such as the form of the galaxy luminosity function, the photometric and structural properties of galaxies, the abundance of high and low surface brightness galaxies, the extragalactic distance scale, the nature of galactic nuclei, the galaxy-intracluster medium (ICM) connection, the morphology-density relation, the dynamics and virialization of the Virgo cluster (and clusters in general), and the distribution of DM. Indeed, the VCC continues to serve as the departure point for innumerable, multi-wavelength studies of the Virgo cluster and its constituent galaxies (see Section 2.2).

Despite its obvious legacy, the VCC is out of date by modern standards, and a large number of ongoing or planned surveys of the Virgo cluster at longer and shorter wavelengths are 
now compromised by the lack of high-quality, complementary optical and near-infrared (NIR) data for this benchmark cluster. To some extent, this situation has been mitigated by the Sloan Digital Sky Survey (SDSS), whose survey area encompasses the entire Virgo cluster region. Indeed, a growing number of studies of the Virgo cluster that rely on SDSS imaging (and spectroscopy) have begun to appear in the literature (e.g., Lisker et al. 2006a, 2006b, 2007, 2008; Rines \& Geller 2008; Janz \& Lisker 2008, 2009; McDonald et al. 2009, 2011; Kim et al. 2010; Chen et al. 2010). This is to be expected since the SDSS offers several distinct advantages relative to the VCC: panoramic imaging with linear (CCD) detectors, complete coverage in five independent bands (ugriz), and the ability for investigators to retrieve digital SDSS images directly. At the same time, it is important to recall that the largest and most extended galaxies present a considerable challenge for the SDSS pipeline (see, e.g., the discussion in Chen et al. 2010) and that the SDSS has not improved substantially on the VCC point-source and surface brightness detection limits, i.e., $g_{\lim } \sim 23.2(5 \sigma)$ and $25 \lesssim\left\langle\mu_{g}\right\rangle_{e} \lesssim 26 \mathrm{mag} \mathrm{arcsec}^{-2}$ for the SDSS (York et al. 2000; Kniazev et al. 2004) versus $B_{\text {lim }} \sim 22-23$ and $\left\langle\mu_{B}\right\rangle_{e} \sim 25.3 \mathrm{mag} \mathrm{arcsec}^{-2}$ for the VCC. This similarity in depth is the result of the identical telescope apertures $(2.5 \mathrm{~m})$ and comparable image quality (median SDSS seeing $\sim 1$ ". 4 FWHM), while the superior efficiency of the SDSS CCDs is nearly canceled by the much shorter exposure times (54 s) relative to the VCC.

\subsection{Multi-wavelength Surveys of the Virgo Cluster}

Clearly, there is a need for a wide-field optical imaging survey of the Virgo cluster that capitalizes on the superior efficiency, linearity, and depth achievable with modern large field of view CCD mosaic cameras on $4 \mathrm{~m}$ class telescopes. First and foremost, such a survey would address a wide-ranging array of open questions in astrophysics, as we detail below (see Section 5). At the same time, an optical survey that improves significantly over the VCC or SDSS would also leverage the many programs and surveys that have targeted Virgo at shorter and longer wavelengths. Here, we briefly review some of the noteworthy surveys that fall into this category. Although this list is far from complete, it serves to illustrate the multi-wavelength appeal of the Virgo cluster in modern astrophysics. Figure 2 illustrates the overlap of several programs that have targeted the Virgo cluster; we also note that a comprehensive multiwavelength database of dynamical, photometric, and structural parameters for over 2000 optically selected galaxies in the Virgo cluster is maintained by the GOLDMine project (Gavazzi et al. 2003). ${ }^{32}$

In the radio, coverage of the Virgo cluster is available at $1.4 \mathrm{GHz}$ as part of the $10,000 \mathrm{deg}^{2}$ Very Large Array (VLA) Faint Images of the Radio Sky at Twenty centimeters survey (FIRST; Becker et al. 1995; White et al. 1997) with an angular resolution of $5^{\prime \prime}$, and by the NRAO VLA Sky Survey (NVSS; Condon et al. 1998) with an angular resolution of $45^{\prime \prime}$. Perhaps most relevant for the NGVS, because of its higher sensitivity and spatial resolution, is the Arecibo Legacy Fast ALFA Survey (ALFALFA; Giovanelli et al. 2005; Kent 2008; Haynes et al. 2011). ALFALFA is a blind extragalactic $\mathrm{H}$ i survey that aims to detect more than 30,000 $\mathrm{H}$ I line sources within $z \sim 0.06$. While the full ALFALFA survey covers $\sim 7000 \mathrm{deg}^{2}$ to a sensitivity of $2.2 \mathrm{mJy}$ per $3 . .5$ beam, the Virgo cluster falls squarely within

\footnotetext{
32 http://goldmine.mib.infn.it
}

its survey region; ALFALFA has already produced a large number of $\mathrm{H}$ I sources in this direction, mostly identified with VCC galaxies (Gavazzi et al. 2008), but also including some "optically inert" candidates (e.g., Kent et al. 2007, 2009; Haynes et al. 2007; Koopmann et al. 2008; Haynes 2008; Kent 2010). Deeper Arecibo H I observations for two fields in Virgo covering $15 \mathrm{deg}^{2}$ to a sensitivity of $0.6 \mathrm{mJy}$ beam $^{-1}$ will soon be available from the Arecibo Galaxy Environment Survey (AGES; J. E. Taylor et al. 2012, in preparation), while higher resolution 15" VLA H I data for 53 late-type galaxies is available from the VLA Imaging of Virgo Spirals in Atomic Gas survey (VIVA; Chung et al. 2009a). At submillimeter wavelengths, targeted observations of molecular gas distributions include ${ }^{12} \mathrm{CO}(J=$ 1-0) emission line maps of 28 Virgo cluster spiral galaxies obtained with the Five College Radio Astronomy Observatory (FCRAO) $14 \mathrm{~m}$ telescope at 45" resolution (Chung et al. 2009b), and ${ }^{12} \mathrm{CO}(J=3-2)$ maps for 32 spiral galaxies obtain as part of the James Clerk Maxwell Telescope Nearby Galaxy Legacy Survey (JCMT; Wilson et al. 2009a, 2011) at 14.'5 resolution.

Moving to the far-infrared, surveys that targeted large areas of the Virgo cluster include the VIRGOFIR program (D. Fadda et al. 2012, in preparation) which used Spitzer to survey $\sim 30 \mathrm{deg}^{2}$ of the Virgo cluster at 24 and $70 \mu \mathrm{m}$, and the Herschel Virgo Cluster Survey (HeViCS; Davies et al. 2010, 2012), which will produce a fully sampled map, at five wavelengths between 100 and $600 \mu \mathrm{m}$, of $\sim 60 \mathrm{deg}^{2}$ in four partially overlapping Virgo fields. Targeted observations include 250, 350, and $500 \mu \mathrm{m}$ images for 82 Virgo galaxies obtained as part of the Herschel Reference Survey (HRS; Boselli et al. 2010) at an angular resolution of $\sim 30^{\prime \prime}$, and $6.75 \mu \mathrm{m}$ ( $6^{\prime \prime}$ resolution) and $15.0 \mu \mathrm{m}$ $\left(8^{\prime \prime}\right.$ resolution) images for 123 galaxies (mostly late-type galaxies brighter than $B_{T}=18$ mag and classified as cluster members in the VCC) observed with Infrared Space Observatory (ISO; Boselli et al. 2003).

At shorter wavelengths, the Two Micron All Sky Survey (2MASS; Skrutskie et al. 2006) covered the entire cluster in $J, H$, and $K_{s}$, while the Large Area Survey (LAS, part of the UKIRT Infrared Deep Sky Survey, or UKIDSS; Lawrence et al. 2007) covers an area of $4000 \mathrm{deg}^{2}$, and includes most of the cluster below a declination of $\delta_{2000} \sim+16^{\circ}$ in $Y, J, H$, and $K$. As for targeted NIR observations, $H$-band images for a magnitudelimited sample of $286 \mathrm{VCC}$ galaxies with $B_{T} \leqslant 16 \mathrm{mag}$ are part of the "deep Spectroscopic and $H$-band Imaging Survey of Virgo galaxies" (SHIVir; McDonald et al. 2009, 2011). SHIVir, which employed a variety of 2-4 m size telescopes and wide-field NIR detectors (CFHT/WIRCam, UKIRT/WFCAM, UH88in/ULBCam), represents the most extensive deep nearinfrared survey of Virgo cluster galaxies to date, reaching typical brightnesses $\mu_{\mathrm{H}} \sim 24 \mathrm{mag}_{\operatorname{arcsec}}{ }^{-2}$. Additional near-infrared pointed observations of large samples of Virgo galaxies are discussed in Boselli et al. (1997, 2000) and Gavazzi et al. (2000).

In the optical, the cluster is covered by the SDSS (York et al. 2000) in the ugriz bands; also to be mentioned here is the dedicated optical survey of the diffuse intracluster light (ICL) in Virgo carried out with the $0.6 \mathrm{~m}$ Burrell-Schmidt telescope (e.g., Mihos et al. 2005; Janowiecki et al. 2010; Rudick et al. 2010). Targeted optical observations of Virgo galaxies are of course numerous. Two surveys are particularly relevant for the study of early-type galaxies: the Advanced Camera for Surveys (ACS) Virgo Cluster Survey (ACSVCS; Côté et al. 2004) and ATLAS $^{3 \mathrm{D}}$ (Cappellari et al. 2011a). The ACSVCS obtained high-resolution F475W and F850LP imaging from Hubble Space Telescope (HST)/ACS for 100 Virgo early-type galaxies 


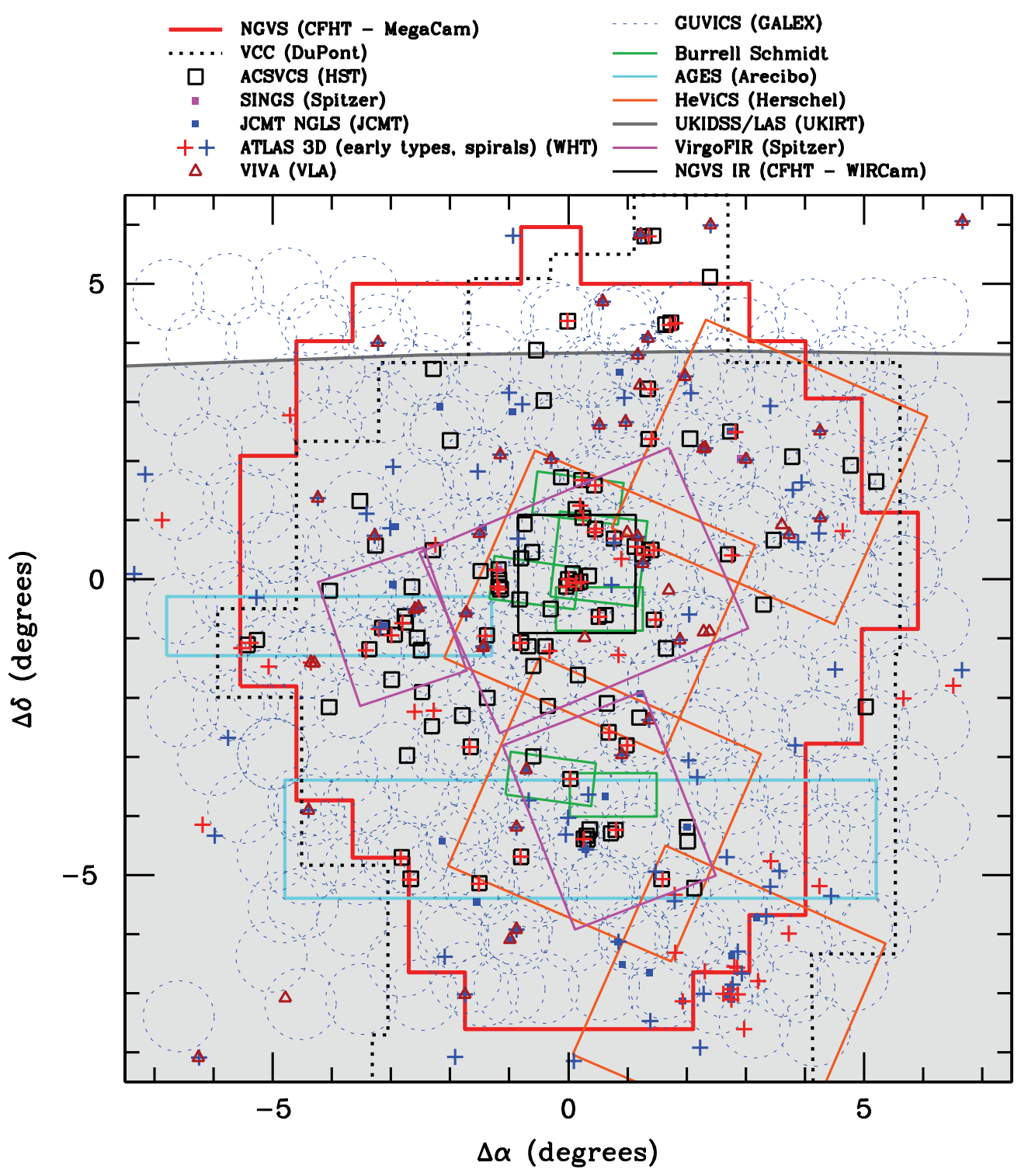

Figure 2. NGVS survey area, shown in heavy red, compared to the regions covered by a number of multi-wavelength surveys of the Virgo cluster (primarily at non-optical wavelengths). The different surveys are indicated in the legend at the top of the figure (the light gray area shows the region covered by UKIDSS/LAS; see the text for complete details). Complete coverage over the entire NGVS survey area is also available in the IR, optical, $21 \mathrm{~cm} \mathrm{H} \mathrm{I} \mathrm{line,} \mathrm{and} \mathrm{X-rays} \mathrm{from} 2 \mathrm{MASS}$, SDSS, ALFALFA, and ROSAT, respectively.

spanning a range of nearly three orders of magnitude in blue luminosity down to $\mathcal{L}=1.6 \times 10^{8} \mathcal{L}_{\odot, B}$. The ACSVCS sample forms the basis of follow-up X-ray programs with Chandra (the AGN Multi-wavelength Survey of Early-type Galaxies in the Virgo cluster: AMUSE-Virgo; Gallo et al. 2008, 2010) as well as $8.4 \mathrm{GHz}$ observations from the VLA (Capetti et al. 2009). $\operatorname{ATLAS}^{3 \mathrm{D}}$ targeted 260 early-type galaxies brighter than $\mathcal{L}=8.2 \times 10^{9} \mathcal{L}_{\odot, K}$. Of these, 58 galaxies are considered by Cappellari et al. (2011a) to be Virgo cluster members. For all of these galaxies, observations are available in the radio, millimeter, and optical domains, including multi-color imaging and two-dimensional (2D) spectroscopy. For late-type galaxies, notable optical surveys include narrowband $\mathrm{H} \alpha$ images for a (combined) sample of $\sim 200$ bright spiral galaxies and fainter blue compact dwarf galaxies by Koopmann et al. (2001), Boselli \& Gavazzi (2002), and Boselli et al. (2002).

At shorter wavelengths still, the NGVS, HeViCS, and ALFALFA surveys provided the motivation for a deep far- and near-ultraviolet Galaxy Evolution Explorer (GALEX) survey of Virgo, GUViCS. The survey is described in detail in Boselli et al. (2011). GUViCS was originally designed to cover a $120 \mathrm{deg}^{2}$ area extending slightly beyond the boundaries of the NGVS, to be imaged with both the NUV and FUV detectors. The FUV detector, however, became inoperative during the course of the survey (officially concluded in the summer of 2010), with the result that while the entire survey area was covered in the NUV band (for a total of 237 pointings), only $40 \%$ of the area was imaged in the FUV (for a total of 133 pointings). First results from GUViCS, focusing on the galaxy UV luminosity function in the central part of Virgo and on the effects of environment on H I scaling relations, are presented in Boselli et al. (2011) and Cortese et al. (2011).

Finally, X-ray coverage of the entire Virgo region was obtained as part of ROSAT's all-sky survey in the $0.1-2.4 \mathrm{keV}$ spectral range, with spatial resolution varying between $20^{\prime \prime}$ and 60" (Trümper 1993; Böhringer et al. 1994). 


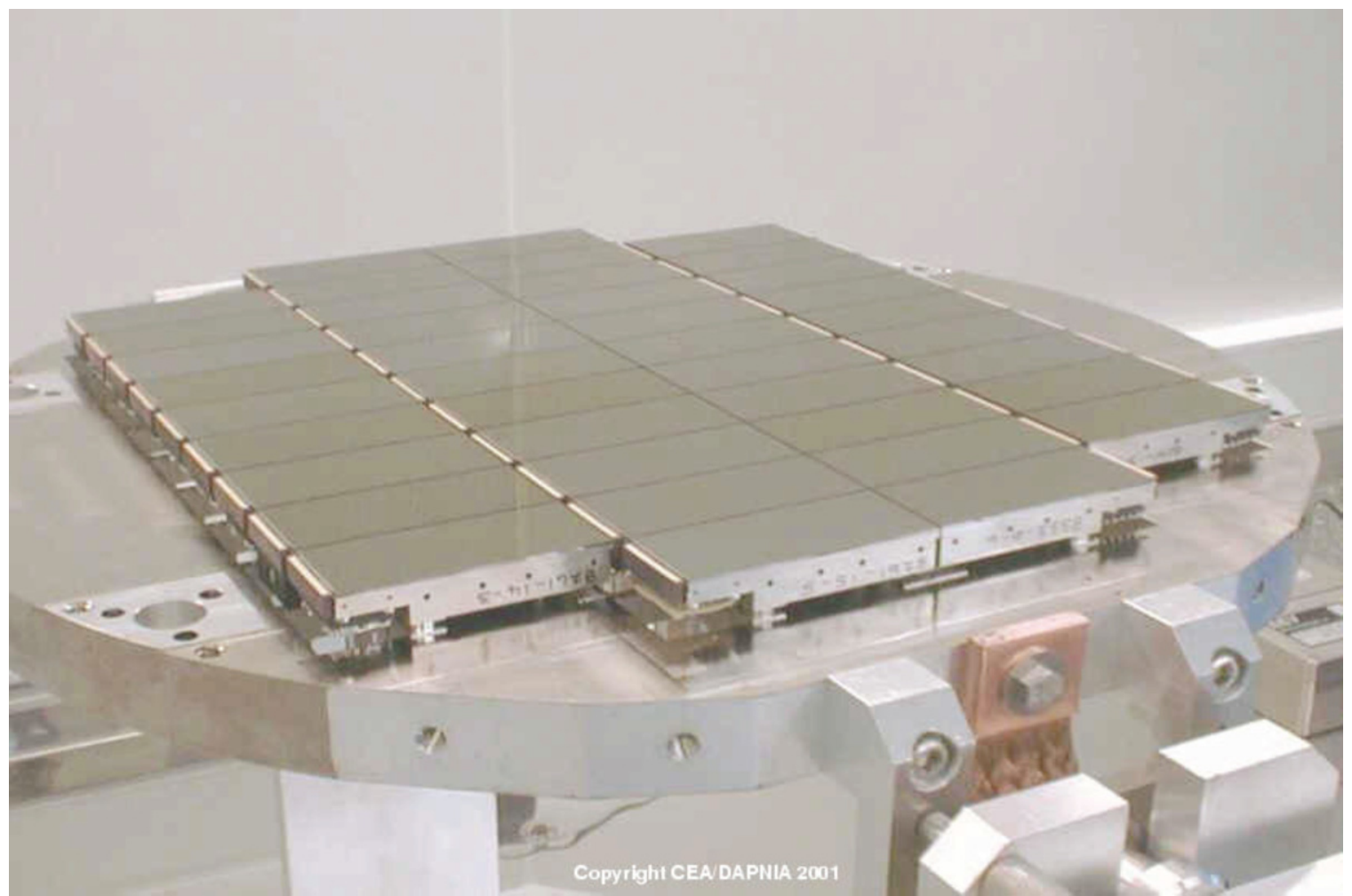

Figure 3. The $402048 \times 4612$ pixel CCDs that make up the MegaCam detector system (Boulade et al. 2003). Each MegaCam image covers an area of $0.96 \times 0.94$ at a scale of $0^{\prime} .187 \mathrm{pixel}^{-1}$.

(A color version of this figure is available in the online journal.)

\section{THE NGVS: DESCRIPTION OF THE SURVEY}

In 2007, CFHT issued a call for Large Programs that could be carried out over the eight semester period beginning in 2009A and continuing until 2012B. Four large programs were eventually approved ${ }^{33}$ including the NGVS, our new optical survey of the Virgo cluster. ${ }^{34}$ In this section, we describe the instrument-MegaPrime/MegaCam—used for the NGVS imaging, the adopted survey area, choice of field placements, filters and exposure times, as well as the observing and data acquisition strategies. For details regarding the data processing, calibration, reduction pipeline, and data products, see Section 4 (J.-C. Cuillandre et al. 2012, in preparation and S. D. J. Gwyn et al. 2012, in preparation). The scientific goals of the survey, which offers obvious synergies with the multi-wavelength studies discussed above, are described in detail in Section 5.

\subsection{CFHT and MegaPrime/MegaCam}

The instrument at the heart of the NGVS is MegaCam (Boulade et al. 2003), a wide-field optical imager mounted on MegaPrime, the newest prime focus of the $3.6 \mathrm{~m}$ CFHT. At the time of its first light in 2003, MegaCam was the largest imager ever built for astronomical observations and remains unrivaled at the time of the NGVS execution (2009-2013) for the combination of field of view, image quality, and depth of observations. The prime focus environment, MegaPrime, includes the following key components: (1) a wide-field optical corrector delivering a remarkably uniform image quality over the entire square degree field of view, (2) an image stabilizing

\footnotetext{
$\overline{33}$ http://www.cfht.hawaii.edu/en/science/LP_08_12/index.php

$34 \mathrm{https} / / /$ www.astrosci.ca/NGVS/Home.html
}

unit effectively removing telescope wind shake, and (3) a focus stage assembly.

MegaCam consists of 36 back-illuminated CCDs with good sensitivity in the near-UV, fabricated by e2v Technologies, and arranged in a pattern of four squares of nine detectors ${ }^{35}$ (see Figure 3). Individual CCDs measure $2048 \times 4612$ pixels, each of size $13.5 \mu \mathrm{m}$ and projecting to $0{ }^{\prime} .187$ on the sky; the typical read noise is $\approx 5 e \mathrm{pixel}^{-1}$. Each 340 megapixel image therefore covers an area of $0.96 \times 0.94 \simeq 0.90 \mathrm{deg}^{2}$. The cosmetic quality of the mosaic is excellent, with only $\sim 0.2 \%$ of the camera pixels being unresponsive. The instrument includes a filter jukebox that can accommodate as many as eight $30 \mathrm{~cm}^{2}$ filters that are moved into the field of view by a loading arm. Filter change and detector readout times are $120 \mathrm{~s}$ and $40 \mathrm{~s}$, respectively, with two amplifiers used to read out each CCD.

MegaCam observations are carried out exclusively via Queue Service Observing (QSO) at CFHT. The implementation of NGVS within the QSO framework is discussed in Section 4.

\subsection{Survey Geometry}

The pioneering study of Binggeli et al. (1985) surveyed an irregularly shaped region covering $\approx 140 \mathrm{deg}^{2}$ centered on the core of the Virgo cluster. A total of 67 du Pont plates were used to map out the large-scale structure of the cluster, with the number and relative positions of the fields chosen (somewhat arbitrarily) to encompass both the main body of the cluster and its immediate surroundings, including the $\mathrm{M}, \mathrm{W}, \mathrm{W}^{\prime}$ clouds and the southern extension (see, e.g., Binggeli et al. 1993, and references therein). Binggeli et al. (1987), and many subsequent

\footnotetext{
35 Four spare CCDs, located in the two middle rows, are not meant to be used for science as they are mostly vignetted by the filter frame.
} 
Table 1

NGVS Survey Region and Virgo Subcluster Properties

\begin{tabular}{|c|c|c|c|c|c|c|c|c|c|c|c|c|}
\hline \multirow[t]{2}{*}{ Subcluster } & \multirow[t]{2}{*}{ BCG } & \multirow{2}{*}{$\begin{array}{c}\alpha(\mathrm{J} 2000) \\
(\mathrm{h}: \mathrm{m}: \mathrm{s})\end{array}$} & \multirow{2}{*}{$\begin{array}{c}\delta(\mathrm{J} 2000) \\
(\circ: /: / \prime)\end{array}$} & \multirow{2}{*}{$\begin{array}{c}l \\
(\mathrm{deg})\end{array}$} & \multirow{2}{*}{$\begin{array}{c}b \\
\text { (deg) }\end{array}$} & \multirow{2}{*}{$\begin{array}{c}\lambda \\
(\operatorname{deg})\end{array}$} & \multirow{2}{*}{$\begin{array}{c}\beta \\
(\mathrm{deg})\end{array}$} & \multirow{2}{*}{$\begin{array}{c}\text { Mass } \\
\left(10^{14} \mathcal{M}_{\odot}\right)\end{array}$} & \multicolumn{2}{|c|}{$R_{200}$} & \multicolumn{2}{|c|}{$\Delta R / R_{200}$} \\
\hline & & & & & & & & & (deg) & $(\mathrm{Mpc})$ & (A) & (B) \\
\hline A & M87 & $12: 30: 49.4$ & $+12: 23: 28$ & 283.778 & 74.491 & 182.059 & 14.417 & 4.2 & 5.38 & 1.55 & $\ldots$ & $\cdots$ \\
\hline B & M49 & $12: 29: 46.7$ & $+08: 00: 02$ & 286.922 & 70.196 & 183.633 & 10.296 & 1.0 & 3.33 & 0.96 & $\ldots$ & $\ldots$ \\
\hline B1 & $\ldots$ & $13: 28: 25.5$ & $+21: 01: 04$ & 0.000 & 79.584 & 191.734 & 27.956 & $\cdots$ & $\ldots$ & $\ldots$ & 3.02 & 5.77 \\
\hline B2 & $\cdots$ & $12: 04: 34.3$ & $+27: 21: 19$ & 209.000 & 79.584 & 169.379 & 25.381 & $\ldots$ & $\cdots$ & $\cdots$ & 3.01 & 6.07 \\
\hline B3 & $\ldots$ & $11: 27: 03.6$ & $+15: 39: 08$ & 239.000 & 67.306 & 166.212 & 11.109 & $\ldots$ & $\ldots$ & $\ldots$ & 2.94 & 5.14 \\
\hline B4 & $\cdots$ & $13: 33: 34.2$ & $+06: 45: 34$ & 331.000 & 67.306 & 199.061 & 15.356 & $\cdots$ & $\cdots$ & $\cdots$ & 3.06 & 4.58 \\
\hline
\end{tabular}

studies, have amply demonstrated that not only is the cluster itself quite structurally complex, with a clumpy distribution that is concentrated around two main substructures, of which the larger has a somewhat elongated distribution on the sky (e.g., West \& Blakeslee 2000; Mei et al. 2007), but that this structure is itself embedded within an extended network of filaments and low-mass groupings (Tully \& Fisher 1987; Klypin et al. 2003). Thus, the question of the precise "boundaries" of the cluster requires careful consideration when planning a new modern survey.

In deciding upon the NGVS survey region, we are able to build upon the large amount of X-ray and radial velocity data that is now available for the Virgo cluster. As shown by Binggeli et al. (1987), the cluster is dominated by two major components $^{36}$ (see Table 1). The main concentration, termed subcluster A, is centered on M87 (VCC1316) and characterized by a high-velocity dispersion of $\sigma_{v} \sim 700-800 \mathrm{~km} \mathrm{~s}^{-2}$. Roughly $4.4(\sim 1.25 \mathrm{Mpc})$ to the south, the smaller B subcluster with $\sigma_{v} \sim$ $400-500 \mathrm{~km} \mathrm{~s}^{-2}$ is centered on M49 (VCC1226), the optically brightest member of the Virgo cluster. Extensive X-ray data are available for both of these subclusters, along with radial velocity measurements for hundreds of galaxies (and GCs surrounding the central galaxies), so it is possible to combine these data selfconsistently to infer the distribution of gravitating mass within each subcluster.

Such an analysis was performed by McLaughlin (1999), who combined surface photometry for M87, radial velocity measurements for early-type galaxies, and ROSAT X-ray observations from Nulsen \& Böhringer (1995) to construct a mass model for the Virgo A subcluster. For an adopted distance of $16.5 \mathrm{Mpc}$ (see Mei et al. 2007), the basic parameters of this mass model are

$$
\begin{aligned}
a(M 87) & =70^{\prime \prime} 13 \\
R_{s}(\mathrm{DM}) & =110 a \simeq 2.143(=0.617 \mathrm{Mpc}) \\
R_{200}(\mathrm{DM}) & =2.51 R_{s} \simeq 5.383(=1.55 \mathrm{Mpc}) \\
\mathcal{M}_{200}(\mathrm{DM}) & =4.2 \times 10^{14} \mathcal{M}_{\odot},
\end{aligned}
$$

where $a(M 87)$ is the scale radius of the Dehnen (1993) model fitted to the M87 surface brightness profile and $R_{S}(\mathrm{DM})$ is the scale radius of the NFW (Navarro et al. 1997) model fitted to the surrounding DM halo. As is customary, the virial radius is taken to be $R_{v} \approx R_{200}(\mathrm{CLDM}) \equiv c R_{s}(\mathrm{DM})$, where $c$ is the DM halo concentration parameter. The mass enclosed within this radius is denoted by $\mathcal{M}_{200}(\mathrm{DM})$.

\footnotetext{
36 Additional components, such as those associated with M86 (VCC881) or M60 (VCC1978), are much less massive than the A and B subclusters (see, e.g., Binggeli 1999; Schindler et al. 1999).
}

Applying this same methodology to M49 and the Virgo B subcluster, we find

$$
\begin{aligned}
a(\mathrm{M} 49) & =38^{\prime \prime} .78 \\
R_{s}(\mathrm{DM}) & =69.5 a \simeq 0.749(=0.215 \mathrm{Mpc}) \\
R_{200}(\mathrm{DM}) & =2.51 R_{s} \simeq 3.334(=0.96 \mathrm{Mpc}) \\
\mathcal{M}_{200}(\mathrm{DM}) & =1.0 \times 10^{14} \mathcal{M}_{\odot} .
\end{aligned}
$$

This indicates that $\approx 80 \%$ of the total cluster mass is contained within the A subcluster. The virial radii of the A and B subclusters calculated in this way are indicated by the red circles in Figure 1. The centers of the two subclusters are shown by large red crosses, which mark the location of M87 and M49, respectively. The area inside the solid red curves comprises the NGVS survey region, which therefore provides complete coverage of the two subclusters out to their virial radii. We note that Urban et al. (2011) have recently argued for a rather more compact and less massive cluster than indicated above, based on X-ray spectroscopy of the Virgo A subcluster using multiple overlapping XMM-Newton pointings extending 4.5 northward from M87. Combining the mean temperature of $k T=2.3 \mathrm{keV}$ measured by Shibata et al. (2001) from ASCA observations covering an area of $19 \mathrm{deg}^{2}$ with the cluster scaling relations of Arnaud et al. (2005), they found $R_{200} \approx 1.08 \mathrm{Mpc}$ (3.75 for our adopted distance of $16.5 \mathrm{Mpc}$ ) and $\mathcal{M}_{200} \approx 1.4 \times 10^{14} \mathcal{M}_{\odot}$. If one adopts these parameters for Virgo, then the NGVS fields provide full coverage out to $1.5 R_{200}$ in the A subcluster.

The NGVS survey area $\left(\approx 104 \mathrm{deg}^{2}\right)$ is $\sim 75 \%$ of that of the VCC (Binggeli et al. 1987). Most of the "missing" coverage is at low declination $\left(\delta_{2000} \lesssim 5^{\circ}\right)$, which is dominated by the southern extension, a distinct structure defining a filamentary "spur" that extends from Virgo toward the background (Tully 1982; Binggeli et al. 1993; Hoffman et al. 1995). North of M49 $\left(\delta_{2000} \approx 8^{\circ}\right)$, all but 55 of the confirmed or probable Virgo members from the VCC $(96 \%)$ are contained within the NGVS survey region, which also includes a $\sim 4 \mathrm{deg}^{2}$ area outside of the VCC coverage (mostly in the northeast quadrant).

Figure 4 shows the placement of the MegaCam fields for the NGVS. As described above, the virial radii of the A and B subclusters define the outer boundaries of the NGVS, and are again shown by the red outline. Individual MegaCam pointings are shown in gray. The fields are labeled in blue, using a naming convention that gives the offset, in right ascension and declination and to the nearest degree, from the $(0,0)$ field which includes, in its northwest corner, M87. Based on this convention, M49 is located in field $(-1,-4)$. As a consequence of the dithering strategy described in Section 3.5, each NGVS field projects to exactly $1^{\circ} \times 1^{\circ}$ on the plane of the sky. By design, adjacent fields overlap by $3^{\prime}$ at the edges. The overlap region serves multiple purposes: (1) it allows us to test the photometric consistency between frames (although the main photometric 


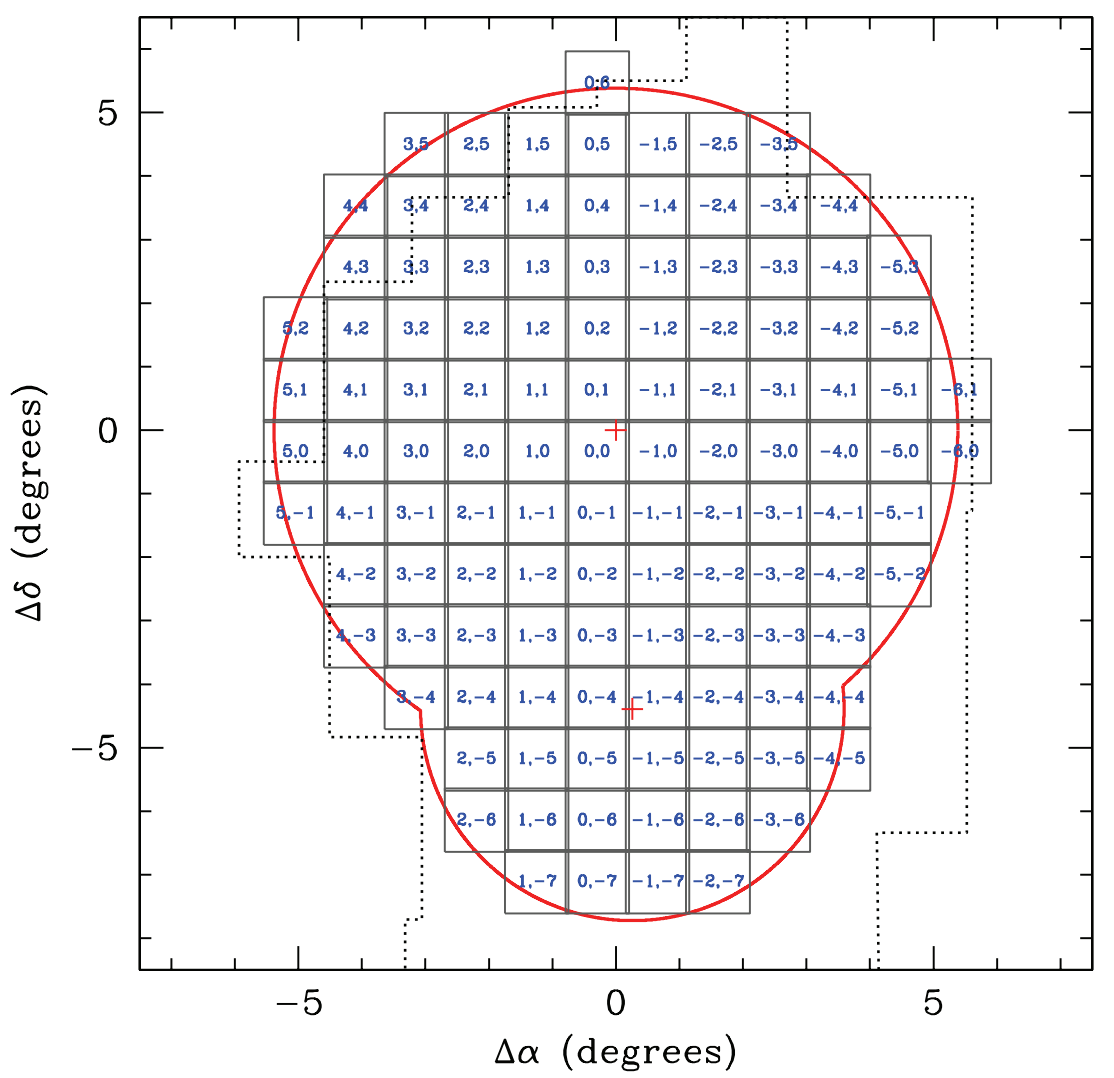

Figure 4. Placement of MegaCam fields for the NGVS (gray squares). The red curves show the virial radii for the A and B subclusters (see Figure 1), while the dotted black lines show the VCC region surveyed by Binggeli et al. (1985). Individual NGVS fields are labeled in blue. The two red crosses identify the location of M87 (to the north) and M49 (to the south), located at the center of the A and B subclusters, respectively.

(A color version of this figure is available in the online journal.)

calibration is performed against SDSS images, see Section 4.2); (2) it provides a check for astrometric accuracy when joining multiple fields; (3) it allows us to recover the signal-to-noise ratio $(\mathrm{S} / \mathrm{N})$ at the field edges, which are necessarily less well sampled than the central parts by the dithering procedure; and (4) it allows us to test the effectiveness of our data processing pipeline (Elixir- $L S B$, see Section 4.1) in recovering the true sky background by comparing possible differential gradients between adjacent fields. Because of the overlap between fields, a total of 117 pointings are necessary to cover the $104 \mathrm{deg}^{2}$ survey area.

Figure 5 shows the NGVS outline superimposed on a Galactic extinction map from Schlegel et al. (1998). Also shown are four "background" fields. These are located three virial radii $(\approx 16 \mathrm{deg})$ from the cluster center, and at Galactic latitudes matching those of the lower and upper boundaries of the NGVS fields. The background fields are observed with the same filters, exposure times, and dithering strategy adopted for the NGVS Virgo fields, and provide a statistical estimate of the contamination to the NGVS fields due to background galaxies and foreground (Galactic) stars. The background fields are representative of the near-Virgo background environment, and none project onto regions believed to be infalling onto the cluster (such as the southern extension mentioned above). Specifically, the northeast background field is located in as empty a part of the sky as one can find in the Virgo region. The northwest field is near Coma I, which is roughly at the Virgo distance but has a large spread in systemic velocity. The southwest field projects near the UMa Southern Spur, which has systemic velocity between 1500 and $2500 \mathrm{~km} \mathrm{~s}^{-1}$ and is about twice as far as Virgo $(\sim 20-30 \mathrm{Mpc})$. Finally, the southeast background field projects on the Virgo-Libra cloud, which is located at $\sim 25 \mathrm{Mpc}$ and has systemic velocity of $\sim 2000 \mathrm{~km} \mathrm{~s}^{-1}$. Coma I, the UMa Southern Spur, and the Virgo-Libra cloud contain between one to two dozen known members.

\subsection{Choice of Filters}

MegaCam is equipped with $u^{*}, g, r, i, z$ filters, ${ }^{37}$ the transmission functions of which are shown in Figure 6, including the response of the primary mirror, the optics, and the quantum efficiency of the CCDs. The filters are similar, but not identical, to standard SDSS filters. The most significant difference is for the $u^{*}$ filter, which was designed to have a redder and broader response than SDSS $u$ to take advantage of the improved near-UV capabilities of CFHT on Mauna Kea. Fringing in the raw data is at the $\sim 6 \%$ level in the $i$ band, and $\sim 15 \%$ in $z$. However, the detrending recipe implemented in the pipeline combo for the NGVS data, Elixir plus Elixir-LSB (Section 4.1), virtually eliminates fringing in $i$ and reduces it to $<1 \%$ in $z$.

Although the original plan called for the entire NGVS survey area to be covered in all five filters, at the time of writing, it appears likely that the $r$-band coverage and or exposure times will need to be somewhat reduced (see Section 4.4). As discussed in

\footnotetext{
37 Several designations are used to denote MegaCam filters. For simplicity, we adopt $u^{*}, g, r, i, z$, since of all filters only $u^{*}$ is significantly different from SDSS $u$. All NGVS observations use the "new" i.MP9702 $i$-band filter which was installed in 2007 October.
} 


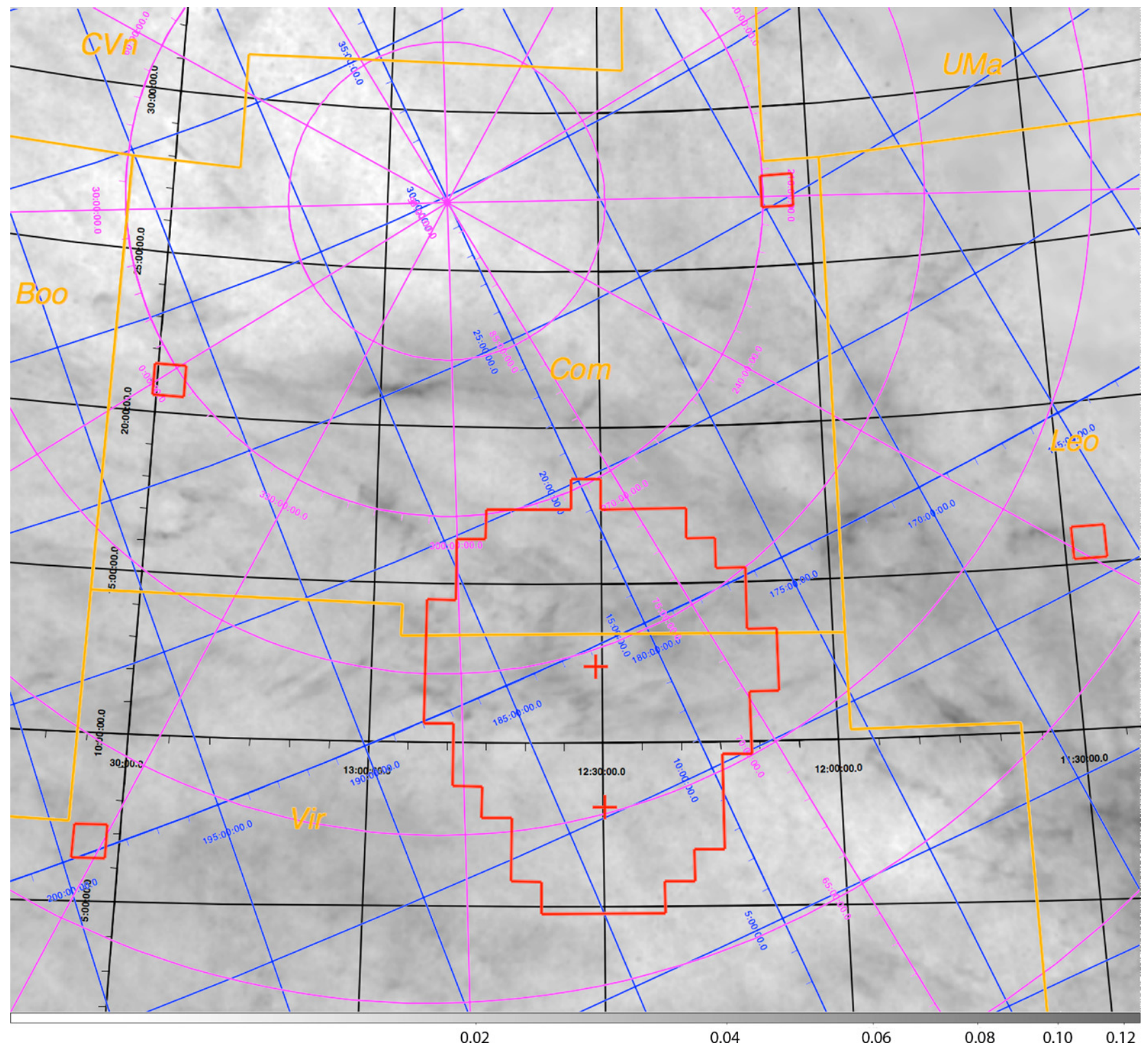

Figure 5. Region surveyed by the NGVS overlaid on the Galactic extinction map from Schlegel et al. (1998). The gray scale corresponds to $E(B-V)$ values, as shown in the legend at the bottom. The area in red shows the footprint of the NGVS, which covers an area of $104 \mathrm{deg}^{2}$, as well as four "background" fields ( 4 deg' in total) that are located three virial radii $(\approx 16.5 \mathrm{deg})$ from the cluster center. The crosses indicate the location of M87 and M49, which mark the centers of Virgo's two main substructures. Blue and magenta lines show grids in ecliptic and Galactic coordinates, respectively. The NGVS-the largest contiguous field mapped to this depth at optical wavelengths $(g=25.9 \mathrm{mag}, 10 \sigma)$ —defines a low-extinction sight line at relatively high Galactic latitude $\left(\langle b\rangle \sim 74^{\circ}\right)$ and low ecliptic latitude $\left(\langle\beta\rangle \sim 14^{\circ}\right)$.

Section 5, a panchromatic view is needed to achieve the science goals of the NGVS: In particular, the long wavelength baseline $\left(u^{*}\right.$ to $\left.z\right)$ is essential to decouple age and metallicity effects when comparing the data against stellar population models, with $u^{*}$ playing a crucial role in revealing recent or ongoing star formation. Moreover, $u^{*}$ is crucial for distinguishing background galaxies from cluster members and for a seamless comparison of Virgo's luminosity function, star formation properties, and color-magnitude diagrams (CMDs) to those of more distant clusters. Similarly, stellar mass measurements hinge critically on the redder passbands, $z$ in particular. All five filters are needed to measure the photometric redshifts (which are critical in assessing background contamination), perform stellar population work, and study high-redshift galaxy clusters and cosmic shear (Section 5.3).

\subsection{Observing Requirements, Exposure Times, and Limiting Magnitudes}

Exposure times and observing constraints are listed in Table 2 for each filter. The main data set consists of long exposures, with total integration per field ranging from $0.57 \mathrm{hr}$ (in $i$ ) to $1.78 \mathrm{hr}$ (in $u^{*}$ ). The limiting magnitudes and surface brightnesses corresponding to these exposure times are listed in Table 2 for a fiducial airmass of 1.2 and for the maximum seeing FWHM admissible for the survey (listed in Column 7). Given these limiting magnitudes, NGVS images yield a census of baryonic substructures in Virgo to a completeness limit similar to what is currently achieved in the Local Group: In $g, r$, and $i$, all but the very faintest of the Milky Way and M31 (i.e., And I to $\mathrm{XV}$ ) satellites would be detected with an integrated $\mathrm{S} / \mathrm{N} \geqslant 2$ 


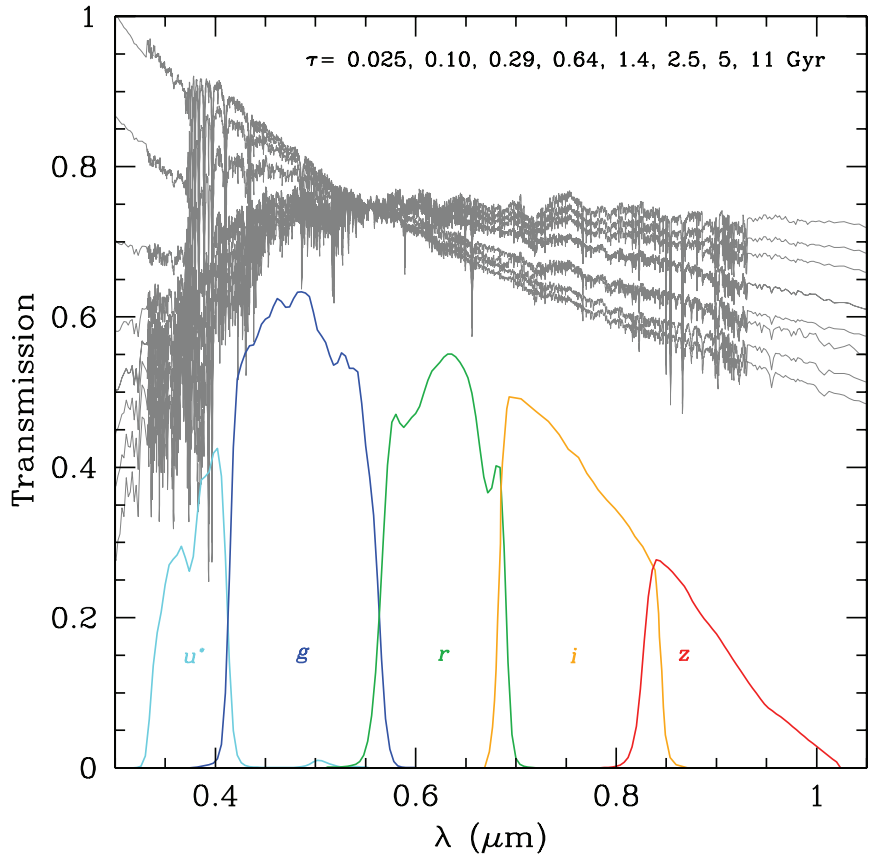

Figure 6. Total transmission curves for the CFHT/MegaPrime $u^{*}$ griz filter set used in the NGVS. Note that these curves show the combined transmission for mirrors, optics, and detectors. For reference, the spectra plotted in gray show SSP models from Bruzual \& Charlot (2003), with solar metallicity and ages of $t=0.025,0.10,0.29,0.64,1.4,2.5,5$, and $11 \mathrm{Gyr}$.

(A color version of this figure is available in the online journal.)

within one effective radius if they were moved to the distance of Virgo. For compact and unresolved sources, such as GCs and "ultracompact dwarf" (UCD) galaxies, the same exposure times allow the detection (at $S / N \geqslant 10$ ) of the brightest $90 \%$ of the luminosity function, which has a roughly Gaussian form with peak magnitude $g \simeq 24.0$ and dispersion $\sigma=1.3 \mathrm{mag}$ (Jordán et al. 2007). In $u^{*}$ and $z$, due to the lower detector efficiency, the latter $\mathrm{S} / \mathrm{N}$ requirement is relaxed to $\mathrm{S} / \mathrm{N}=5$; furthermore, since the $u^{*}$ band is mostly needed for young stellar population studies, its depth is relaxed by a further $0.6 \mathrm{mag}$, from the $u^{*}=$ 26.9 mag appropriate for an old, metal-poor stellar population to $u^{*}=26.3 \mathrm{mag}$. The $\mathrm{S} / \mathrm{N}$ achieved in the long exposures is shown in Figure 7 as a function of magnitude, in each filter, assuming unresolved sources. Throughout this paper, the $\mathrm{S} / \mathrm{N}$ is calculated in an optimal aperture of radius equal to two-third the seeing FWHM. However, in Table 2 we used the upper bound of the admissible FWHM for each filter, while in Figure 7 we used the (lower) median FWHM actually measured for the NGVS exposures (as shown in Figure 8). For this reason, the $\mathrm{S} / \mathrm{N}$ shown in Figure 7 is slightly higher than reported in Table 2.

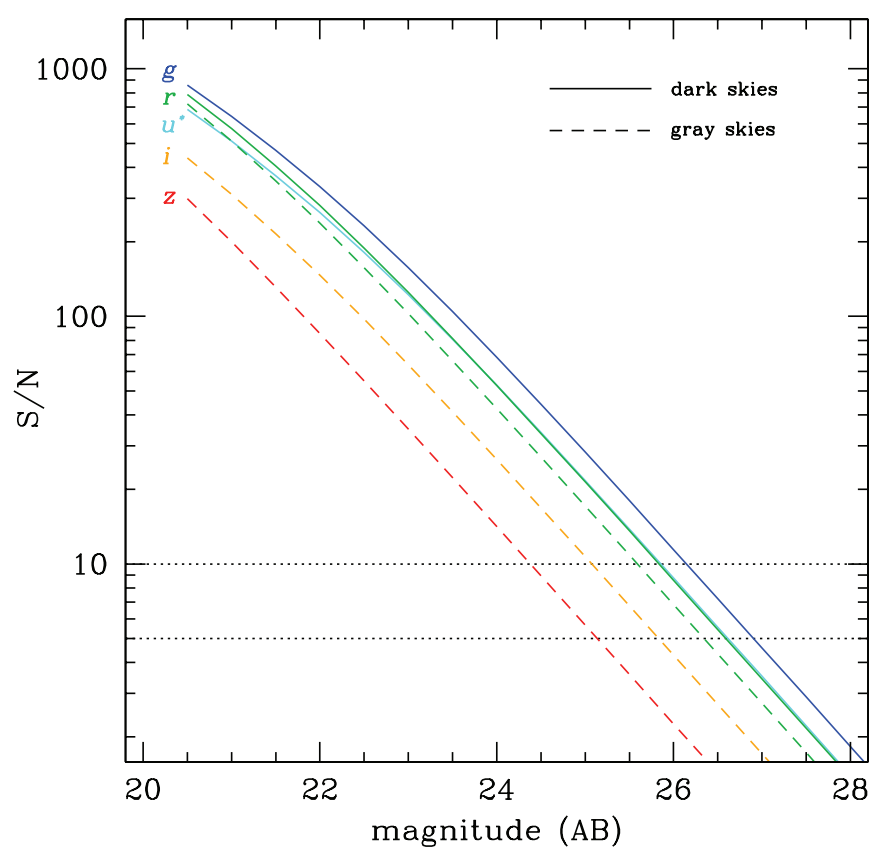

Figure 7. Point-source signal-to-noise ratio $(\mathrm{S} / \mathrm{N})$ as a function of magnitude at the full depth of the stacked NGVS images. Curves show results obtained in the different bandpasses. Dark sky conditions are assumed for $u^{*}$ and $g$; for the $r$ band we show the $\mathrm{S} / \mathrm{N}$ expected under dark and gray sky conditions as solid and dashed curves, respectively. For the $i$ and $z$ filters, there is essentially no difference between dark and gray sky conditions, although it should be noted that the sky background in those bands varies significantly with airmass, a parameter that is loosely constrained for the NGVS. All calculations assume an airmass of 1.2 and seeing equal to the median value measured in each filter from all NGVS data obtained between 2009 and 2011 (as shown in Figure 8), The $\mathrm{S} / \mathrm{N}$ is calculated in an optimal aperture with radius equal to two-thirds of the measured FWHM for point sources. Dotted lines are drawn at $\mathrm{S} / \mathrm{N}=5$ and 10: Note that the limiting magnitudes shown in this figure do not necessarily correspond to those listed in Table 2, which were calculated assuming the worse acceptable seeing for the NGVS exposures (1". 1 in $u^{*}, 1^{\prime \prime} .0$ in $g, r, z$, and $0^{\prime \prime} 6$ in $i)$.

(A color version of this figure is available in the online journal.)

Note that all NGVS $u^{*}$ and $g$ data are required to be taken under dark sky conditions (Moon illumination $\leqslant 10 \%$ ), while gray skies (Moon illumination $\leqslant 40 \%$ ) are acceptable in $r$, and all conditions are acceptable in $i$ and $z$.

All long exposures are divided into a number of dithered sub-exposures to allow removal of cosmic rays and sampling of the intra-chip gaps within the MegaCam mosaic (Section 3.5). However, even in single exposures, the centers of most bright galaxies are expected to be saturated. For this reason, short exposures are also obtained for each field. The total integration time of the short exposures (between $40 \mathrm{~s}$ and $250 \mathrm{~s}$ ) is chosen

Table 2

NGVS Exposure Breakdown Per Field and Queue Service Observing Criteria

\begin{tabular}{|c|c|c|c|c|c|c|c|}
\hline \multirow[t]{2}{*}{ Filter } & \multicolumn{3}{|c|}{ Long Exposures } & \multicolumn{2}{|c|}{ Short Exposures } & \multirow{2}{*}{$\begin{array}{l}\text { Seeing } \\
\text { FWHM } \\
(\operatorname{arcsec})\end{array}$} & \multirow{2}{*}{$\begin{array}{c}\text { Moon } \\
\text { Illumination } \\
(\%)\end{array}$} \\
\hline & $\begin{array}{l}\text { Exp. Time } \\
\text { (s) }\end{array}$ & $\begin{array}{l}\text { Point-source Limit } \\
\text { (mag) }\end{array}$ & $\begin{array}{l}\text { Extended-source Limit } \\
\quad\left(\operatorname{mag} \operatorname{arcsec}^{-2}\right)\end{array}$ & $\begin{array}{l}\text { Exp. Time } \\
\text { (s) }\end{array}$ & $\begin{array}{l}\text { Point-source Limit }{ }^{\mathrm{a}} \\
\text { (mag) }\end{array}$ & & \\
\hline$u^{*}$ & $11 \times 582$ & $26.3(\mathrm{~S} / \mathrm{N}=5)$ & 29.3 & $5 \times 50$ & 24.3 & $\leqslant 1^{\prime \prime} 1$ & $\leqslant 10 \%$ \\
\hline$g$ & $5 \times 634$ & $25.9(\mathrm{~S} / \mathrm{N}=10)$ & 29.0 & $5 \times 12$ & 23.2 & $\leqslant 1^{\prime \prime} 0$ & $\leqslant 10 \%$ \\
\hline$r$ & $7 \times 687$ & $25.3(\mathrm{~S} / \mathrm{N}=10)$ & 27.2 & $5 \times 9$ & 22.2 & $\leqslant 1^{\prime \prime} .0$ & $\leqslant 40 \%$ \\
\hline$i$ & $5 \times 411$ & $25.1(\mathrm{~S} / \mathrm{N}=10)$ & 27.4 & $5 \times 8$ & 21.7 & $\leqslant 0^{\prime \prime} 6$ & Any \\
\hline$z$ & $8 \times 550$ & $24.8(\mathrm{~S} / \mathrm{N}=5)$ & 26.0 & $5 \times 13$ & 21.2 & $\leqslant 1^{\prime \prime} 0$ & Any \\
\hline
\end{tabular}

Note. ${ }^{\text {a }}$ The $\mathrm{S} / \mathrm{N}$ is calculated in an optimal aperture of size equal to two-third the seeing FWHM, listed in Column 7, and assumes an airmass of 1.2. 


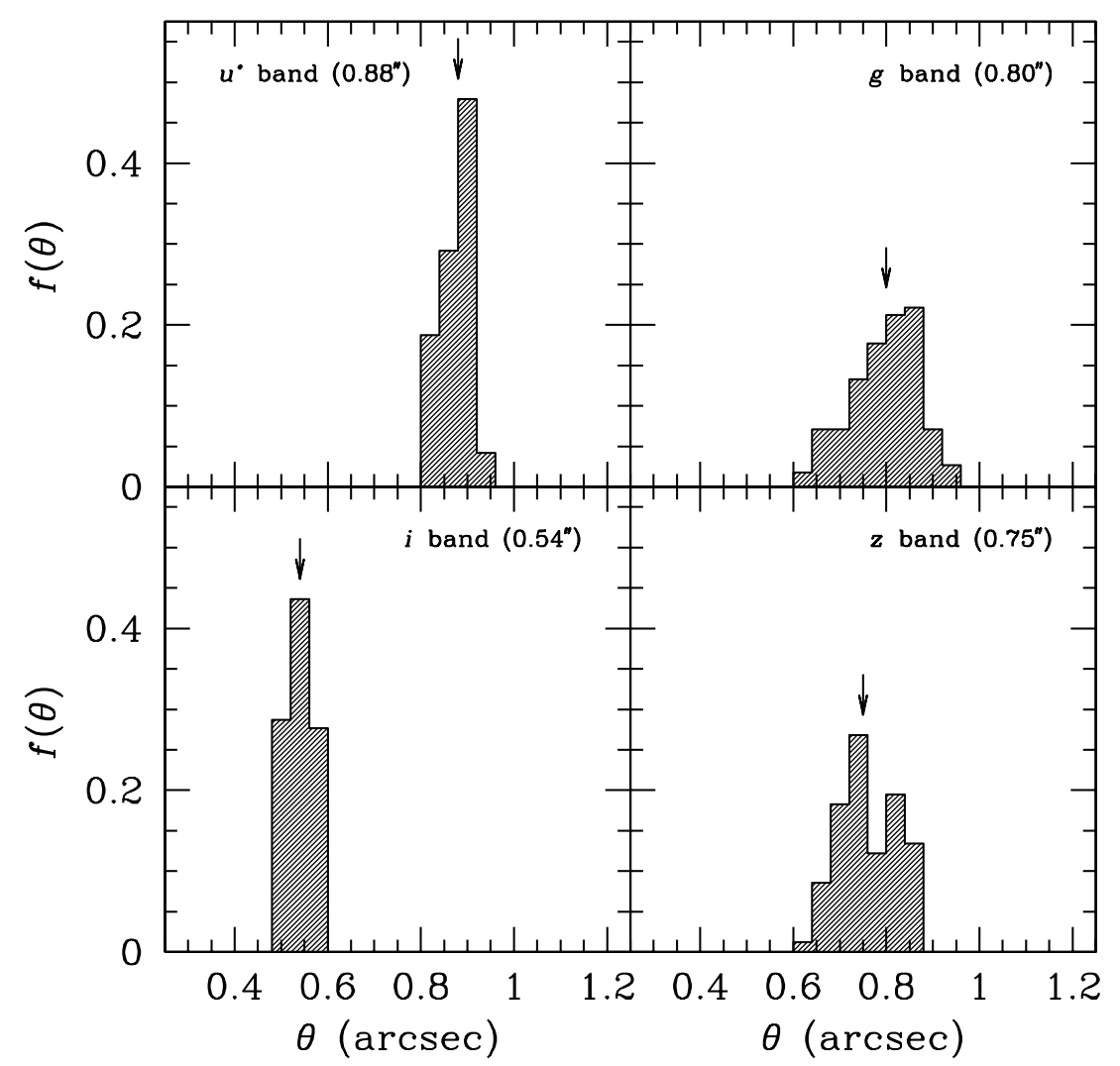

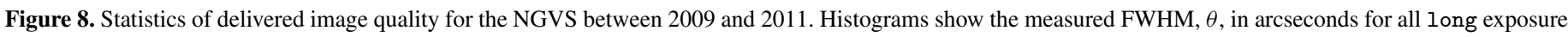

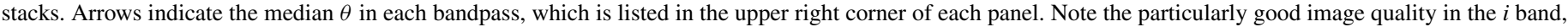
where $\theta \lesssim 0^{\prime \prime} 6$ is required for validated exposures.

to ensure that the centers of all Virgo galaxies are observed in the linear regime. ${ }^{38}$ Short exposures are generally readnoise-dominated and are therefore never combined to the long exposures.

NGVS data are acquired under strict observing conditions. The seeing FWHM, defined as the average value across the entire mosaic, cannot exceed $1^{\prime \prime}$ in $g, r$, and $z$. To increase the window of opportunity for observations in $u^{*}$, which require both dark skies and long integration times, seeing constraints were relaxed to $1^{\prime \prime} .1$ in this band. Exceptional seeing is desirable in at least one band for the study of the nuclear structure of Virgo galaxies, compact stellar systems (including GCs), and the measurement of surface brightness fluctuation (SBF) distances (see Sections 5.1.4, 5.1.5, 5.1.6, and 5.1.7). The natural choice for this is the $i$ band, for which (with $z$ ) the best seeing is routinely recorded at CFHT, and the high CCD efficiency translates into relatively short exposure times, thus lessening the scheduling constraints (whereas $z$, besides being more severely affected by fringing, also requires longer integrations). Seeing for all $i$-band NGVS images is required not to exceed $0{ }^{\prime \prime} 6$. In practice, these requirements do not set unreasonable constraints on the queue observing, since the median $r$-band seeing on MegaCam data is 0!'7 (J.-C. Cuillandre \& F. Y. C. Racine 2011, private communication). Figure 8 shows seeing statistics for all NGVS data obtained as of 2011 September (i.e., for the first three years of operations; note that no $r$-band data are available yet, see Section 4.4). The median seeing, defined as the average FWHM for point sources across the entire mosaic, is indicated

\footnotetext{
38 MegaCam's response is linear over the entire dynamic range of the 16 bits encoded data.
}

in each panel. Since the NGVS is a high-priority program within the QSO, it naturally samples better conditions than requested to conduct the science goals. As a result, in all bands the data were obtained in significantly better seeing than required, in particular all $z$ images have seeing $<0$ '.9 (median seeing 0.75), and all $u^{*}$ data have seeing $<1^{\prime \prime}$.0 (median seeing 0".88).

Although most NGVS data are obtained under photometric conditions, this is not a strict a priori requirement, since the entire NGVS survey area is covered by the SDSS, which in itself provides a photometric reference frame for our survey (Section 4.2). However, no data are taken if the extinction exceeds 0.1 mag. ${ }^{39}$ Furthermore, if conditions are nonphotometric, we require the Moon illumination to be less than $10 \%$ and the Moon to be at least $40^{\circ}$ away from the NGVS fields. These constraints ensure a stable sky background between exposures, a condition necessary for the implementation of the Elixir- $L S B$ processing pipeline described in Section 4.2. For the same reasons, no data are obtained when the airmass exceeds 1.5 , or in more than $12^{\circ}$ twilight.

The observing constraints described above lead to an exceptionally homogeneous data set. They do, however, mean that the program cannot be executed in classical mode, as it requires very efficient queue schedule observing, such as the one in operation at CFHT.

\subsection{Data Acquisition and Observing Strategy}

Adjacent CCDs in the MegaCam mosaic are separated by physical gaps, giving a $93 \%$ filling factor for the entire mosaic.

\footnotetext{
39 The extinction is measured in real time on the $V$-band science field of the SkyProbe system mounted on CFHT (Cuillandre et al. 2002).
} 


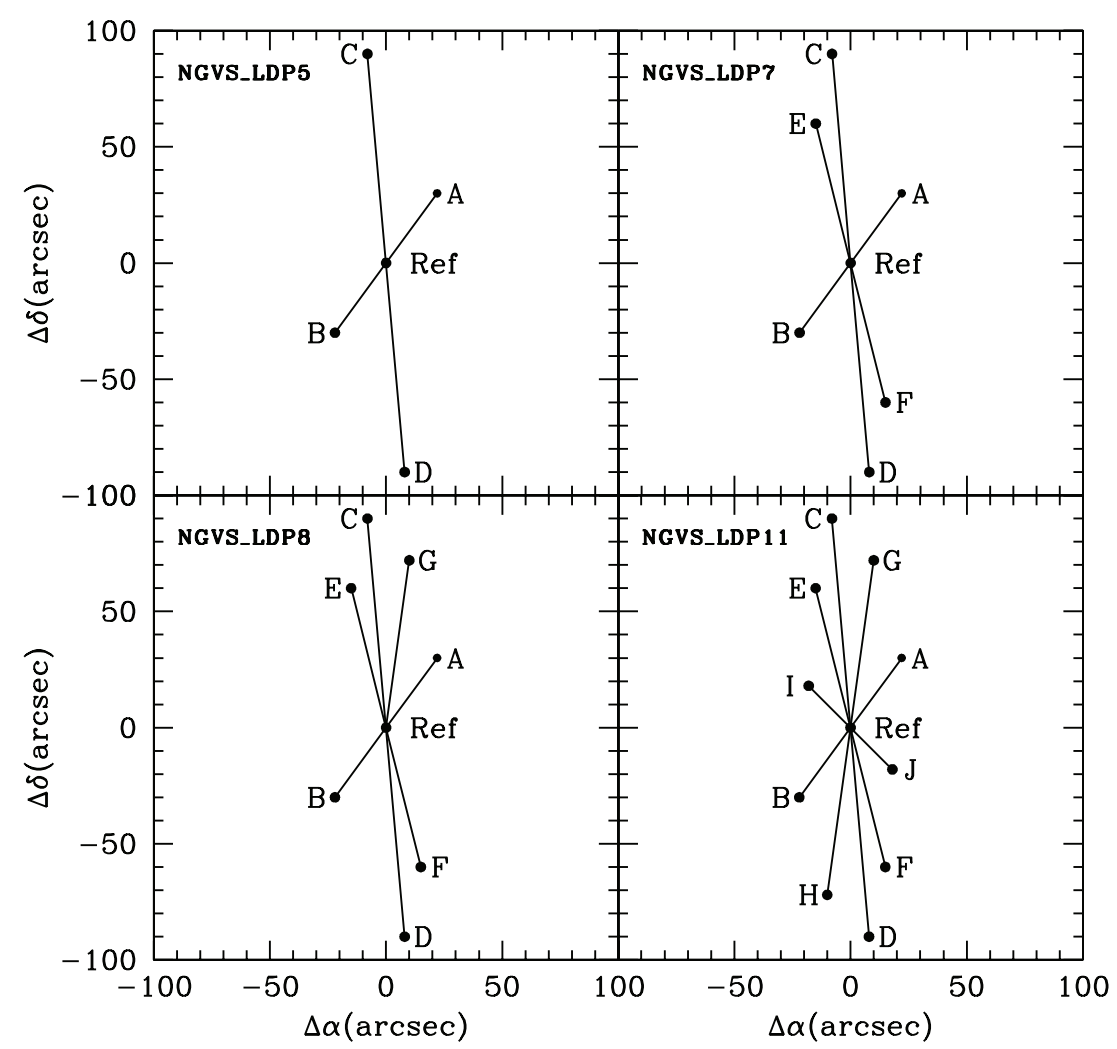

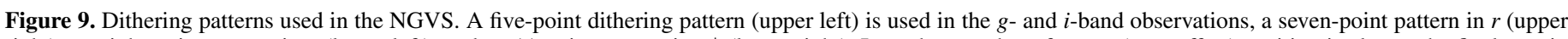

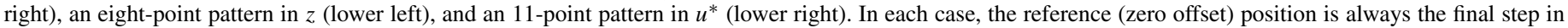
the dithering sequence.

The gaps project on the sky to strips with width varying between $\sim 6^{\prime \prime}$ and $\sim 14^{\prime \prime}$, except for the gaps between the first and second, and third and fourth rows of CCDs, which are separated by $\sim 73^{\prime \prime}$ to provide the necessary space for the CCDs' wire bonds and connectivity (Figure 3 ). To recover the intra-chip regions, NGVS images are dithered following the standard MegaCam "large dither pattern," as shown in Figure 9. Dithered images are offset from the reference position by $8^{\prime \prime}-18^{\prime \prime}$ in right ascension and by $12^{\prime \prime}-30^{\prime \prime}$ in declination - the larger offsets in declination are necessary to cover the wider vertical gaps between CCD rows. The number of dithering positions must be at least five to adequately cover the intra-CCD regions. For the long NGVS exposures, the number of points in the dithering pattern depends on the total integration time: the shortest exposures ( $g$ and $i$ ) use a five-point dithering patter, $r$ uses seven, $z$ uses eight, and $u^{*}$, which requires the longest total integration, uses 11 . All short exposures are dithered according to a five-point dithering pattern, independent of the total integration. Proper resampling and stacking of the dithered frames (see Section 4.2) will then yield complete sky coverage over a $1 \mathrm{deg}^{2}$ area.

In a significant departure from the canonical data acquisition procedure, however, the NGVS does not acquire all exposures within a given dithering pattern as part of an uninterrupted sequence, as motivated below. Rather, a step-dither procedure is adopted: In each filter, a single exposure (corresponding to a given position in the dithering pattern) is acquired for each of a number (typically seven, but occasionally six) of separate fields before a second series of exposures (corresponding to the following position in the dithering patter) is obtained for the same fields. The sequence is repeated until all pointings in the dithering pattern are acquired for each field. In other words, the NGVS step-dithering procedure can be thought of as a dithering pattern applied to a group of fields, rather than to a single field. A schematic representation of an NGVS stepdither pattern is shown in Figure 10. The fields that are part of a group do not need to be contiguous in space (although they generally are, to minimize telescope slewing time). The only requirement for the procedure is that a minimum of seven consecutive exposures must be acquired in an uninterrupted (or nearly so, see below) sequence. However, there is no upper limit to the number of exposures that can be taken in a single sequence, nor on the time elapsed between successive sequences of $(\geqslant 7)$ exposures on the same field group in any given filter. It follows that for any given filter, images of the same field are often obtained days, weeks or, in extreme cases, even months apart. Because of the strict observing constraints required for the NGVS (Section 3.4), we found that this has no impact on the consistency of the image quality and photometric accuracy between exposures belonging to a single field.

The step-dither observing sequence described above allows us to push the surface brightness detection limit in the final NGVS images to levels that would otherwise be unattainable. The main limitation stems from contamination from light scattered in the focal plane by internal reflections in the Wide Field Corrector optics. In frames preprocessed using the standard CFHT pipeline (Elixir, Magnier \& Cuillandre 2004), scattered light contamination is further amplified by the use of twilight flat-field images, and is manifested as a circular pattern centered on the mosaic and spreading on scales of several CCDs, with a peak-to-peak variation in the number of counts between $\sim 15 \%$ and $\sim 25 \%$ of the sky, depending on the filter (larger in $u^{*}$ and $z$ ). Due to temporal variations in the night-time sky 


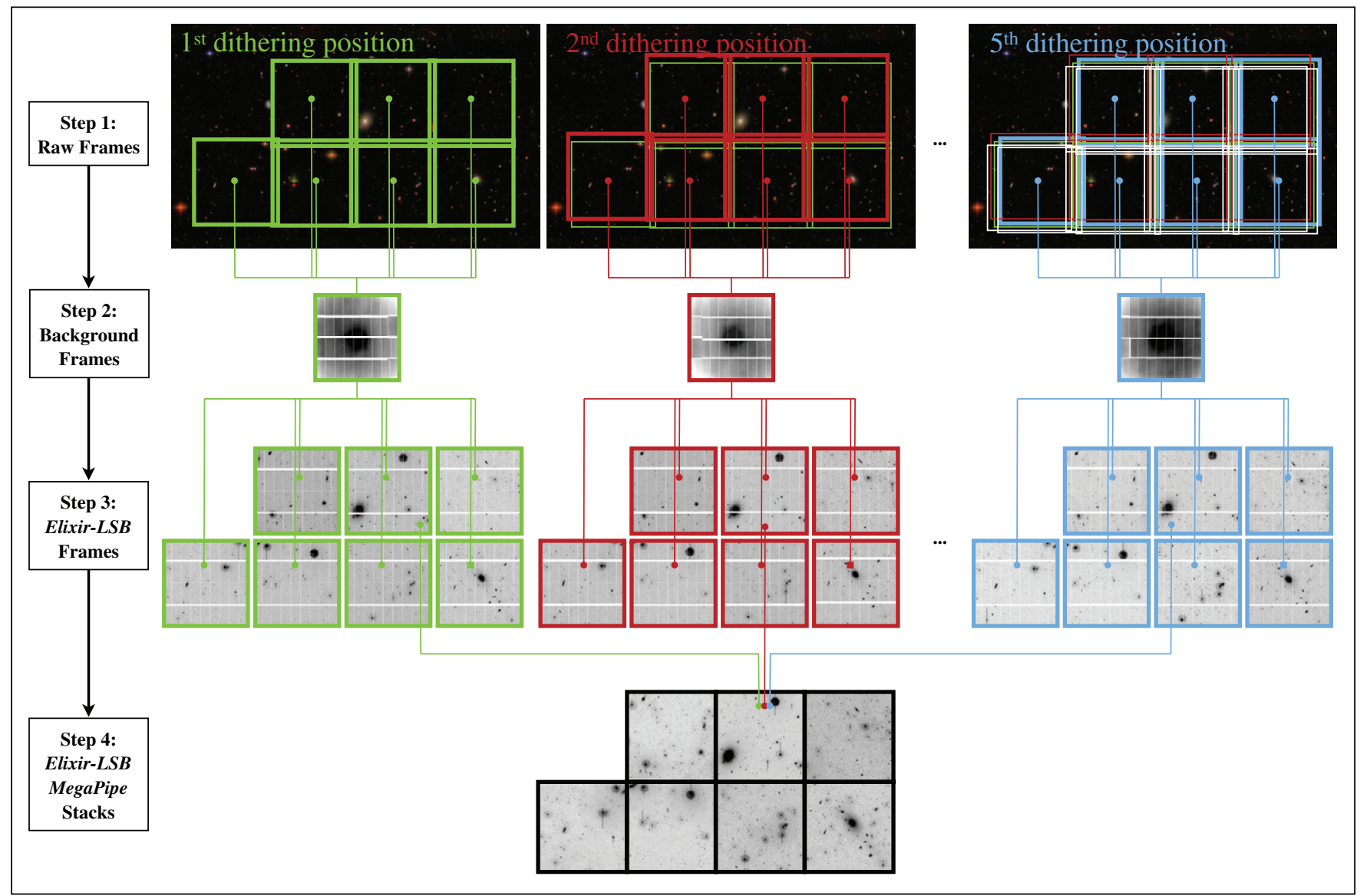

Figure 10. Schematic representation of the step-dither strategy adopted for the NGVS, which lies at the core of the Elixir-LSB observing strategy and processing pipeline. A single image is taken for each of seven $1 \mathrm{deg}^{2}$ fields, indicated in the top left panel by the green squares superimposed on an SDSS image of the M49 region ("step 1"; note that the fields overlap slightly). These seven images are acquired as an uninterrupted sequence, typically lasting less than 1 hr. Processing through Elixir-LSB combines these frames, rejecting real objects (stars, galaxies, etc.) and produces a single "scattered light" background image ("step 2"). This is then rescaled and subtracted from each of the seven images, producing flat, background-corrected Elixir-LSB frames ("step 3"). The entire sequence is then repeated (sometimes immediately after the first, but typically days or weeks later): The same seven fields are observed again, but this time at a slightly different offset position (corresponding to the second position in the dithering pattern adopted for that particular filter). These new images (identified by the red boxes in the top middle panel) produce a second background frame that is used to correct each exposure for scattered light. Once the last sequence of exposures is obtained (the figure assumes the case of a five-point dithering pattern, and dither positions 3 and 4 are not shown explicitly) and the corresponding frames are processed, all images belonging to the same field are stacked ("step 4"). See Sections 3.5, 4.1, and 4.2 for details.

(A color version of this figure is available in the online journal.)

brightness, the whole pattern changes in intensity at the several percent level on timescale of several hours, and cannot therefore be corrected by using a master flat field built from frames taken during the course of several nights.

Before the NGVS, the majority of the science investigated with MegaCam involved sources with small angular extent (e.g., point sources or high-redshift galaxies). As a consequence, early efforts on the Elixir pipeline were focused on compensating for the geometric distortion of the mosaic and delivering a photometrically flat image across the entire field of view. The large-scale, additive twilight flat-field signature due to scattered light was intentionally left in the data since it could be removed easily when combining dithered images. Spatially, scattered light varies appreciably on scales of several tens of arcseconds (the exact pattern depends on the filter). It follows that if the astrophysical objects under study have small spatial extent (less than a few tens of arcseconds, as is the case for stars or background galaxies), then scattered light can be corrected by subtracting a "local" estimate of the background when combining ("stacking") dithered exposures. This approach, however, fails if the objects of interest extend over larger scales, as is the case for all but the very faintest Virgo galaxies, or for the diffuse web of filaments and streams that permeate the intracluster medium.

An alternative approach for characterizing the scattered light component and natural sky brightness variations is to median combine exposures of different fields, if these are all taken within a period not exceeding the time frame over which the scatter light changes significantly (i.e., $\sim 1 \mathrm{hr}$ in the case of the NGVS). Given the typical level of crowding in the NGVS fields, at least six fields must be median-combined to ensure that the real objects are removed in the process. These requirements led to the step-dither procedure described above: Images of fields belonging to a group are median-combined to produce a "scattered light frame," which is then rescaled and subtracted from each individual exposure before further processing and stacking (Figure 10). The associated processing pipeline, Elixir$L S B$, is significantly more complicated than described here, and is discussed in detail in Paper II of this series (C. Cuillandre et al. 2012, in preparation).

The step-dither procedure is not effective for the short exposures, for which the background $\mathrm{S} / \mathrm{N}$ is insufficient to 
characterize scatter light. Furthermore, the short exposures are used exclusively to recover the centers of galaxies that saturate in the long exposures. The Megapipe Global Background method, described in Section 4.2, reduces the scattered light bias to a level (a few percent of the sky value) that is entirely insignificant when studying high surface brightness objects, and is therefore adequate for processing the short exposures. All short exposures are therefore obtained according to a standard five-point dither patter, while the step-dither acquisition procedure described above is only used for the long exposures.

\section{DATA PROCESSING}

\subsection{Preprocessing}

All preprocessing of NGVS data is performed at CFHT. Both long and short exposures are processed using the Elixir reduction pipeline, which includes bad pixel masking, overscan and bias subtraction, flat fielding, and, in $i$ and $z$, an extra additive correction for fringing. A master flat field is applied to all MegaCam data obtain in a given run (typical MegaCam runs last for approximately two weeks each month, centered on the new moon) and is built by optimally combining twilight flats obtained, weather permitting, at the beginning and end of each night. The master flat field includes a correction for sensitivity variations due to non-uniform transmissions in the optics, different electronic gains, and quantum efficiency variations. In addition, the master twilight flat field is convolved with a photometric response map that corrects for the photometric bias introduced by the geometric distortion (which causes the pixel scale, and therefore the flux of astronomical objects, to vary across the mosaic) and by variations in the filter bandpass across the mosaic. The photometric response map renders the photometry of all the stars uniform regardless of their position on the image, and is built by measuring the flux of thousands of stars on a frame processed with a plain twilight flat, as they are moved across the mosaic. The resulting master twilight flat fields are such that Elixir delivers images that are photometrically flat across the entire mosaic. The correction for fringing is based on a linear scaling and subtraction of a master fringe pattern build from a large number of science frames taken at as many different sky locations as possible. Elixir also provides a preliminary astrometric calibration for each MegaCam CCD using the standard USNO astrometric catalog, and (for data taken in photometric conditions) an instrumental $\mathrm{AB}$ photometric calibration based on all standard star fields taken throughout a single MegaCam run. More accurate astrometric and photometric calibrations are derived during the stacking procedure described in Section 4.2.

Following this first traditional step through Elixir, which delivers a photometrically flat and calibrated image, all long exposures, in every filter, are then run through a second reduction pipeline, Elixir- $L S B$, designed specifically to correct for contamination by scattered light, as discussed in Section 3.5, and restore the true uniform sky background through a pure subtraction process that leaves the uniform photometry produced by Elixir untouched. The Elixir- $L S B$ pipeline, which is described in detail in C. Cuillandre et al. (2012, in preparation; Paper II of this series), was designed specifically for the NGVS, although it can be applied to any MegaCam data that adopt the NGVS step-dither procedure. Directly inspired by standard infrared observing techniques, though for slightly different reasons, Elixir$L S B$ produces a "scattered light" image by combining, after applying optimal filtering and scaling based on individual sky levels, frames of different fields taken as part of an uninterrupted observing sequence. An optimized sigma clipping algorithm is applied when combining frames, and the resulting image is then median-filtered and Gaussian-smoothed before being rescaled and subtracted from each of the individual frames. Typical peakto-peak residuals in the final scattered-light-subtracted images are $0.2 \%$ of the sky background in all filters (with peak-to-peak excursions of up to $0.5 \%$, but affecting only a small fraction of the mosaic as a result of too many extended objects falling on the same location of the mosaic in each input image), allowing the recovery of surface brightness features almost 7 mag fainter that the sky. The fundamental limitations to reaching even fainter limits stem from crowding in individual exposures and natural variations in the sky background during the sequence. In the $z$ band, the correction for fringing is intentionally not applied during the Elixir processing; instead, an optimized smoothing scale applied to the combined image allows an improved fringing correction over what is achieved by the Elixir pipeline. A comparison of the raw, Elixir and Elixir-LSB frames for the NGVS-1-4 field that includes M49 is shown in Figure 11. The radial scattered light component is clearly seen in the Elixir processed frames, but is entirely removed by the Elixir- $L S B$ processing. One last, but important, feature of the Elixir-LSB pipeline is that it performs a strict quality control on the images, to ensure that they satisfy the observing constraints discussed in Section 3.5. Any frame found to be out of specifications (a rare occurrence) is then re-observed.

\subsection{Image Stacking, Astrometric, and Photometric Calibration}

For most scientific applications, dithered images of each field must be combined or "stacked." The final data products then consist of five long and five short science images for each field (one per filter), plus associated weight maps. The primary stacking for the NGVS is performed at NRC-HIA using a variant of the MegaPipe pipeline (Gwyn 2008). As this will be described in detail in J. Gwyn et al. (2012, in preparation; Paper III of this series), only a few essential details are presented here. Additional stacks, designed to optimize $\mathrm{S} / \mathrm{N}$ for color and shape measurements of small and faint background galaxies for lensing and photometric redshift studies, are produced at the University of British Columbia using the THELI ${ }^{40}$ pipeline (Erben et al. 2005, 2009), and will be described in a separate paper of this series. Finally, independent stacking efforts are underway at TERAPIX (Bertin et al. 2002); these will also be described in a future contribution.

Starting from Elixir or Elixir-LSB preprocessed frames, MegaPipe cross correlates each frame against the SDSS DR7 astrometric and photometric catalogs to produce astrometric and photometric solutions independently for each CCD (with one exception, mentioned below). The astrometric solution has an internal accuracy of $0{ }^{\prime} 04$, measured by comparing positions of stars in the overlap region between adjacent fields, while the residuals with respect to the SDSS are 0 ' 15 . The photometric zero points are accurate to $1 \%$ both internally (as measured between overlapping pointings) and externally (with respect to the SDSS).

Four separate stacks are then produced for each field/filter combination, according to the methodology adopted to subtract the background and combine the frames (see the bottom row of Figure 11).

\footnotetext{
40 See http://www.astro.uni-bonn.de/theli/.
} 
(a) Raw Frame

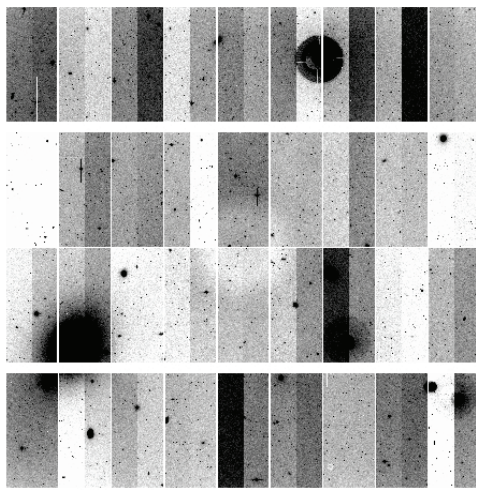

(d) MegaPipe Local Background Stack

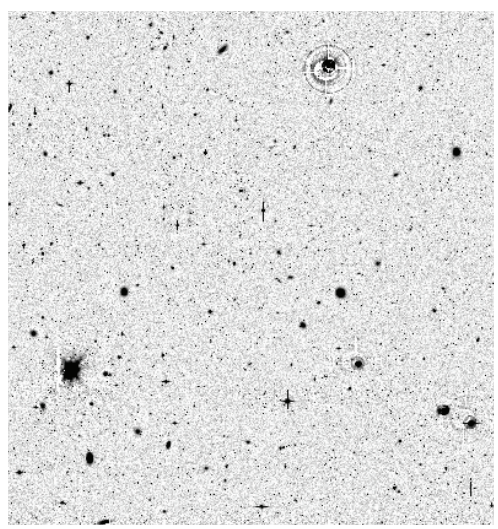

(b) Elixir Processed Frame

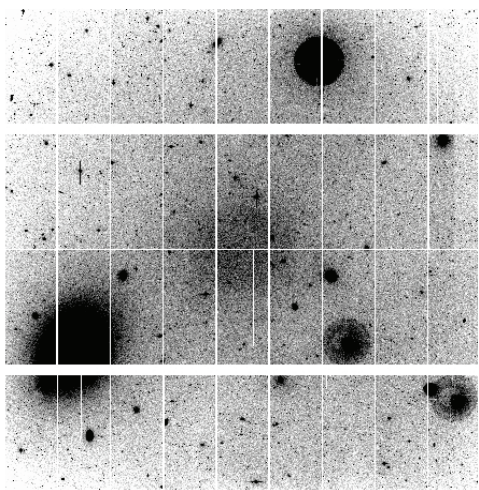

(e) MegaPipe Global Background Stack

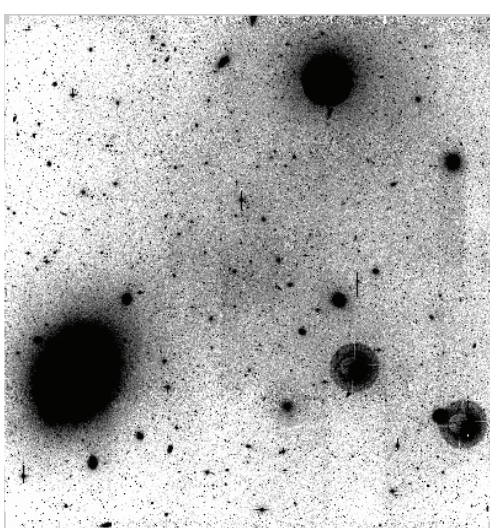

(c) Elixir-LSB Processed Frame

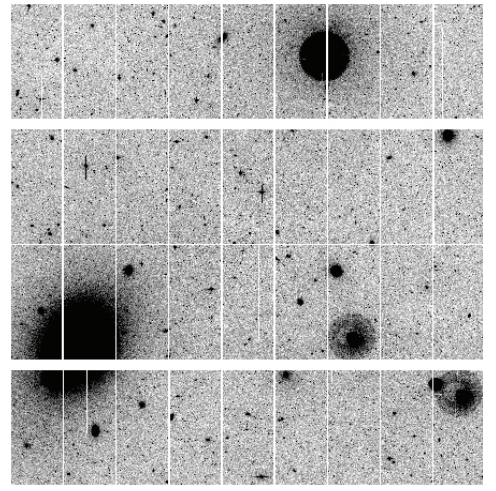

(f) MegaPipe Elixir-LSB Stack

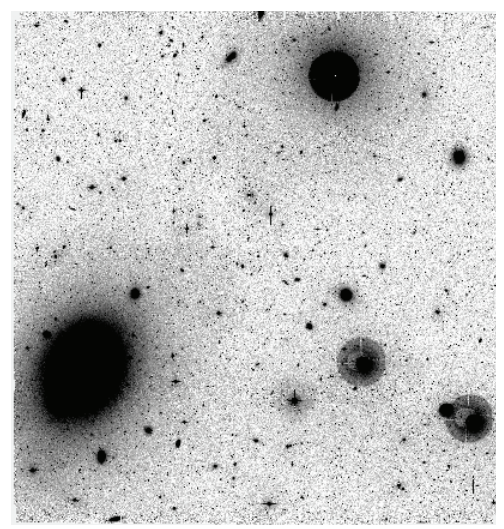

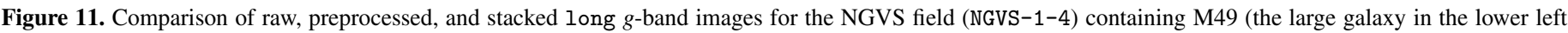

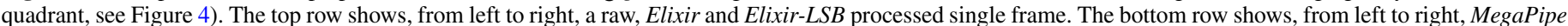

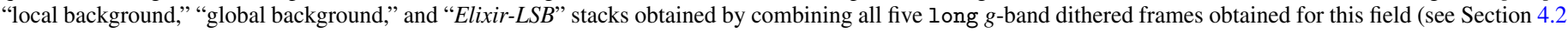
for further details). All panels use a similar gray scale.

1. "Local background stacks." A local estimate of the background for each Elixir preprocessed frame is produced using the SWarp software (Bertin et al. 2002). To properly model the scattered light pattern, the background is estimated in a $128 \times 128$ pixel grid. As a result, any objects extending on scales larger than several arcseconds (including all but the faintest and most compact of Virgo galaxies) are incorporated in the background and partially subtracted. While this is obviously undesirable, this is the standard procedure that was applied to all MegaPrime images prior to the introduction of the NGVS step-dither pattern and the development of Elixir-LSB. The same pipeline is applied to the NGVS images, not only to provide consistency with existing data sets, but also because it produces superior photometric accuracy for compact and point sources compared to the "global background" and "Elixir-LSB" stacks described below. Background-subtracted images are then resampled to a uniform pixel scale using SWarp, and then median-combined to produce a final stack and associated weight map.

2. "Global background stacks.” A global estimate of the background, produced by median-combining archival Elixirprocessed MegaPrime images, is scaled to the appropriate background level and subtracted from each NGVS Elixirprocessed frame. The resulting frames are then rescaled and median-combined. Because of the temporal variation in scattered light, residuals in the resulting stacks can be several percent of the sky background. Although these stacks are largely superseded by the Elixir- $L S B$ stacks described below, they have higher photometric accuracy than the Elixir-LSB stacks, and are therefore better suited for some applications (e.g., GCs photometry). Furthermore, the methodology continues to be supported since it represents a significant improvement over the "local background stacks" for observations of extended objects, when the data are taken in standard dither mode, or in conditions that do not allow the application of Elixir-LSB.

3. "Elixir-LSB stacks." The spatially varying component of the background has already been removed in frames processed with Elixir-LSB (which is precisely the point of the Elixir-LSB processing). A constant pedestal representative of the sky brightness is preserved in the data since it is instrumental to understand and check the $\mathrm{S} / \mathrm{N}$ properties and derive accurate quality control metrics for the flattening of the data. A constant sky background is therefore computed for, and subtracted from, each Elixir-LSB processed frame before the frames are rescaled and combined. Two separate methods are used to combine the frames: a standard median (implemented within SWarp) and a stand-alone implementation of the artificial skepticism method (Stetson 1989). The latter computes a robust average image using a continuous weighting scheme that is derived from the data themselves through an iterative process. Artificial skepticism produces stacks with superior $\mathrm{S} / \mathrm{N}$ properties (an improvement in depth of $\sim 0.15 \mathrm{mag}$ ) compared with a median. At the time of writing, the photometry of Elixir- $L S B$ 
stacks is not as tight as in the "local background" stacks. This is due to the fact that when using frames processed through Elixir- $L S B$, the photometric calibration cannot be performed independently for each CCD (as is done in the case of the "local background" stacks), since a different scaling factor applied to different CCDs would translate in discontinuities in the sky level across CCD boundaries. A single photometric correction is therefore calculated and applied across the entire mosaic, with the consequence that small photometric zero-point variations from CCD to CCD are not corrected. The photometry in Elixir-LSB stacks therefore displays variations of up to $0.03 \mathrm{mag}$, compared with the 0.01 mag dispersion for Elixir "local" and "global" background stacks. However, this situation will soon be corrected. In the context of the CFHT Legacy Survey photometric accuracy investigations (Regnault et al. 2009), Elixir products were scrutinized and improvements to the photometric correction map were adopted by CFHT, bringing the overall non-uniformity of the zero points between CCDs down from $\sim 3 \%$ to $<1 \%$. In the public release of ElixirLSB NGVS stacks (expected in 2014) the photometry will be comparable to the "local background" stacks which rely on individual rescaling of each CCD zero point based on the SDSS.

A comparison of "local background," "global background," and Elixir-LSB MegaPipe stacks for the NGVS-1-4 field is shown in the bottom row of Figure 11. While the "local background" stacks appear to provide a very uniform background, any extended structure is incorporated in the background maps and subtracted, with the consequence that large-scale structures (whether they are galaxies, tidal features, or scattered light) are erased (including, in the case shown in the figure, M49 itself). The "global background" stacks preserve some of these features, but it is only the Elixir-LSB stacks that allow a full exploitation of the surface brightness levels achievable by the data. Note that for the short exposures, which are not observed in the proper sequence for Elixir- $L S B$ processing, only "local" and "global" background stacks are available.

All NGVS images are scaled to a photometric zero point of 30.0 , such that $\mathrm{AB}$ magnitudes $m(\mathrm{AB})$ are given by

$$
m(\mathrm{AB})=-2.5 \times \log (\mathrm{DN})+30.0,
$$

where DN is the number of counts measured in the frame. The magnitudes thus obtained are on the MegaPrime photometric system, which is related to the SDSS system by the following relations ${ }^{41}$ :

$$
\begin{gathered}
u_{\mathrm{MegaCam}}=u_{\mathrm{SDSS}}-0.241\left(u_{\mathrm{SDSS}}-g_{\mathrm{SDSS}}\right) \\
g_{\mathrm{MegaCam}}=g_{\mathrm{SDSS}}-0.153\left(g_{\mathrm{SDSS}}-r_{\mathrm{SDSS}}\right) \\
r_{\mathrm{MegaCam}}=r_{\mathrm{SDSS}}-0.024\left(g_{\mathrm{SDSS}}-r_{\mathrm{SDSS}}\right) \\
i_{\mathrm{MegaCam}}=i_{\mathrm{SDSS}}-0.003\left(r_{\mathrm{SDSS}}-i_{\mathrm{SDSS}}\right) \\
z_{\mathrm{MegaCam}}=z_{\mathrm{SDSS}}+0.074\left(i_{\mathrm{SDSS}}-z_{\mathrm{SDSS}}\right) . \\
\text { 4.3. Additional Data Processing and Products }
\end{gathered}
$$

\subsection{Additional Data Processing and Products}

Each of the 117 NGVS fields produces 61 raw frames (25 of which are associated with short exposures), 61 Elixir processed frames, and 36 Elixir-LSB processed frames (for the long

\footnotetext{
41 These equations will change for the final public release of NGVS data, which will adopt the photometric calibration described in Regnault et al. (2009).
}

exposures only). These are combined to give 55 stacked images per field: 10 for the short exposures (five each from MegaPipe and TERAPIX), and 45 for the long exposures (nine for each filter, see also Figure 12). Two additional sets of stacks per filter, binned by $3 \times 3$ and $24 \times 24$ pixels, are generated for quality control during the Elixir-LSB processing. Each stack has associated weight maps. To date, the NGVS has generated 10 Terabytes of data, a volume similar to that produced by the imaging component of the SDSS. All data are stored at the Canadian Astronomy Data Centre (CADC) and available to the collaboration through a dedicated web interface.

Several NGVS science goals require additional data products. In particular:

1. Many applications (for instance, fitting structural parameters to Virgo galaxies) require masking contaminant objects, in particular bright stars and their associated diffraction spikes. Masks identifying stars brighter than a given magnitude limit in the Guide Star Catalog (GSC) are created using the THELI "automask" software, modified to identify, trace and mask spikes and bleed regions around the stars.

2. Point-spread functions (PSFs) are needed to recover the intrinsic structure of galaxy cores, stellar nuclei, and compact objects, and are a critical component in the weak-lensing analysis. PSFs are generated using two independent procedures. The first, which will be described in detail in J. Gwyn et al. (2012, in preparation; Paper III) uses an iterative, automated version of DAOPhot (Stetson 1987) which relies on SExtractor parameters (Bertin \& Arnouts 1996) to identify a list of uncrowded stars across the field. The second is based on Shapelet (Refregier 2003) decomposition of stars, again selected based on SExtractor parameters, followed by a fit of the Shapelet coefficients over the entire mosaic to enable the reconstruction of model PSFs at any given position.

3. Although Virgo is at relatively high Galactic latitude, halos around bright stars - due to both internal reflections within the instrument, and the extended stellar PSF-ultimately limit the ability to trace the diffuse ICL within the cluster and, in some cases, derive accurate structural parameters for galaxies. Efforts are underway to subtract stellar halos by modeling each reflection as a constant surface brightness ring, whose spatial offset from its parent star is parameterized as a function of the star's position on the chip. After modeling and subtracting each reflection, the star's extended PSF is modeled and subtracted as a higher-order radial polynomial. A dedicated imaging program is underway at CFHT in which a bright star is imaged multiple times across the mosaic, making it possible to model the larger, fainter reflections.

\subsection{Survey Timeline}

The NGVS was designed as a four-year survey; data collection started in the spring of 2009 and will conclude in 2013 January. Throughout the observations, priority was given to the $u^{*}-, g-$, and $i$-band data, with the goal of achieving complete spatial coverage in $g$ and $i$ by the end of the second year. The coverage as of 2011 September is shown in Figure 13; although the entire $104 \mathrm{deg}^{2}$ survey area has been imaged in $g$, due to exceptionally bad weather on Mauna Kea in the 2009 and 2011 spring semesters, spatial coverage in the other filters (and in the short exposures) is not yet complete. To compensate for time 


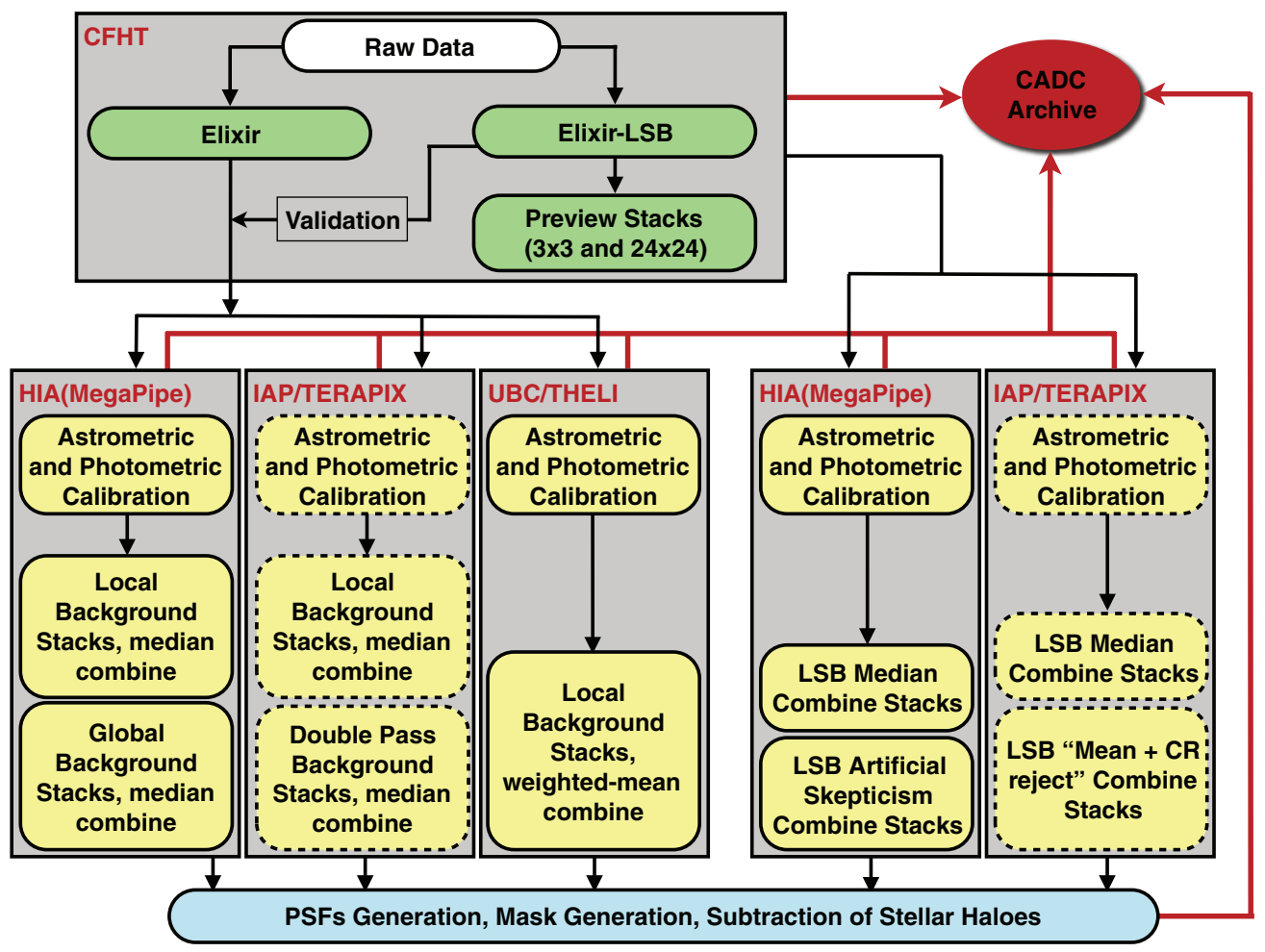

Figure 12. Schematic representation of the NGVS data flow. Quality control and preprocessing of raw data is performed at CFHT using two separate pipelines: the standard MegaPrime pipeline Elixir and Elixir-LSB, designed to take advantage of the NGVS step-dither observing strategy (see Section 3.5). Astrometric, photometric calibration, and stacking of Elixir frames are performed using three independent pipelines, MegaPipe, TERAPIX (in progress, and shown by the dashed lines), and THELI. Astrometric, photometric calibration, and stacking of Elixir-LSB frames are performed using MegaPipe and TERAPIX (see Section 4.2 for details). All data, from raw frames to final calibrations and data products, are stored at the CADC.

(A color version of this figure is available in the online journal.)

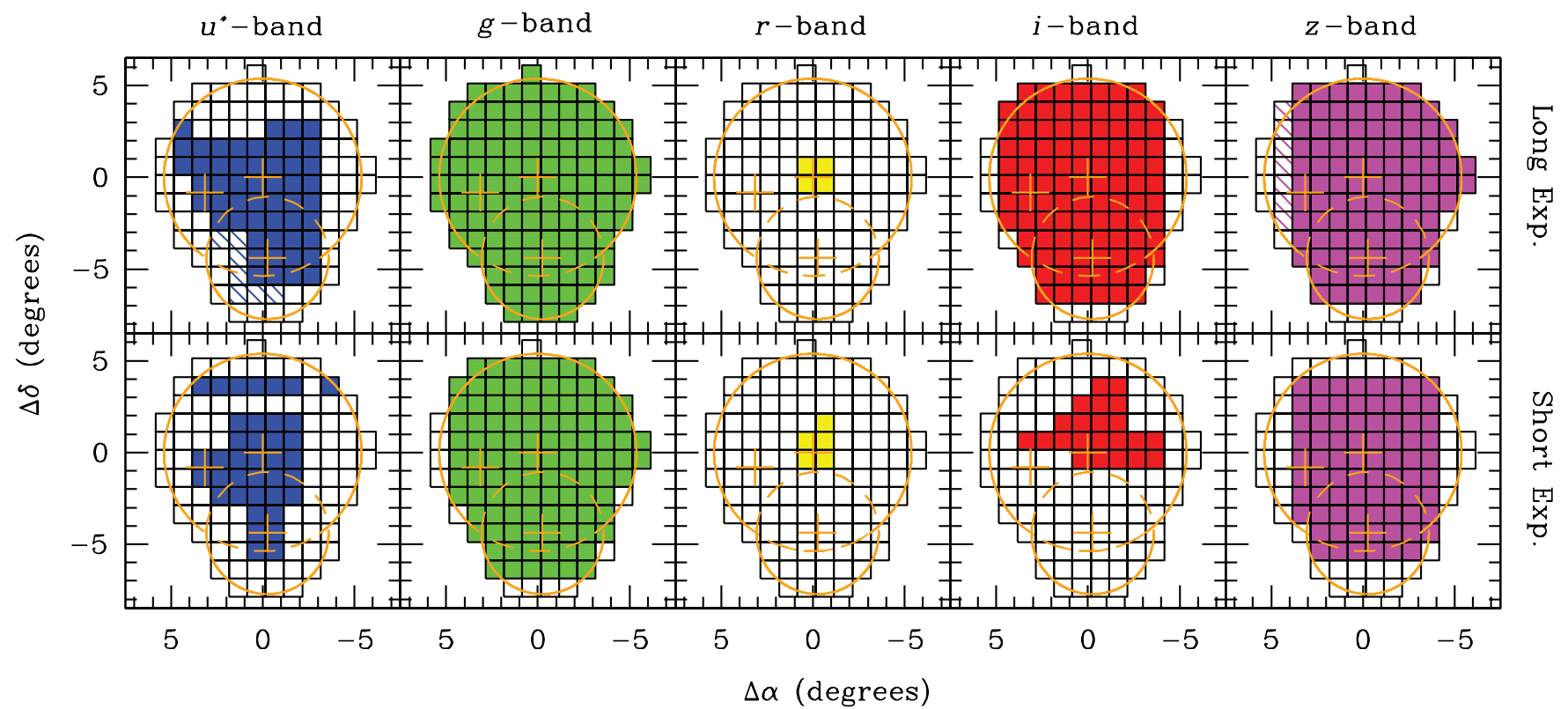

Figure 13. NGVS observing status as of 2011 September, i.e., at the end of year three. Long and short exposures are shown in the top and bottom rows, respectively, while the filters are ordered from $u^{*}$ to $z$ from left to right. Fields for which observations have been completed are indicated by colored filled squares, while only partial data have been obtained for dashed fields. Fields shown as empty squares will be observed in 2012 or beyond. The orange outline indicates the virial radii of Virgo's A and B subclusters, while the crosses mark the location of M87 to the north, M49 to the south, and M60 to the east.

(A color version of this figure is available in the online journal.)

lost to weather, in $r$ only shallow exposures (1374 s instead of the original 4908 s) will be obtained in 2012/2013. Although these exposures are too shallow for the study of extended, low surface brightness features in Virgo (a task that can, however, be performed using the $g$-band images), they are of sufficient depth to allow us to measure photometric redshifts with a precision 
adequate for weak-lensing studies (Section 5.3.1), and to apply the red-sequence technique for the detection of galaxy clusters in the $0.4 \lesssim z \lesssim 0.8$ redshift range (Section 5.3.2).

\section{SCIENTIFIC MOTIVATION}

Having reviewed the technical and programmatic elements of the NGVS, we now turn to a description of the survey's science drivers, beginning with those involving the Virgo cluster itself (Section 5.1) and continuing on to a number of "foreground" and "background" science topics (Sections 5.2 and 5.3).

\subsection{Virgo Cluster Science}

\subsubsection{The Galaxy Luminosity Function}

The galaxy luminosity function, $\phi(L)$, is one the most fundamental observational constraints on cosmological models of galaxy formation. Characterizations of the luminosity function date back many decades, with early "bell-shaped" distributions (Hubble \& Humason 1931) eventually giving way to forms with a sharp cutoff at high luminosity and a power-law behavior at the faint end (e.g., Zwicky 1942, 1957; Holmberg 1969; Schechter 1976). Determinations of the luminosity function have improved dramatically in accuracy as redshift surveys have grown in size (e.g., Efstathiou et al. 1988; Loveday et al. 1992; Marzke et al. 1994; Lin et al. 1996; Cole et al. 2001; Norberg et al. 2002; Blanton et al. 2003). In conjunction, there has been steady progress in our theoretical understanding of the physical processes that shape the luminosity function. For instance, the sharp downturn at high luminosities is currently thought to be the result of suppressed cooling in massive halos, due, for example, to gas expulsion or thermal conduction. The relatively flat faintend slopes (compared with the $\Lambda$ CDM halo mass function) are usually attributed to photoionization or suppressed cooling in this regime (see Benson et al. 2003, and references therein), although the disagreement between observations and predictions at the low-mass end is so extreme (e.g., Klypin et al. 1999; Moore et al. 1999; but see also Tollerud et al. 2008) that alternative scenarios-including modifying the power spectrum on small scales or changing the properties of DM particles themselves-have also been explored in considerable detail.

A dramatic leap forward in our understanding of the luminosity function in the local universe came in the 1980s, with the Virgo cluster survey of Binggeli and co-workers. Indeed, this survey was largely motivated by the desire to exploit the richness and proximity of Virgo in an accurate measurement of $\phi(L)$, both for the ensemble of galaxies, as well as a function of Hubble type (Sandage et al. 1985). These authors found a Schechter function form,

$$
\phi\left(M_{B}\right) d M \propto \phi(L) d L / L \propto 10^{0.4\left(M_{B}^{*}-M_{B}\right)(\alpha+1)} d M,
$$

with $M_{B}^{*}=-20.87 \mathrm{mag}$ and $\alpha=-1.30$ when including galaxies of all Hubble types. ${ }^{42}$ Somewhat steeper faint-end slopes were obtained when the analysis was restricted to early-type galaxies, reaching a maximum of $\alpha=-1.45$ for a subsample consisting of $\mathrm{E}+\mathrm{dE}$ galaxies only. These luminosity functions, although significantly steeper than those found in the Local Group, remain shallower than those predicted for $\mathrm{DM}$ halos in $\Lambda \mathrm{CDM}$ cosmologies.

\footnotetext{
42 Here, $M_{B}^{*}$ has been calculated using the value of $B^{*}=10.3 \mathrm{mag}$ from Sandage et al. (1985), a mean $B$-band absorption of $A_{B}=0.08 \mathrm{mag}$, and the modern SBF distance to Virgo of 16.5 Mpc from Mei et al. (2007).
}

Perhaps surprisingly, the study of Sandage et al. (1985) probably remains the most reliable measurement currently available for the global luminosity function in a low-redshift cluster, reaching simultaneously to $M_{B} \approx-13$ and $\left\langle\mu_{B}\right\rangle_{e} \approx$ $25.3 \mathrm{mag} \mathrm{arcsec}^{-2}$ with a high level of completeness. There have been many subsequent investigations of the faint end of the luminosity function in Virgo, including those of Impey et al. (1988), Phillipps et al. (1998), Trentham \& Hodgkin (2002), Sabatini et al. (2003), and Rines \& Geller (2008). A comparison of these luminosity functions is presented in Figure 14. Note that the areal coverage in these studies is typically much smaller than that of Sandage et al. (1985). To roughly account for this difference, we have scaled the counts from the later studies by the ratio of survey area to that of the VCC. Note that this procedure can overpredict, or underpredict, the true normalization depending on the average density of the region explored in any particular survey.

Such details aside, this comparison shows that there are significant discrepancies between the different studies below $B \sim 17$, with faint-end slopes ranging from $\alpha \simeq-1.3$ to -2.2 due, at least in part, to differences in the statistical treatment of background contamination adopted in the various studies. The NGVS-which has a $5 \sigma$ limiting magnitude for point sources of $g \approx 25.9$ and a $2 \sigma$ surface brightness limit of $\mu_{g} \sim$ 29.0 mag arcsec ${ }^{-2}$ — will supersede all previous measurements of $\phi(L)$ in the Virgo cluster, and indeed, in any low-redshift cluster. Figure 15 illustrates the power of the NGVS in this regard, showing a $10^{\prime} \times 10^{\prime}$ region in the cluster core. The two prominent galaxies indicated by cyan ellipses are both identified in the catalog of Binggeli et al. (1985); in particular, VCC1129, with $B=17.69 \mathrm{mag}$, is close to the completeness limit of the VCC. Seven low-mass galaxies not cataloged in the VCC but readily apparent in the NGVS images are shown in yellow. Of these, only one (the brighter galaxy to the southeast of VCC1129) was detected based on the deep optical data presented in Lieder et al. (2012). The newly discovered NGVS galaxies have magnitudes as faint as $g=24.9\left(M_{g} \simeq-6.2\right.$ if in Virgo) and average surface brightnesses within one effective radius as low as $\left\langle\mu_{g}\right\rangle_{e} \approx 28.8 \mathrm{mag} \mathrm{arcsec}^{-2}$. Although, in the absence of spectroscopic information, the cluster membership of these galaxies is not unambiguous, their sizes, surface brightness, and colors all indicate that they very likely belong to the cluster. Because multi-band photometry will be available for all galaxies in the NGVS, it will also be possible to measure rough stellar mass-to-light ratios on a galaxy-by-galaxy basis using standard procedures (e.g., Bell et al. 2003). This will allow a direct estimation of the galaxy mass function, $\phi\left(\mathcal{M}_{*}\right)$, down to $\mathcal{M}_{*} \approx 10^{5}-10^{6} \mathcal{M}_{\odot}$ for dwarf galaxies (and star clusters; see Section 5.1.6).

\subsubsection{The Color-Magnitude Diagram of Galaxies at $z \approx 0$}

Pioneering photometric studies established the existence of a relationship between luminosity and color for early-type galaxies, in the sense that high-luminosity objects tend to be redder than their low-luminosity counterparts (e.g., Baum 1959; de Vaucouleurs 1961b). Intermediate-band photometry for $\approx 30$ ellipticals (Faber 1973b) later revealed this CMR to be extremely tight at the high-luminosity end (with little or no dependence on environment), demonstrating the importance of luminosity (or, alternatively, mass) in dictating the history of star formation and chemical enrichment within individual galaxies (e.g., Gavazzi et al. 1996). Subsequent photometry of earlytype galaxies in the Virgo cluster showed that E and SO galaxies 


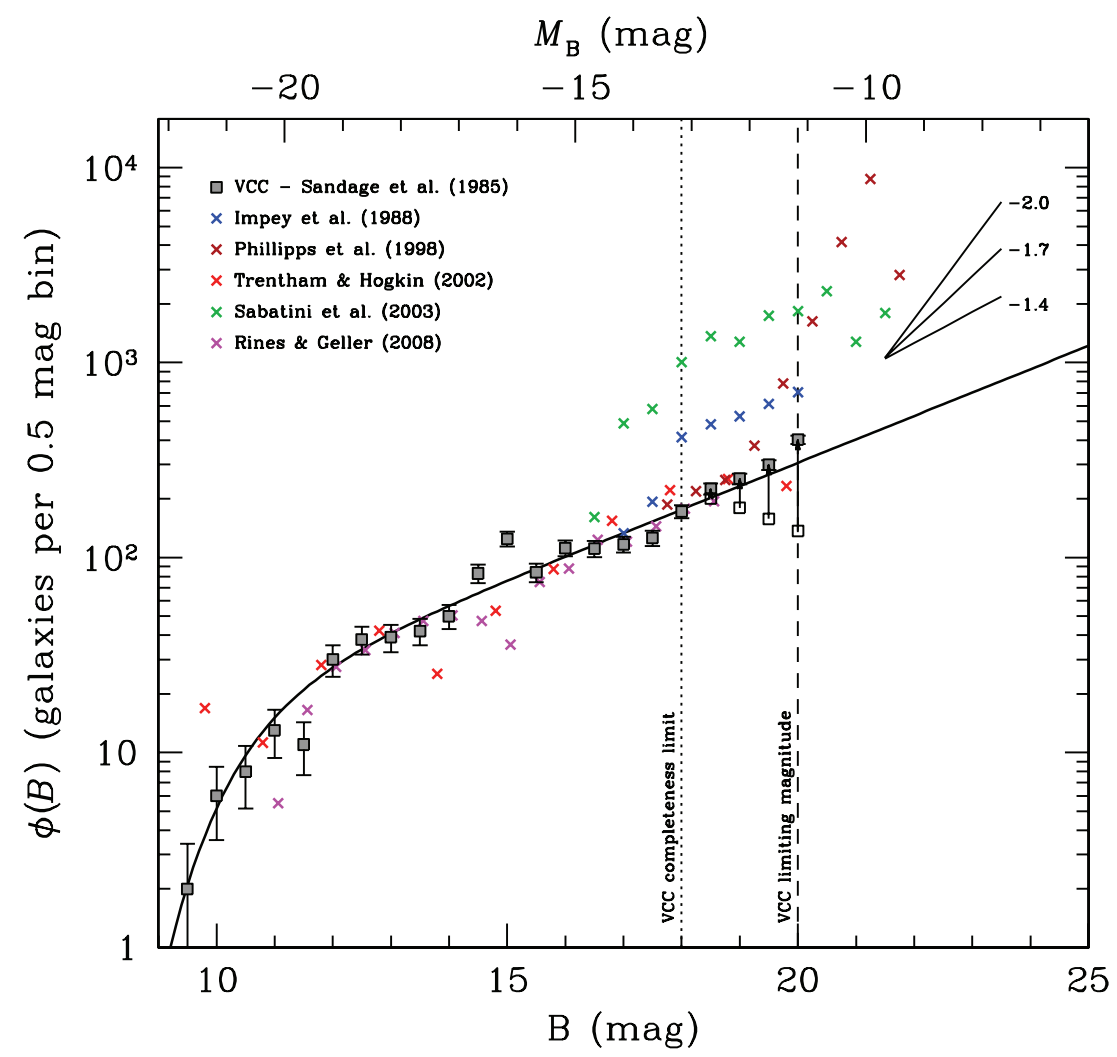

Figure 14. $B$-band luminosity function of the Virgo cluster from Sandage et al. (1985) compared to more recent measurements. The solid curve is the best-fit Schechter function $(\alpha=-1.3)$ obtained for the entire cluster and all galaxy types (Sandage et al. 1985). As subsequent studies have typically surveyed only a fraction of the cluster, these measurements have been normalized by area to compare with those of Sandage et al. (1985). The exception is the study of Rines \& Geller (2008) which relied on SDSS spectroscopy of galaxies within $1 \mathrm{Mpc}$ of M87. There is significant scatter in the luminosity functions fainter than $B \sim 17$ mag, with published faint-end slopes ranging from $\alpha \approx-1.3$ to -2.2 . As it is based entirely on SDSS radial velocities, the value of $\alpha=-1.3$ from Rines \& Geller (2008) is a firm lower limit on the faint-end slope down to $B \sim 18 \mathrm{mag}\left(M_{\mathrm{B}} \sim-13 \mathrm{mag}\right)$, although brighter, but low surface brightness members could also have been excluded in the SDSS spectroscopic survey.

traced out a narrow sequence that was offset from the broader (and bluer) distribution of late-type systems within the CMD (i.e., Visvanathan \& Sandage 1977; Sandage \& Visvanathan 1978).

In recent years, the distribution of galaxies within the CMD has become a powerful tool for understanding the formation and evolution of galaxies. With the availability of precise-and homogeneous - photometry for very large samples of galaxies from the SDSS (e.g., Strateva et al. 2001; Bell et al. 2004; Baldry et al. 2004, 2006), the CMD has been used to identify distinct subpopulations of galaxies, independent of classical Hubble types (i.e., the so-called red sequence, blue cloud, and green valley components). Such classification schemes are now used routinely to subdivide samples in the measurement of key observables, including galaxy luminosity functions, correlation functions, and scaling relations. The general picture to emerge from the many studies of the CMR over a range of redshifts and in different environments is that the red-sequence population is built up through a combination of merging and gas depletion (i.e., stellar and AGN feedback, inefficient gas cooling, etc.), with the relative importance of these process likely varying as a function of mass (see, e.g., Faber et al. 2007, and references therein), and environment (e.g., Hughes \& Cortese 2009; Gavazzi et al. 2010).

To date, even the most ambitious studies of the CMD have tended to focus on high- and intermediate-luminosity galaxies (e.g., Faber et al. 2007; Brown et al. 2007; Cassata et al. 2007; Mei et al. 2009). The NGVS presents a unique opportunity to extend these studies in two important respects. First, because of Virgo's proximity, it is possible to characterize the CMD down to a luminosity regime that is completely inaccessible in more distant systems; i.e., depending on the surface brightness selection criteria, the NGVS will give complete coverage of the CMD down to $-8 \lesssim M_{g} \lesssim-6$ (assuming the trend shown by the red line in Figure 16). Second, with a physical resolution of $\approx 50 \mathrm{pc}$, it will be possible to derive morphological parameters for individual objects-and accurately quantify the likelihood of past mergers, interactions, central starbursts, or AGN activity - at a level of detail that will never be possible in more distant systems. One issue of particular interest is the precise form of the CMR at the low-mass end. Early studies suggested that these low-luminosity dwarfs follow a roughly linear CMR (Caldwell 1983; Prugniel et al. 1993) that merged smoothly with that of the high-mass galaxies (e.g., Secker et al. 1997; Conselice et al. 2002; Karick et al. 2003). SDSS photometry for early-type galaxies in Virgo suggests that the CMR is indeed continuous (Janz \& Lisker 2009; see also Ferrarese et al. 2006a) to $M_{g} \approx-13.5$ (equivalent to a stellar mass of $\sim 10^{8} \mathcal{M}_{\odot}$ ), but with a level of nonlinearity that depends on the color indices used to characterize the CMR (see Figure 17 of Chen et al. 2010). Given the wide range in local density probed by the NGVS fields, it will also be possible to provide a first characterization of the faint-end behavior of the CMR as a function of environment.

Moving beyond integrated colors, there is much to be learned about stellar populations within individual galaxies from the 


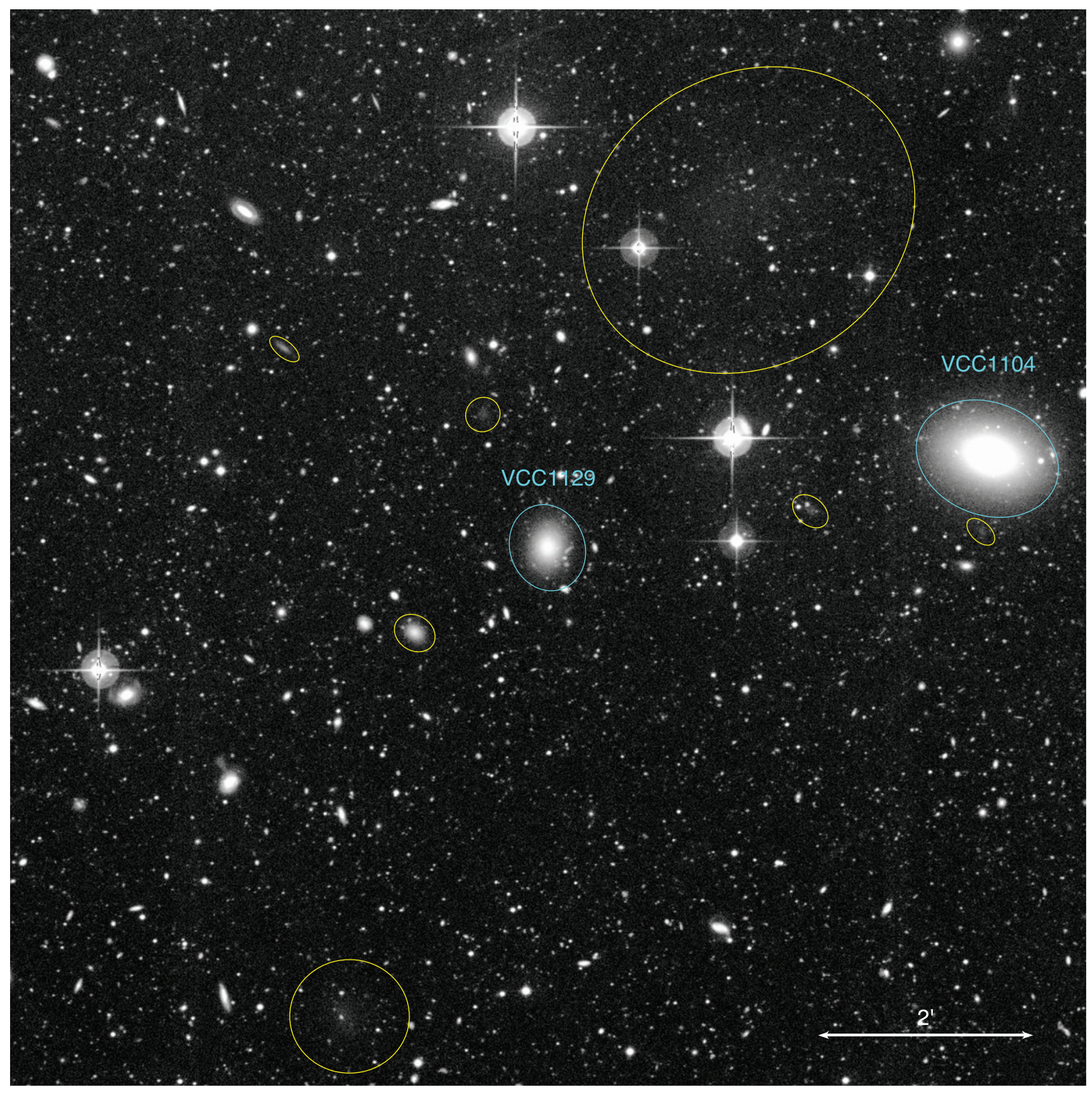

Figure 15. Magnified view of a stacked $g$-band NGVS image showing a $10^{\prime} \times 10^{\prime}$ region located in the Virgo cluster core. This field is located approximately $40^{\prime}$ $(\sim 200 \mathrm{kpc})$ to the northwest of M87. Two early-type dwarf galaxies from the VCC of Binggeli et al. (1985) are shown by the cyan ellipses: VCC1129 and VCC1104 ( $g \approx 17.7 \mathrm{mag}$ and $15.4 \mathrm{mag}$, respectively). The yellow ellipses show a number of faint, low surface brightness dwarfs that are probable cluster members based on sizes, surface brightness, and colors; the small galaxy to the southeast of VCC1129 (also detected by Lieder et al. 2012) has in fact recently been spectroscopically confirmed as a cluster member by our team. These newly discovered galaxies have magnitudes in the range $18.7 \mathrm{mag}<g<24.9$ mag and effective surface brightnesses of $24.3 \mathrm{mag} \operatorname{arcsec}^{-2}<\left\langle\mu_{g}\right\rangle_{e}<28.8 \mathrm{mag} \operatorname{arcsec}^{-2}$.

(A color version of this figure is available in the online journal.)

study of broadband colors on spatially resolved scales. Detailed studies of the resolved stellar populations in Virgo cluster galaxies have already uncovered several significant trends, such as that the chemical evolution of galaxies is driven by the interplay between stellar masses (nucleosynthesis sites) and surface densities (potential well depth), and that the stellar population gradients of most bright VCC galaxies exhibit little dependence on either their structure or environment (Roediger et al. 2011a, 2011b, and references therein). These and other modern stellar population studies have offered a new perspective on an array of problems related to galaxy formation and evolution, such as the formation of massive gas-poor galaxies (e.g., Kuntschner et al. 2010), the radial migrations of stars within gas-rich galaxies (e.g., Barker et al. 2011), and the multiple populations of dwarf galaxies within nearby galaxy clusters (e.g., Paudel et al. 2010), as well as the environmental mechanisms that quench star formation within gas-rich galaxies upon their infall into either galaxy groups or clusters (e.g., Crowl \& Kenney 2008).

Stellar population studies based on optical fluxes alone are hampered by the well-known degeneracy between stellar age and metallicity (Worthey 1994; but see also Tortora et al. 2010), and therefore the full potential of the NGVS in this respect will be exploited in combination with infrared imaging (see Section 2.2). However, thanks to its wide wavelength baseline ( $u^{*}$ to $z$ ), depth (spanning a factor $10^{7}$ in galaxy mass), and areal coverage (sampling galaxies within a wide range of local densities), the NGVS will provide insight into the stellar mass assembly history of galaxies, calibrate stellar population inferences based on optical data alone, and determine whether 


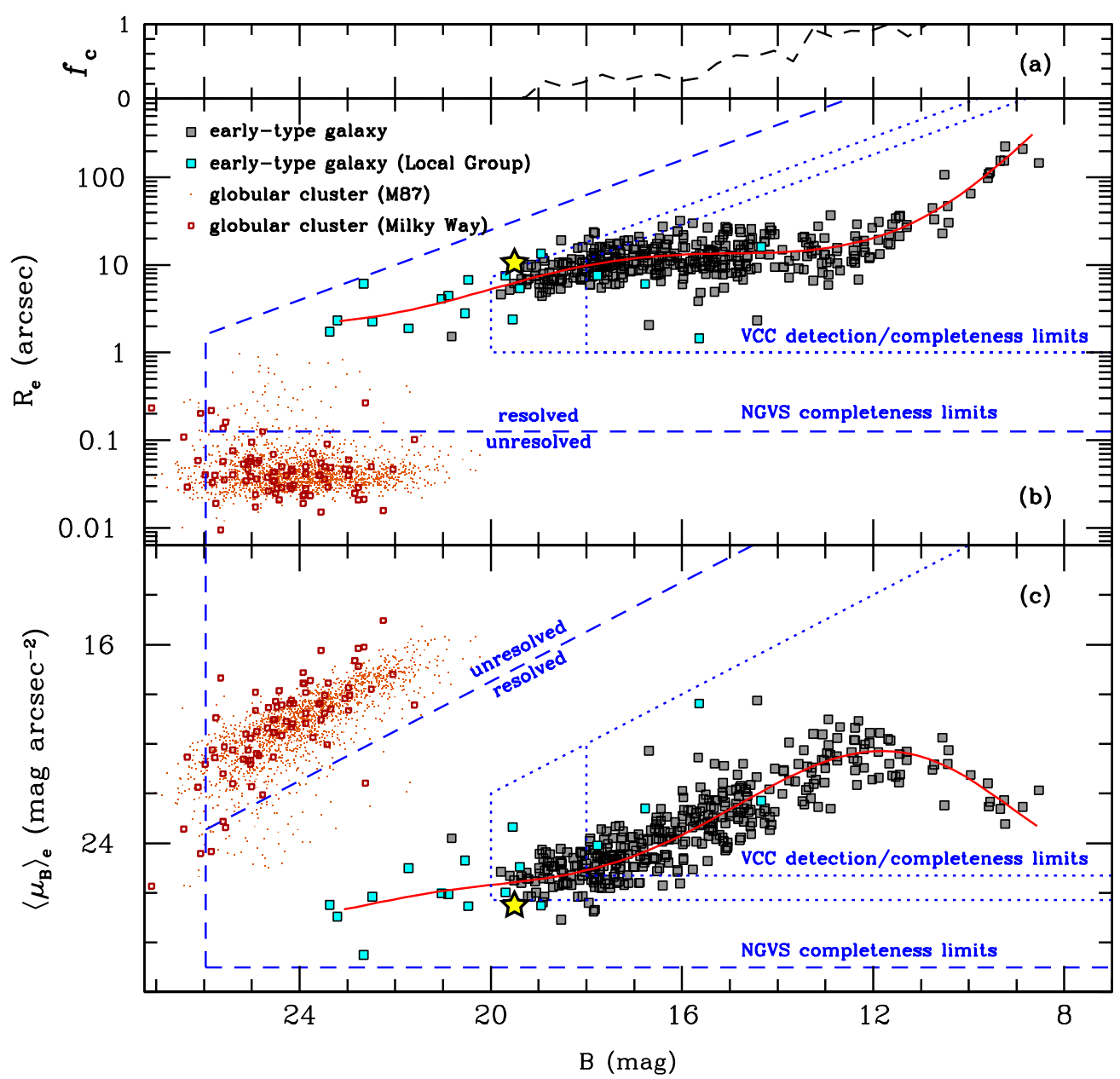

Figure 16. Middle and lower panels: scaling relations for early-type galaxies and globular clusters (GCs) in the Virgo cluster, Fornax cluster, and Local Group (see the text for details). For galaxies in Fornax and the Local Group, apparent $B$-band magnitudes and effective radii in arcseconds have been calculated as they would appear at the distance of the Virgo cluster. The dotted and dashed lines indicate the approximate selection limits for the Virgo survey of Binggeli et al. (1985) and the NGVS, respectively. The regions of parameter space where galaxies or star clusters in Virgo would be resolved are labeled, assuming an NGVS resolution limit of $R_{e}=10 \mathrm{pc}$ for compact sources (Section 5.1.4). The completeness function for the galaxy sample shown here is given in the top panel. The yellow star identifies the location of the galaxy shown in Figure 17.

stellar population diagnostics are consistent with other galaxy formation diagnostics, such as their structural and dynamical scaling relations (Section 5.1.3).

\subsubsection{Galaxy Scaling Relations: Structure, Photometry, and Dynamics}

While the galaxy luminosity function (Section 5.1.1) and CMD (Section 5.1.2) provide strong constraints on the global, macroscopic processes that governed the assembly of baryons within merging DM halos, it is the detailed structural, photometric, and dynamical scaling relations of galaxies that tell us about how these processes unfolded on galaxy scales. Indeed, the study of galaxy scaling relations has a rich history of yielding important, and often unexpected, insights into the formation and evolution of galaxies (e.g., Faber \& Jackson 1976; Tully \& Fisher 1977; Dressler et al. 1987; Djorgovski \& Davis 1987). Despite these successes, there are some important gaps in our present understanding of galaxy scaling relations, and what they can tell us about galaxy formation.

First, until recently most studies have tended to focus on high-mass galaxies, due to the technical difficulties of obtaining accurate photometric and kinematic data for low-mass, low surface brightness systems. Second, in constructing and inter- preting scaling relations for local galaxies, it is customary to restrict the sample to objects conforming to a pre-defined set of unique morphological properties: e.g., star-forming, rotationally supported spirals versus quiescent, rotationally flattened lenticulars versus quiescent, or dynamically hot ellipticals (a notable counterexample is the ATLAS3D survey, which analyzed earlytype galaxies selected largely independently of morphology; Cappellari et al. 2011a, 2011b; Emsellem et al. 2011; FalcónBarroso et al. 2011). The reasons for this reliance on morphological classification are both practical (i.e., the need to limit the sample size) and historical (i.e., galaxies belonging to different morphological classes are thought to have followed unrelated formation paths). However, the physical processes responsible for the observed relations include a number of mechanisms that can, in principle, operate on the progenitors of both early- and late-type galaxies (e.g., mergers, accretions, tidal torques, gas inflows/outflows, triggered star formation, cold gas accretion, stellar/AGN feedback, disk instabilities, tidal stripping, and harassment; see, e.g., Parry et al. 2009). Some of these processes can be highly effective in transforming galaxy morphology, hence the obvious need to move beyond the "classical" methodology in which galaxies are divided by morphology into a handful of distinct categories. 

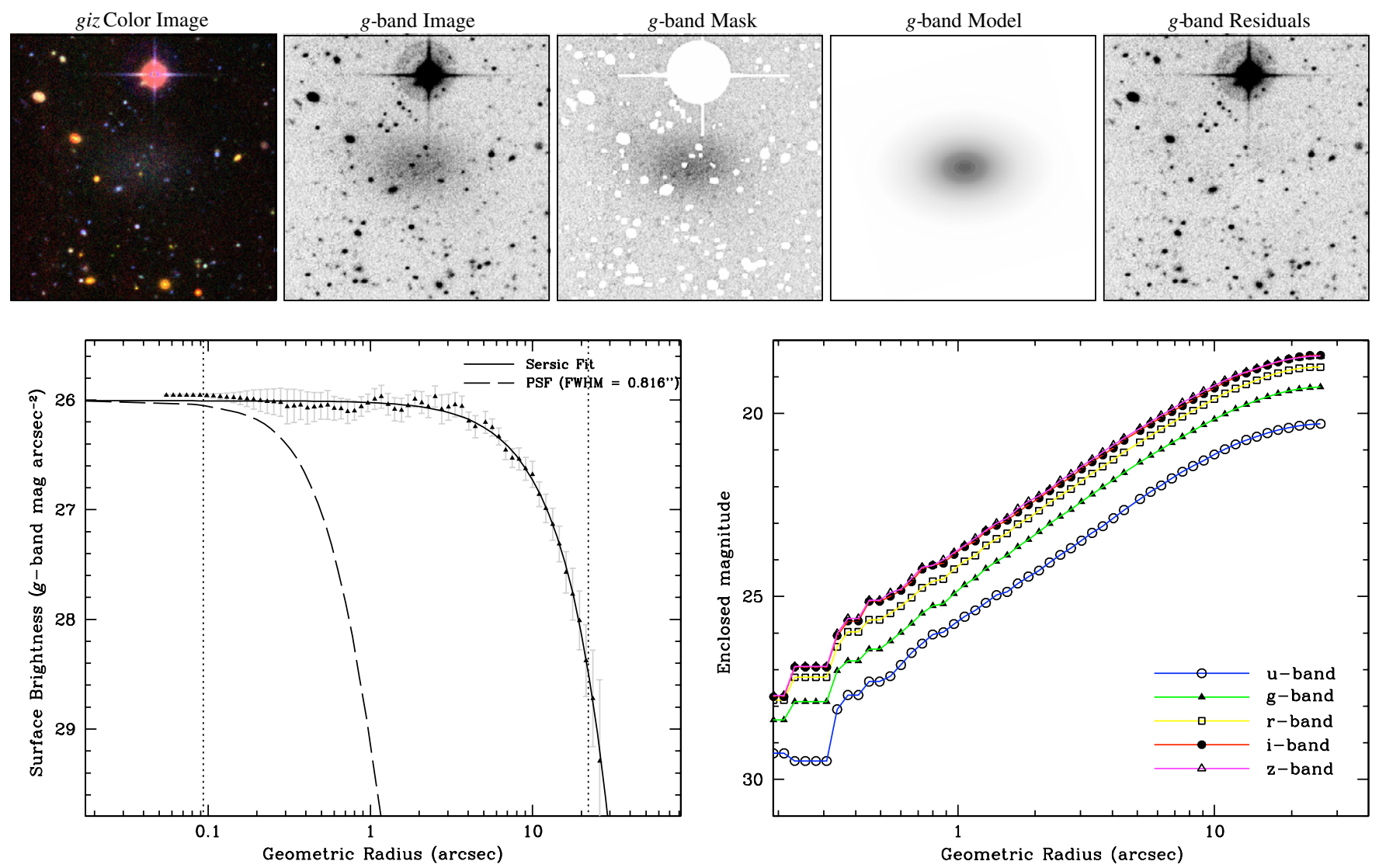

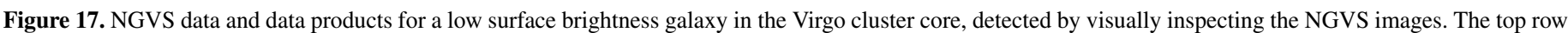

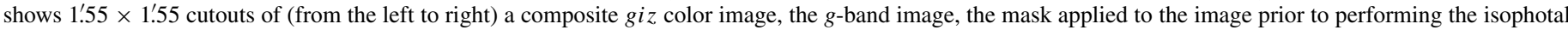

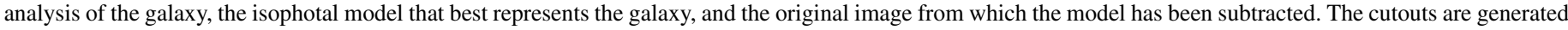

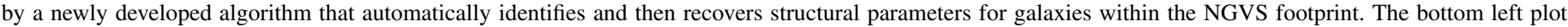

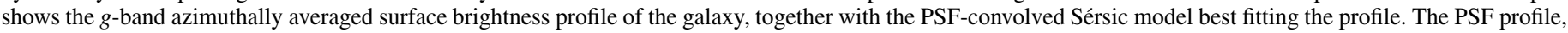

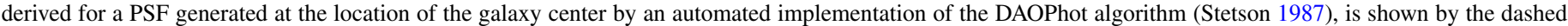
line. The bottom right panel shows non-parametric curves of growth in all five NGVS bands.

There is also evidence from many independent studies that suggests that there are important continuities in the photometric and structural properties of high-mass ("giant") and low-mass ("dwarf") galaxies (e.g., Jerjen \& Binggeli 1997; Graham et al. 2003; Graham \& Guzmán 2003; Gavazzi et al. 2005; Ferrarese et al. 2006a; Côté et al. 2006, 2007, 2008; Côté 2010; McLaughlin et al. 2012). For instance, Figure 16 shows photometric scaling relations for early-type galaxies in the Virgo and Fornax clusters (gray squares), taken mainly from the ACSVCS and its companion survey in Fornax (ACSFCS; Jordán et al. 2007), and from Gavazzi et al. (2005). To better define the faint end of the relations, early-type galaxies in the Local Group have been included (cyan squares). ${ }^{43}$ Smooth variations in effective radius and surface brightness with magnitude are reflected in the red curves (see McLaughlin et al. 2012), which give the expected relations for galaxies with Sérsic-like profiles whose density structure (i.e., departure from homology) varies smoothly along the luminosity function. At the bright end of the sample, $B \lesssim 12$, the galaxy sample shown in Figure 16 is essentially complete (for early-types only). However, as the upper panel shows, the completeness fraction falls dramatically at fainter magnitudes, reaching $\sim 25 \%$ at $B=16 \mathrm{mag}$ and $0 \%$ by $B=19.5$ mag.

\footnotetext{
43 Fornax and Local Group galaxies have been shifted to the distance of the Virgo cluster to highlight the expected form of the scaling relations in the NGVS.
}

The NGVS has been designed to provide a complete and unbiased characterization of the photometric and structural scaling relations of galaxies of all morphologies, from the brightest cluster members into the regime of the "dwarf" galaxies that dominate galaxy populations in terms of overall numbers. The dashed and dotted lines in Figure 16 compare the detection limits of the NGVS to those of the VCC, illustrating the improvements in resolution, depth, and surface brightness sensitivity (Section 5.1.1). Figure 17 shows a specific example that illustrates the ease with which galaxies at the faint end of the Virgo luminosity function, as currently defined, can be identified and characterized from NGVS images. The two leftmost panels in the upper row of Figure 17 show a composite giz color image and the $g$-band image, respectively, for the $g=19.3 \mathrm{mag}$ galaxy identified by the yellow star in Figure 16. The galaxy is one of $\sim 300$ galaxies that are not included in the VCC, and were identified by visually inspecting the NGVS images of the Virgo core. This particular galaxy was also detected in the deep CFHT $V$ and $I 3.75 \mathrm{deg}^{2}$ survey of the Virgo core recently described in Lieder et al. (2012); with a measured $V$-band effective surface brightness of $26.16 \mathrm{mag} \mathrm{arcsec}^{-2}$, it is one of the lowest surface brightness galaxies detected in that survey, but is well above the completeness limit of the NGVS data (see Figure 16). The rightmost three panels in the top row of Figure 17 show some of the products of a newly developed automated and iterative algorithm for the recovery of 


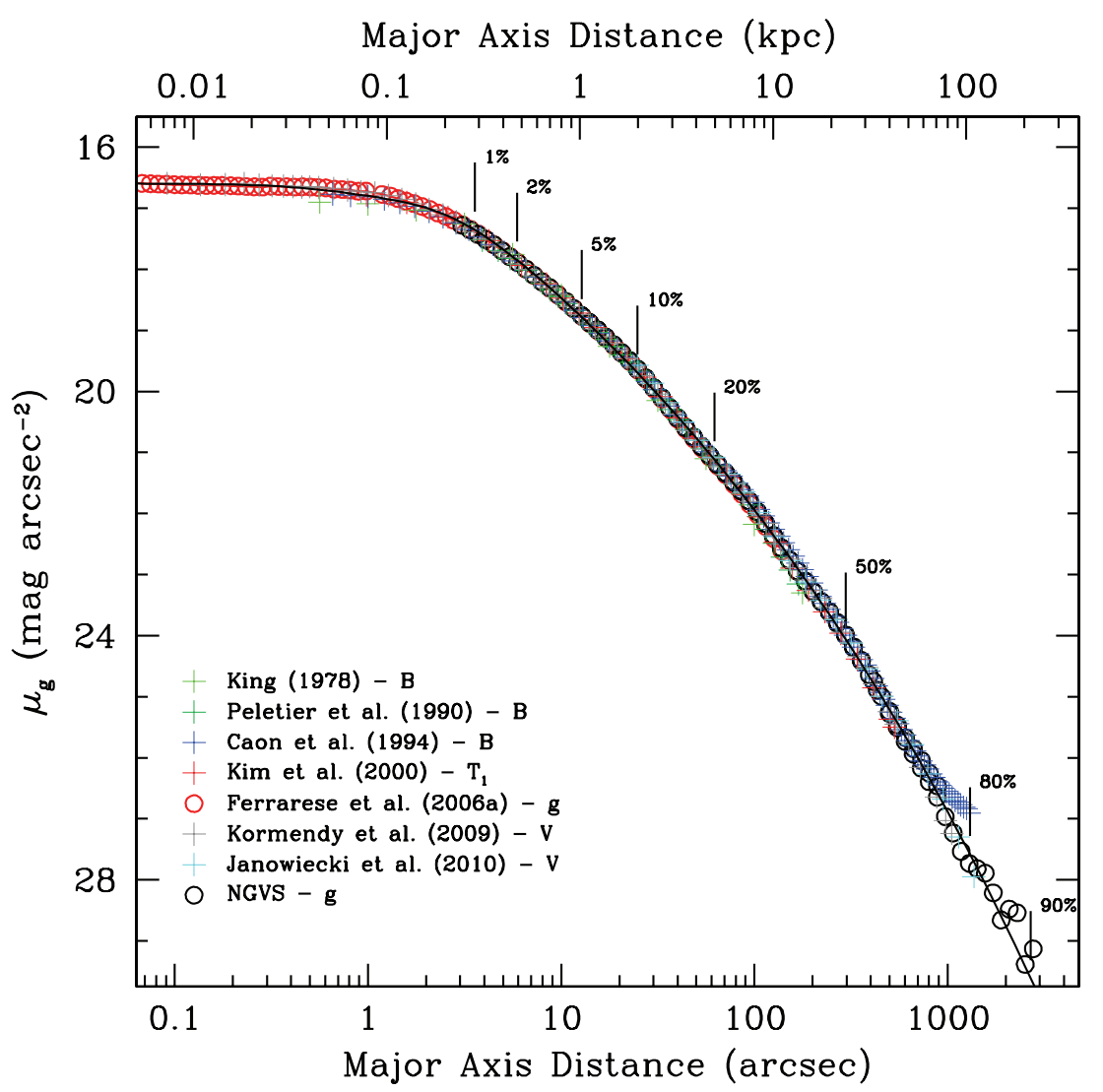

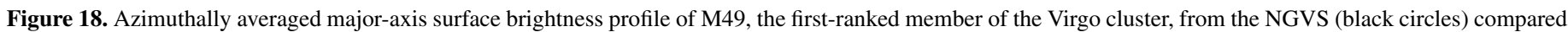

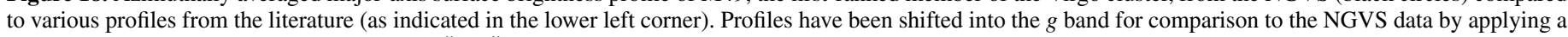

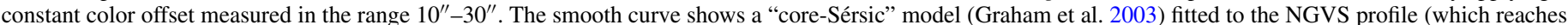

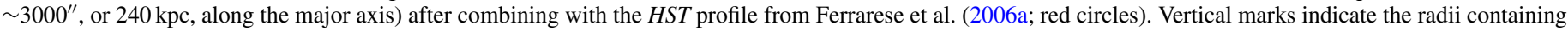
different percentages of the galaxy luminosity according to the fitted model.

galaxy structural parameters. Specifically, from left to right, we show the mask that is applied prior to performing the isophotal analysis, the model that, based on the isophotal analysis, best describes the galaxy, and the original image from which the model has been subtracted (the images show the results in the $g$ band, but similar products are generated for all available filters). The bottom left plot shows the $g$-band surface brightness profile derived from the isophotal analysis (a sky value, estimated during the fitting algorithm, is subtracted from the data), together with the PSF-convolved Sérsic model that best fits the profile. The bottom right panel shows a curve-of-growth analysis, in all bands. While the galaxy shown in Figure 17 is at the limit of current detections in the Virgo cluster, as Figure 16 shows, it will be possible to detect much fainter, low surface brightness galaxies down to $g \sim 25 \mathrm{mag}$. For all, it will be possible to measure the photometric and structural parameters from one-dimensional (1D) surface brightness profiles, 2D model fitting (Peng et al. 2002), and non-parametric curveof-growth methods (e.g., Janz \& Lisker 2008, 2009; Chen et al. 2010). In addition, it is important to note that it will be possible with NGVS imaging to resolve many compact systems in Virgo, allowing the identification of faint compact ellipticals (cEs) and UCDs. Although most (but not all; see Section 5.1.4) Virgo star clusters will appear unresolved in the NGVS, they are readily detectable as point sources to a typical depth of $g \approx 25.9$ mag.

At the same time, the large areal coverage will also allow an unprecedented characterization of the scaling relations for the brightest and most extended galaxies. Figure 18 shows a majoraxis surface brightness profile for VCC1226 (M49) based on NGVS $g$-band imaging; other profiles from the literature are shown for comparison. The NGVS profile itself extends from subarcsecond scales (although the data are plotted only for radii $\gtrsim 2^{\prime \prime}$ ) to $\simeq 2800^{\prime \prime}$, or roughly three times farther than previous state-of-the-art profiles. By combining the groundbased profile with that from HST/ACS (Ferrarese et al. 2006a), it is possible to define a composite profile that reaches inward to 0 '.05 (small squares). The solid curve in Figure 18 shows a coreSérsic model (Graham et al. 2003) fitted to this NGVS composite profile, allowing both the core and global properties to be derived with high precision. The left panel of Figure 19 shows a mosaic of four individual $g$-band MegaCam stacks, i.e., a $\sim 2 \times 2 \mathrm{deg}^{2}$ region $(\sim 570 \times 570 \mathrm{kpc}$, at a distance of $16.5 \mathrm{Mpc})$, in the core of Virgo's B subcluster. The red ellipses show the best-fit isophotes to M49 from which the surface brightness profile shown in Figure 18 was derived (although not shown here, contaminants, such as foreground stars, background objects, GCs, etc., were masked when fitting the isophotes). The right panel shows the residual image obtained by subtracting from the original image the model constructed from the ellipses that best fit the M49 light distribution; typical surface brightness levels in the diffuse structure seen in this image (which spans $149 \mathrm{kpc}$ on the side) are $\sim 27 \mathrm{mag} \operatorname{arcsec}^{-2}$. This example serves to illustrate the suitability of NGVS images in detecting shells and filaments that likely trace the past merging history of galaxies, as discussed in Section 5.1.8. 


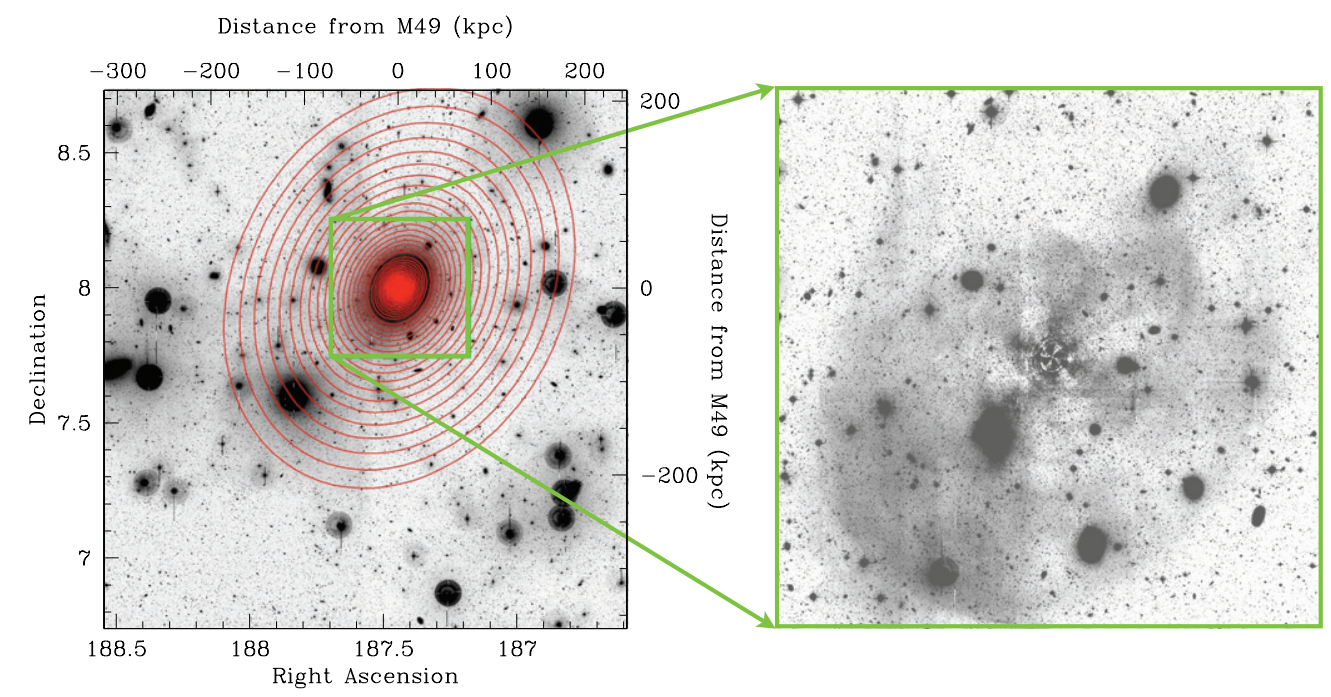

Figure 19. Left panel: a $2^{\circ} \times 2^{\circ} \mathrm{g}$-band image of the core of Virgo's B subcluster, from the NGVS. The lower and left axes show right ascension and declination, respectively, while the upper and right axes measure distance from the center of M49, in kpc (assuming a distance to Virgo of $16.5 \mathrm{Mpc}$ ). The red ellipses show the best-fit isophotes to M49; the surface brightness azimuthally averaged along these isophotes is shown in Figure 18 . Right panel: a $31^{\prime} \times 31^{\prime}(149 \times 149 \mathrm{kpc})$ region centered on M49 (i.e., the regions enclosed within the green box in the left panel), showing residuals obtained by subtracting from the actual image the model that best represents the azimuthally averaged elliptical isophotes. Note the extensive structure of shells and filaments. The central "wheel-like" residuals are indicative of higher-order components in the isophotal shapes compared to a simple ellipse.

(A color version of this figure is available in the online journal.)

Finally, we note that for a number of galaxies brighter than $B \sim 16$, long slit or Integral Field Unit (IFU) spectroscopy is also available (see Section 2.2 for a summary of past or ongoing spectroscopic surveys). In these cases, the NGVS photometric and structural parameters can be combined with rotation curves and velocity dispersion profiles, to examine the dynamical scaling relations (Tully-Fisher, Fundamental Plane, etc.) and their dependence on mass, morphology, and environment. The NGVS will allow an important first step toward defining a unified set of scaling relations to describe galaxies of different mass and morphology (e.g., Kassin et al. 2007; Dutton et al. 2010, 2011; McLaughlin et al. 2012).

\subsubsection{The Origin of Compact Stellar Systems}

The origin of compact, high surface brightness galaxies is a longstanding puzzle in the study of galaxy formation. The prototype of this class, M32, has been known to have unusual properties since the time of King (1962), who speculated that the galaxy's compact size (for its luminosity) was the result of tidal stripping by M31. Although similar conclusions were reached by Faber (1973a) and Keenan \& Innanen (1975), a rather different view introduced in the 1980s holds that cEs like M32 are actually the low-luminosity analogs of "giant" E galaxies, and hence, share with them a common formation mechanism (Wirth \& Gallagher 1984; Kormendy 1985; Kormendy et al. 2009). A number of recent investigations into the photometric and structural properties of early-type galaxies in the local universe have reached a conclusion more in line with the original view, i.e., that $\mathrm{dE} / \mathrm{dSO}$ galaxies represent the true low-luminosity extension of the "giant" E/SO sequences and that $\mathrm{cE}$ galaxies are anomalous systems that owe their unusual properties to tidal effects. Indeed, recent analytical calculations and numerical simulations suggest that tidal interactions have likely played a part in producing the unusual properties of M32 in particular, and of cEs in general (e.g., Bekki et al. 2001; Block et al. 2006; Chilingarian et al. 2009; McLaughlin et al. 2012).
In their study of structural parameters for dynamically hot galaxies, Bender et al. (1992) found that only one object (NGC5845) out of $\sim 350$ galaxies in their sample showed a size similar to those of the three cEs that define the class (M32, NGC4486B, and NGC5846A). As each of these cEs was found to lie close to a much more massive galaxy, the authors also suggested that tidal stripping may play a role in their formation. Systematic searches have subsequently uncovered a number of additional cE galaxies (e.g., Smith Castelli et al. 2008; Chilingarian et al. 2009; Huxor et al. 2011), all of which are also found close to massive galaxies. In a few cases, significant tidal interactions with the massive galaxy are clearly underway - prima facie evidence that tidal stripping must play a role in the formation of at least some cEs. Of course, it remains a possibility that more than a single formation channel can lead to objects with cE-like properties.

A development over the past decade that may hold a new clue to the origin of cEs is the discovery of numerous UCD galaxies in cluster environments (e.g., Hilker et al. 1999; Drinkwater et al. 2000, 2003; Phillipps et al. 2001; Haşegan et al. 2005; Jones et al. 2006; Blakeslee \& Barber DeGraaff 2008; Madrid et al. 2010; Chiboucas et al. 2011). Collectively, the scaling relations of GCs and UCDs appear to show a "break" at $\approx 2 \times 10^{6}$ solar masses (Haşegan et al. 2005; Mieske et al. 2008), suggesting that UCDs may represent a distinct population of compact stellar systems that are intermediate in properties between GCs and cEs (Haşegan et al. 2005; Dabringhausen et al. 2008). Leading theories for the formation of UCDs include hierarchical merging of stellar clusters, possibly in actively starforming environments (e.g., Fellhauer \& Kroupa 2002) and tidal stripping of nucleated galaxies (e.g., Bekki et al. 2003; see also Bassino et al. 1994). In fact, there is evidence that each of these processes plays a role in the formation of individual UCDs, i.e., the young massive star cluster W3 in NGC 7252 may be a UCD progenitor that has formed through successive mergers of star clusters (Fellhauer \& Kroupa 2005), while a few UCDs in the Virgo and Fornax clusters exhibit a diffuse envelope surrounding 


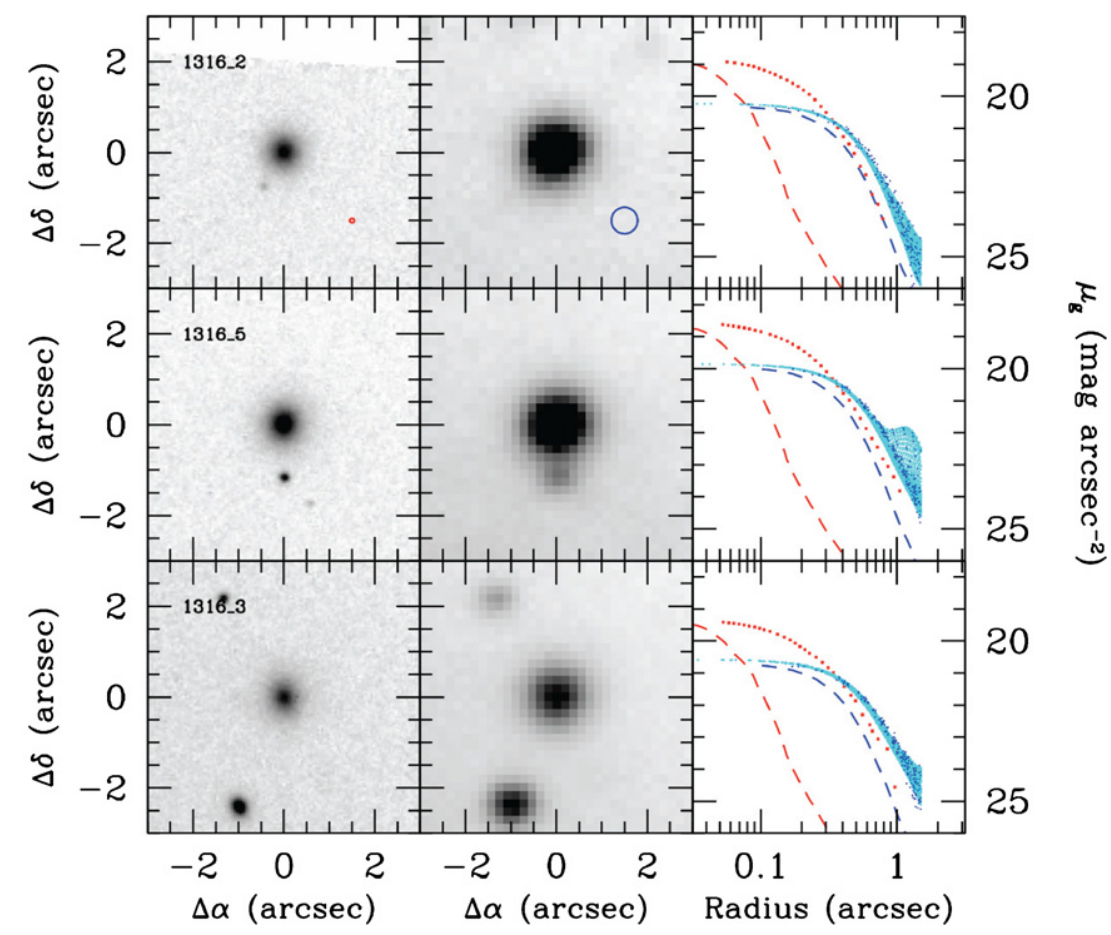

Figure 20. Images and 1D surface brightness profiles for three spectroscopically confirmed UCDs in the core of the Virgo cluster $\left(1316 \_2=\right.$ S999; 1316_5 = S928; and 1316_3 = H8005; see Haşegan et al. 2005). Along each row, we show the HST F475W image (6" $\left.6^{\prime \prime} 6^{\prime \prime}\right)$ from the ACS Virgo Cluster Survey (left panel), the $g$-band image from the NGVS (middle panel) and the surface brightness profiles derived from these images (right panel). The red and blue circles in the first two panels along the upper row compare the seeing disks from $H S T$ (FWHM $\left.\simeq 00^{\prime \prime} 1\right)$ and CFHT $\left(\simeq 0^{\prime \prime} 58\right)$. The corresponding PSF profiles are plotted as the red and blue dashed curves, respectively, in the right panels. The red and blue points show, respectively, the $H S T$ and NGVS profiles for each UCD, while the cyan dots show the expected pixel values for the NGVS imaging after convolving the best-fit UCD model derived from the HST imaging with the NGVS PSF (note that neighboring objects are not masked, explaining the flaring in the cyan points in the middle right panel). This comparison demonstrates that these UCDs, which have half-light radii in the range $r_{h} \approx 20-25 \mathrm{pc}$, are spatially resolved in the NGVS imaging.

the compact "nucleus" (e.g., Haşegan et al. 2005; Evstigneeva et al. 2007; Chilingarian \& Mamon 2008), as would be expected in stripping models.

The viability of the different formation channels proposed for cEs and UCDs-and the possible connections between these compact stellar systems-remain open questions. Unfortunately, reliable constraints on the overall numbers (and physical properties) of cEs and UCDs based on volume- and/or luminosity-limited samples are lacking, with the result that conclusions must presently be drawn from samples that are collected from a variety of sources, often with different selection functions and observational biases. One of the goals of the NGVS is to carry out a detailed census of compact stellar systems in a common environment using deep and homogeneous imaging. The traditional challenges facing such efforts have been limited areal coverage, depth, and angular resolution. Consider the survey of Binggeli et al. (1985), which was partly motivated by the search for compact galaxies in Virgo (see their Table XIII for a list of M32-type candidates). Their seeing (median FWHM $\sim 1^{\prime \prime} .25$, corresponding to $100 \mathrm{pc}$ at the distance of Virgo; Binggeli et al. $1984)$ and relatively bright limiting magnitude $\left(B_{\lim } \sim 20 \mathrm{mag}\right.$ for galaxies and $\sim 23$ for unresolved sources; Binggeli et al. 1985 ) would have precluded the detection of compact and/or low-luminosity systems in the range $M_{B} \gtrsim-13$ to $-11 \mathrm{mag}$, meaning that faint cEs and/or bright UCDs could have gone undetected.

By contrast, the $i$-band seeing constraint of FWHM $\leqslant 0$ '. 6 for the NGVS and the survey's $10 \sigma$ (point-source) limiting magnitude of $g=25.9 \mathrm{mag}$, correspond to, respectively, $\sim 50 \mathrm{pc}$ and $M_{g} \sim-5.3 \mathrm{mag}$, or $M_{B} \sim-5.0 \mathrm{mag}$. Figure 20 illustrates the effectiveness of NGVS for identifying compact stellar sources by showing a comparison of the $g$-band images from the NGVS to those from $H S T$ /ACS for three spectroscopically confirmed UCDs in the cluster core (Haşegan et al. 2005). Although the superior angular resolution of $H S T$ is apparent (i.e., an FWHM $\approx 0$ "' 1 compared with $\approx 0$ '.58 for the CFHT images), the third column of this figure shows from a comparison of the 1D surface brightness profiles that these UCDs-which have effective radii in the range $20 \mathrm{pc} \lesssim r_{e} \lesssim 25 \mathrm{pc}$ and are thus representative of most known UCDs-are readily resolved in the CFHT imaging. We can quantify this result by comparing the NGVS imaging for UCDs and GCs identified in the ACSVCS (Jordán et al. 2009), which suggests that NGVS imaging should enable us to identify compact stellar sources in the Virgo cluster with effective radii as small as $r_{e} \sim 10 \mathrm{pc}$.

\subsubsection{Galaxy Centers: Stellar Nuclei and Supermassive Black Holes}

The innermost regions of galaxies are of special importance in galaxy formation, as they contain a fossil record of the complex processes by which the galaxies formed and evolved. Because dynamical timescales in the cores are shorter than those elsewhere in the galaxy, the morphology, dynamics, and stellar populations in these regions are imprinted with signatures of the gas, dust, and stellar systems (e.g., low-mass galaxies, star clusters) that were drawn to the bottom of the gravitational potential well over cosmic timescales. Careful observations made over the last few decades, particularly with HST, have shown that many (and possibly all) high- and intermediate-mass galaxies contain central supermassive black holes (SMBHs). The properties of these SMBHs are in turn linked to those of their host galaxies 
through a variety of scaling relations (see, e.g., the review of Ferrarese \& Ford 2005) that are generally interpreted as prima facie evidence for the importance of nuclear inflows and AGN feedback in galaxy formation. For example, it is now suspected that AGN processes play an important role in explaining some longstanding puzzles in galaxy formation, including the exponential cutoff at the bright end of the galaxy luminosity function and the bimodal separation of galaxies into red and blue sequences (e.g., Croton et al. 2006; Bower et al. 2006; see also Section 5.1.2)

An unexpected result to have emerged from HST imaging surveys of nearby galaxies during the past decade, particularly the ACSVCS and ACSFCS (Côté et al. 2004, 2007; Jordán et al. 2007), is the discovery that most intermediate- and low-mass galaxies contain structurally distinct stellar components in their cores. ${ }^{44}$ This is true for galaxies across the Hubble sequence (Carollo et al. 1998; Böker et al. 2002, 2004; Walcher et al. 2005, 2006; Seth et al. 2008) and along the luminosity function (e.g., Côté et al. 2006; Turner et al. 2012), suggesting that a very generic formation process (or processes) is needed to explain these distinct stellar nuclei. Moreover, a possible connection of the nuclei to SMBHs is suggested by the discovery that stellar nuclei in low-mass galaxies make up roughly the same fraction of galaxy (baryonic) mass, $\eta \sim 0.2 \%$, as the SMBHs in massive galaxies (Côté et al. 2006; Ferrarese et al. 2006b; Wehner \& Harris 2006; Rossa et al. 2006).

Such a nucleus-SMBH connection could arise in a number of ways. For instance, McLaughlin et al. (2006) have suggested that momentum feedback from super-Eddington accretion in high-mass galaxies, and from stellar winds and supernovae in low-mass galaxies, could lead to the observed relation. $\mathrm{Li}$ et al. (2007) argued that the observed relation may be a consequence of gas depletion via accretion and star formation, while Hopkins et al. (2009) have shown that star formation triggered by gas inflows during mergers would lead to embedded stellar components with properties similar to those observed in the more massive galaxies. Alternatively, Merritt \& Szell (2006) have argued that, in low-mass galaxies, stellar density cusps can grow around central black holes via energy exchanges. The growth of a central massive object in the centers of most galaxies would also be expected in models where star clusters repeatedly spiral inward as a result of dynamical friction (e.g., CapuzzoDolcetta \& Miocchi 2008; Capuzzo-Dolcetta \& MastrobuonoBattisti 2009). Of course, there is no reason for these processes to be mutually exclusive, and it is reasonable to expect that their relative efficiencies vary smoothly with galaxy properties and local environment (Côté et al. 2007).

Although the NGVS image quality is $\sim 5-7 \times$ poorer than that of HST in terms of FWHM (Section 3), there are several important ways in which NGVS significantly extends the HST's ACSVCS study of stellar nuclei. First, Figure 21 shows azimuthally averaged surface brightness profiles for a number of low- and intermediate-luminosity Virgo galaxies, including four that appeared in the ACSVCS (Ferrarese et al. 2006a; Côté et al. 2006). This comparison shows that although actually resolving the nuclei is challenging or impossible with the NGVS imaging, it is nevertheless a straightforward exercise to reliably identify

\footnotetext{
44 Different terminology has been used to denote these components, including "compact stellar nuclei," "nuclear clusters," or "extra light." In most cases, however, the operational definition of the central components is the same: a central luminosity excess relative to the inward extrapolation of a model, usually a Sérsic model, fitted to the light distribution of the outer galaxy; see Section 3 and Appendix A of Côté et al. (2006).
}

the nuclei in low- and intermediate-luminosity galaxies, and measure their luminosities and colors with good accuracy (the nucleus-to-galaxy luminosity fraction, $\eta$, from HST and NGVS data, is given in each panel). Second, the areal coverage of the NGVS allows the large-scale global profiles of galaxies to be measured with high precision (see, e.g., Figure 18), thus greatly aiding the nucleus-galaxy decomposition. Finally, and most importantly, the ACSVCS studied only 100 Virgo cluster galaxies, all brighter than $g \sim 15.7 \mathrm{mag}$ and with earlytype morphologies. In the NGVS, it will therefore be possible to extend the study of nuclei (e.g., frequency of nucleation, luminosity functions, mass fractions, CMRs, etc.) to galaxies hundreds of times fainter than the lowest-luminosity galaxies in the ACSVCS, and to explore possible dependencies of nuclear properties on morphology and environment (e.g., Ferguson \& Sandage 1989; Binggeli et al. 1987; Côté et al. 2006; Lisker et al. 2007).

Comprehensive follow-up of NGVS galaxies (both nucleated and non-nucleated) at X-ray, mid-IR, and radio wavelengths will make it possible to explore further the relationship between nuclei and SMBHs (e.g., Decarli et al. 2007; Seth et al. 2008; see also Volonteri et al. 2008), building upon what has been done to date for ACSVCS galaxies (e.g., Gallo et al. 2008, 2010; Capetti et al. 2009).

\subsubsection{Extragalactic Star Clusters}

Nearly all galaxies brighter than a few hundred million solar luminosities contain a population of star clusters. As the best approximations found in nature to simple stellar populations (SSPs), such star clusters provide both important probes of galaxy formation and test beds for stellar dynamical and evolutionary models (see, e.g., the reviews of Harris 2001; West et al. 2004; Brodie \& Strader 2006; Portegies Zwart et al. 2010).

The power of high-quality, homogeneous imaging for star clusters in large samples of galaxies was demonstrated by early archival programs with HST (Gebhardt \& Kissler-Patig 1999; Kundu \& Whitmore 2001; Larsen et al. 2001). Later, these studies were greatly extended in scope by the ACSVCS and ACSFCS - programs that were designed from the outset to study the systems of (globular) clusters associated with early-type galaxies in the Virgo and Fornax clusters (Côté et al. 2004; Jordán et al. 2007). HST imaging for many thousands of old star clusters belonging to these galaxies was used to examine their basic properties in relation to those of their host galaxies (e.g., Peng et al. 2006a, 2006b, 2008; Mieske et al. 2006, 2010; Jordán et al. 2004, 2005, 2007, 2009; Sivakoff et al. 2007; Villegas et al. 2010; Masters et al. 2010; Liu et al. 2011). The key advantages of HST in the study of extragalactic star clusters are its photometric precision and angular resolution, allowing most clusters, which have $\left\langle r_{h}\right\rangle \sim 3 \mathrm{pc}$, to be spatially resolved at the distance of the Virgo cluster. ${ }^{45}$

At the same time, there are a number of open questions concerning extragalactic star clusters that are best addressed with deep, multi-color, wide-field imaging such as that available from the NGVS. To illustrate this, Figure 22 shows a variety of color distributions for nearly $10,000 \mathrm{GC}$ candidates within $1^{\circ}$ of M87, identified in the NGVS images on the basis of magnitude ( $20 \leqslant g \leqslant 24)$, ellipticity $(\epsilon \leqslant 0.2)$, light concentration, and colors. The color selection was based on

\footnotetext{
45 Two-dimensional modeling of confirmed Virgo star clusters in the NGVS imaging suggests that effective radii for star clusters brighter than $i \approx 23$ can be resolved down to a size of $r_{h} \sim 10 \mathrm{pc}$; see Section 5.1.4.
} 


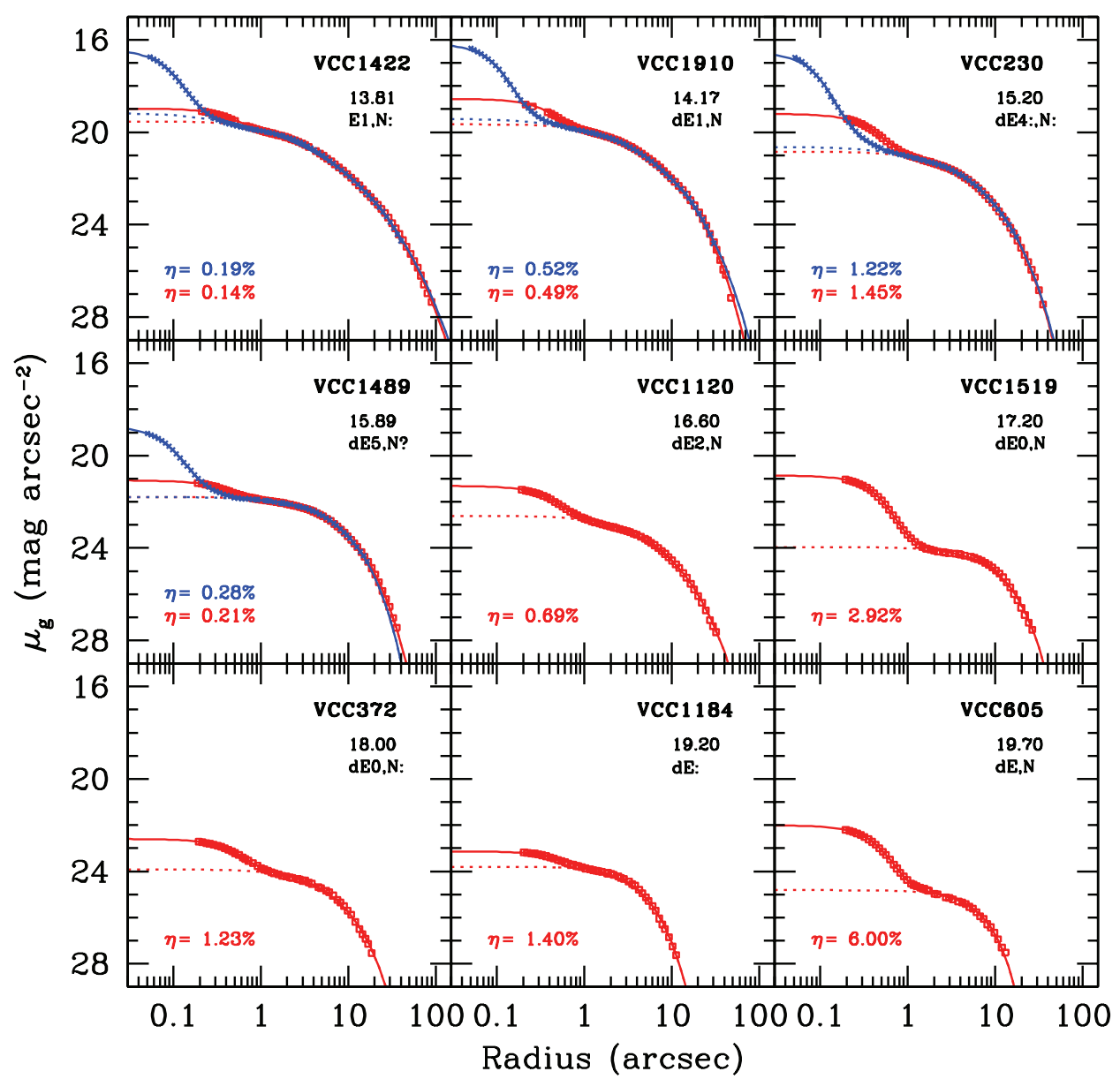

Figure 21. Stellar nuclei in intermediate- and low-mass Virgo cluster galaxies. The nine objects shown here are representative of the early-type galaxy population in Virgo and span a factor of $\sim 230$ in luminosity. In each panel, the red squares show the azimuthally averaged $g$-band surface brightness profile from the NGVS. As the four brightest galaxies also appear in the HST ACSVCS (Ferrarese et al. 2006a; Côté et al. 2006), we also plotted the HST/ACS profile (blue crosses) after applying a $0.1 \mathrm{mag}$ correction to the $H S T$ profiles (see the text for details). For both data sets, the full curves show the double-Sérsic model that best fits the observed nucleus/galaxy profile; the dotted curves show the single-Sérsic component fitted to the galaxy. The nucleus-to-galaxy luminosity ratio, $\eta$, determined in the ACSVCS (blue) and NGVS (red) is given in the lower left of each panel. The information in the upper right corner of each panel is taken directly from Binggeli et al. (1985); a ":" denotes uncertain classification.

$(u-g)-(g-i)$ and $(g-i)-(i-z)$ density maps constructed using a sample of 756 velocity-confirmed GCs in M87 (selected from NGVS images and follow-up spectroscopy, and from the compilation of Hanes et al. 2001) and M31 (Peacock et al. 2010): GCs were included in Figure 22 if their location in both $(u-g)-(g-i)$ and $(g-i)-(i-z)$ color-color diagrams fell within the smallest density contour including all spectroscopically confirmed clusters.

This example demonstrates that it will be possible with the NGVS to carry out a comprehensive study of the largescale distribution of star clusters within galaxy halos. This is a fundamental property of cluster systems that remains very poorly constrained at present, with much of our current understanding hinging on imaging for a handful of galaxies (e.g., Harris 1986; Geisler et al. 1996; Rhode \& Zepf 2004; Tamura et al. 2006; Harris 2009). Likewise, the NGVS can be used to map the distribution of stars clusters not only within galaxy halos, but also on intergalactic scales, where they are expected to trace the ICL component (e.g., West et al. 1995; Jordán et al. 2003; Lee et al. 2010; see also Section 5.1.8).

Additional issues that will be explored with the NGVS include the efficiency of star cluster formation as a function of mass, morphology, and local environment; the color-metallicity transformation of old star clusters and its dependence on color index (Yoon et al. 2006; Cantiello \& Blakeslee 2007); the role of self-enrichment in explaining the GC CMR, i.e., the "blue tilt" (Strader et al. 2006; Harris et al. 2006; Peng et al. 2009); the shape of the cluster luminosity/mass function across galaxies spanning a range in mean age and morphology; and the relation of star clusters to UCDs from photometric, dynamical, and structural scaling relations (see Figure 10 of Haşegan et al. 2005; Section 5.1.4). Above all, the NGVS will provide a unique opportunity to examine homogeneously the basic properties of star clusters in galaxies spanning a wide range in mass, morphology, and environment.

Finally, we mention here that several follow-up programs, ranging form NIR imaging to optical spectroscopy, have been undertaken to capitalize on the census of Virgo globular clusters provided by the NGVS. These programs are briefly described in Section 6.

\subsubsection{Extragalactic Distance Scale and the Structure of the Virgo Cluster}

Virgo is a large, irregularly shaped cluster that is continuing to accrete galaxies from the extended supercluster in which it is embedded (Section 2): as such, it is an excellent laboratory for studying the connections between galaxy properties and local environment (see especially Section 5.1.9). Virgo's proximity 

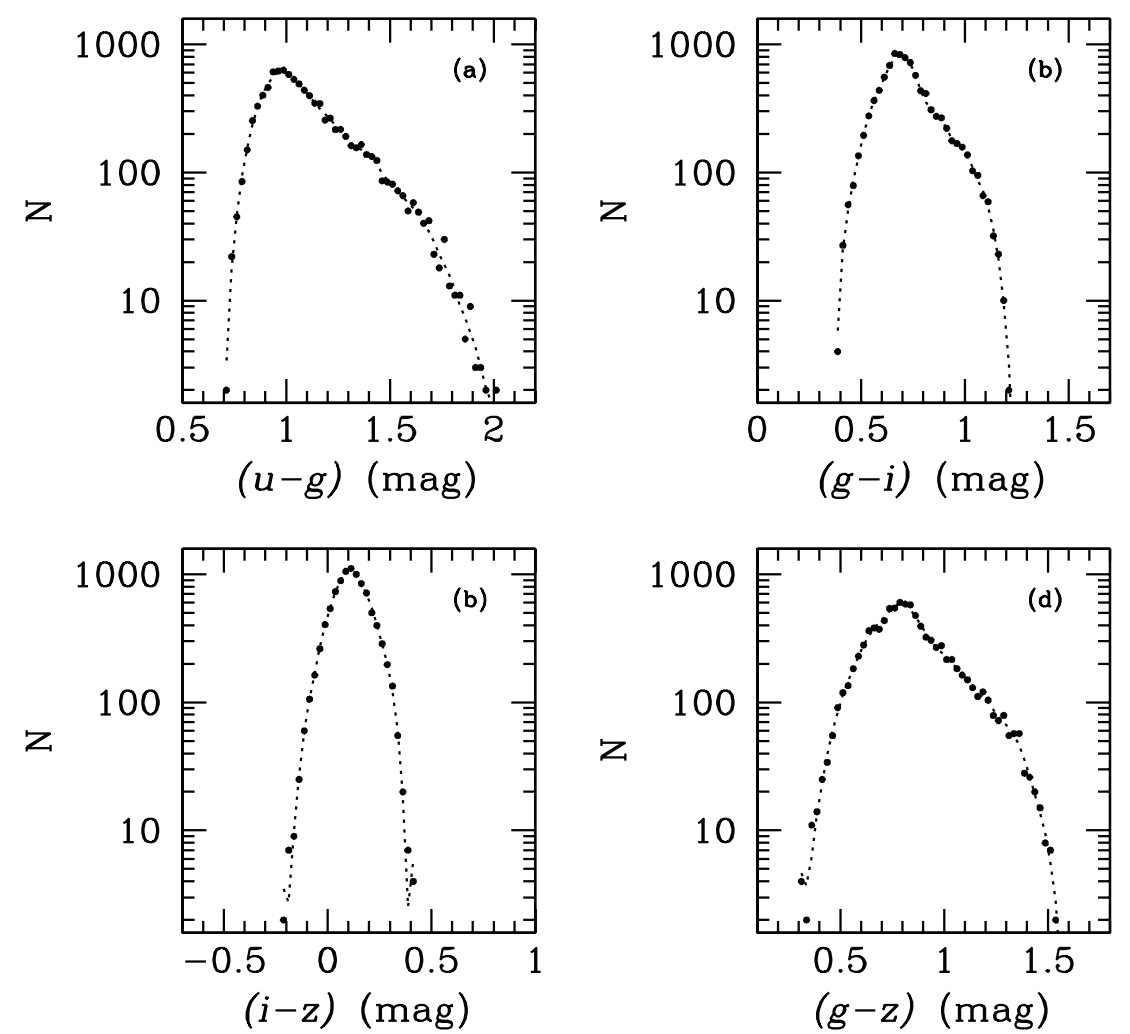

Figure 22. Extinction-corrected color distributions for GC candidates in the core of the Virgo cluster. Histograms are shown in a variety of color indices: $(u-g)$, $(g-i),(i-z)$, and $(g-z)$. These distributions are based on a sample of $9522 \mathrm{GC}$ candidates located with $1^{\circ}$ of M87 and selected on the basis of magnitude $(20 \leqslant g \leqslant 24)$, ellipticity $(\epsilon \leqslant 0.2)$, light concentration, and location in the $(u-g)-(g-i)$ and $(g-i)-(i-z)$ color-color diagrams. The dotted curves show a (non-parametric) locally weighted scatter plot smoothing (LOWESS) fit to the data (see Cleveland \& McGill 1984).

is an important advantage for such studies: at a mean distance of $16.5 \mathrm{Mpc}$, it is possible to recover the true three-dimensional (3D) galaxy distribution using individual distances determined by the SBF method (Tonry \& Schneider 1988; see also West \& Blakeslee 2000; Neilsen \& Tsvetanov 2000; Jerjen et al. 2004), at least for early-type galaxies and dust-free bulges of spirals. High-precision SBF distances were measured as part of the ACSVCS for 92 galaxies classified as cluster members in the VCC (Mei et al. 2007; Blakeslee et al. 2009, 2010). This study revealed the back-to-front $(2 \sigma)$ depth of the dominant early-type population to be $2.4 \pm 0.4 \mathrm{Mpc}$, although there is at least one sizable group of early-type galaxies projected among the cluster galaxies and having the same recessional velocity, but with a significantly larger distance, i.e., the $\mathrm{W}^{\prime}$ group at $\approx 23 \mathrm{Mpc}$. Parallel efforts using the Tully-Fisher relation indicate that the later-type Virgo galaxies are spread out over a considerable range in distance (Yasuda et al. 1997; Gavazzi et al. 1999).

The ability to measure the SBF signal and correct for contamination (e.g., faint background galaxies, GCs, etc.) depends on the $\mathrm{S} / \mathrm{N}$ and resolution of the images. Recent SBF measurements with $H S T /$ ACS have an accuracy of $~ 4 \%$ (Mei et al. 2007; Cantiello et al. 2007; Blakeslee et al. 2009, 2010), or about $0.7 \mathrm{Mpc}$ at the distance of Virgo; this is sufficient for resolving the cluster depth. In comparison, the distance uncertainties from the SBF measurements of Tonry et al. (2001), which were obtained under ground-based conditions with seeing $\gtrsim 1^{\prime \prime}$ and small-format CCD detectors, are $\sim 2.5 \times$ larger, or about $1.7 \mathrm{Mpc}$ for Virgo, precluding a clear resolution of the cluster's line-of-sight structure.
In several key respects, the NGVS is more similar to the $H S T /$ ACS studies than to the (heterogeneous) ground-based program of Tonry et al. (2001). For instance, the NGVS data are all taken with a single telescope/instrument combination, under tightly controlled photometric and image quality constraints. The $i$-band seeing of $\lesssim 0^{\prime}$. 6 FWHM means that the resolution of the NGVS images is approximately midway between the ACS and earlier ground-based data. The depth of the NGVS images also allows us to reach well beyond the peak of the GC luminosity function (Section 5.1.6), greatly reducing their contamination to the SBF signal compared with previous ground-based studies.

Moreover, the multi-band coverage of the NGVS will provide better stellar population information than was available for any previous SBF study. The absolute SBF magnitude is usually calibrated based on a single photometric color, but since age and metallicity are not fully degenerate in their effects on SBF magnitude and optical colors, stellar population variations at a given integrated color can cause significant calibration uncertainties. This is especially true for bluer galaxies, $(g-z) \lesssim 1$, which are a mix of young and metalpoor systems, and may have SBF calibration uncertainties of $\sim 0.1$ mag or more. The multi-band photometry of NGVS, which includes the $(u-g)$ color index that is useful for characterizing the $4000 \AA$ break, will help ensure accurate calibration of the measured $i$-band SBF magnitudes as a multivariate function of stellar population, thereby reducing the final error in distance.

Based on previous SBF work under the very best groundbased conditions, we anticipate distance errors of $0.13 \mathrm{mag}$ 
from the NGVS data, corresponding to $\approx 1 \mathrm{Mpc}$; preliminary analysis of the NGVS data indeed supports this estimate. As this level of precision is adequate for distinguishing between galaxies in the core, outskirts, or separate structures projected along the line of sight, it will be possible to study how galaxy properties correlate with the true local environment determined from 3D positions. The resulting sample of SBF distances will be the largest available for the Virgo cluster: Combined with the existing ACSVCS measurements, which can reach lower galaxy luminosities but lack the wide-field coverage of NGVS, we expect to obtain distances for more than 200 galaxies. Besides information on the local environments of individual galaxies, these data will enable improved constraints on the 3D shape of Virgo, substructures, infall, and spatial segregation by galaxy type.

\subsubsection{Diffuse Intracluster Light}

The diffuse ICL has emerged as an important tool for studying the evolution of galaxy clusters. This component is an inevitable result of the gravitational stripping of stars from galaxies during cluster assembly and evolution (e.g., Dubinski 1998; Murante et al. 2004, 2007; Willman et al. 2004; Sommer-Larsen et al. 2005; Rudick et al. 2006, 2009; Guo et al. 2011). However, the dominant physical mechanism driving the ICL production remains unclear. Stripping processes in clusters are numerous and often operate simultaneously: Mergers that grow the central galaxy (Murante et al. 2007; Conroy et al. 2007), accretion of infalling groups (Mihos 2004; Willman et al. 2004; Rudick et al. 2006), harassment of low-luminosity galaxies (Moore et al. 1996), and slow tidal stripping of orbiting galaxies (see Purcell et al. 2007, and references therein) may all contribute to the formation of the ICL. The amount of ICL grows with time as the cluster assembles (Willman et al. 2004; Rudick et al. 2006; Murante et al. 2007), and a significant amount of this diffuse light first takes the form of kinematically coherent streams that are later disrupted and mixed into a more diffuse ICL (Rudick et al. 2009). Generally speaking, the morphological and kinematic properties of the ICL on large scales $(\sim 100 \mathrm{kpc})$ contain information on the dynamical state of the cluster, while on smaller scales $(\sim 10 \mathrm{kpc})$ the presence of tidal debris around individual galaxies is a signature of strong interactions between galaxies or with the cluster tidal field itself.

The Virgo cluster is a particularly useful environment for studying ICL. The spatial, kinematic, and morphological substructure in Virgo (Sections 2 and 5.1.7) argues for a dynamically active cluster, where the production of an ICL component is ongoing. The cluster is well studied at a variety of wavelengths, giving us a comprehensive view of it, and its member galaxies, at high spatial resolution. Its proximity also permits the study of discrete tracers of the ICL, such as intracluster red giant stars, planetary nebulae (Arnaboldi et al. 2002; Arnaboldi 2005; Feldmeier et al. 2003, 2004; Aguerri et al. 2005), and GCs (West et al. 1995, 2011; Jordán et al. 2003).

Tidal streamers and fine structure around Virgo galaxies were first detailed in deep photographic work by Malin (1979, 1994); subsequent deep imaging by Mihos et al. (2005, 2009) and Rudick et al. (2010) has revealed a complex web of ICL permeating the Virgo cluster core, as well as a number of tidal streams and shells surrounding individual Virgo ellipticals (Janowiecki et al. 2010) tracing the accretion history of these galaxies. Intracluster red giant stars have been found via deep HST imaging of Virgo (Ferguson et al. 1998; Durrell et al. 2002; Williams et al. 2007a); the CMD of these stars argues for an old, metal-poor ICL population (Williams et al. 2007a), consistent with the color of the Virgo ICL streamers measured by Rudick et al. (2010). A number of intracluster GCs have also been found in Virgo (Williams et al. 2007b), and Lee et al. (2010) have used SDSS imaging to color-select GC candidates and trace out their spatial distribution across Virgo (see also Section 5.2.2).

The NGVS will expand the study of Virgo's ICL in a number of ways. In terms of deep surface photometry, NGVS can probe down to $\mu_{g} \sim 29 \mathrm{mag} \operatorname{arcsec}^{-2}$ (see Figures 18 and 19) over large angular scales, and recover many of the faint ICL features identified in the even deeper Schmidt survey of Mihos et al. (2005; see Figure 23). However, the areal coverage of the NGVS is much larger than that of the Schmidt survey $\left(104 \mathrm{deg}^{2}\right.$ versus $16 \mathrm{deg}^{2}$ ), allowing the ICL to be mapped over the entire extent of the Virgo cluster. This is particularly important, as the stripping processes in the outskirts of Virgo may well be different from those dominating deep inside the cluster core-differences in the amount of ICL and its morphological structure will help discriminate between the various processes for ICL formation. Moreover, with multiband NGVS photometry, we can measure ICL colors and place stronger constraints on the stellar populations and production mechanisms for the ICL. For example, if there is a significant component of the ICL formed during mergers of massive galaxies (e.g., Murante et al. 2007), the ICL colors should be quite red, reflecting a more metal-rich population of stars. Conversely, any ICL formed from star-forming gas recently stripped from cluster galaxies should be preferentially younger and bluer. While contamination due to backscattered light from Milky Way dust complicates the interpretation of the diffuse light in Virgo (Rudick et al. 2010; Cortese et al. 2010), this Galactic dust is effectively traced in the ultraviolet, infrared, and in $\mathrm{H}$ I emission, allowing for some differentiation between true ICL features and Galactic dust using deep GALEX (Boselli et al. 2011), Herschel (Davies et al. 2010, 2012), and Arecibo (Giovanelli et al. 2005; Haynes et al. 2011) surveys of the Virgo cluster.

GCs detected in the NGVS (see Section 5.1.6) provide a second, independent probe of Virgo's ICL, as the most remote GCs likely represent discrete tracers of the ICL. NGVS multicolor imaging efficiently separates Virgo GCs from background contaminants down to $i \sim 24 \mathrm{mag}, 2$ mag fainter than the Virgowide survey for GC candidates using SDSS imaging by Lee et al. (2010). This huge gain in depth will allow the ICL to be mapped with GCs to very low densities - far below what can be achieved through surface photometry. Simulations show that much of the kinematic history of the ICL is contained in unmixed material with extremely low surface brightness (Willman et al. 2004; Rudick et al. 2009), and follow-up spectroscopy of the brighter GC candidates (e.g., Côté et al. 2001, 2003; Hwang et al. 2008) will provide the velocities needed to identify ICL substructures, characterize the fraction of ICL in cold streams, and separate bonafide intracluster GCs from bound populations.

\subsubsection{Galaxy Interactions and Evolution: The Role of Environment}

Galaxy properties and evolution are influenced by a wide variety of physical processes, most of which depend in some way on local environment. Since even within a single cluster, local mass densities, galaxy interaction rates, and galaxy velocity with respect to the hot inter galactic medium vary significantly as a function of location, correlations of galaxy properties with specific environmental features can reveal what mechanisms are responsible for galaxy transformation (e.g., Boselli \& Gavazzi 


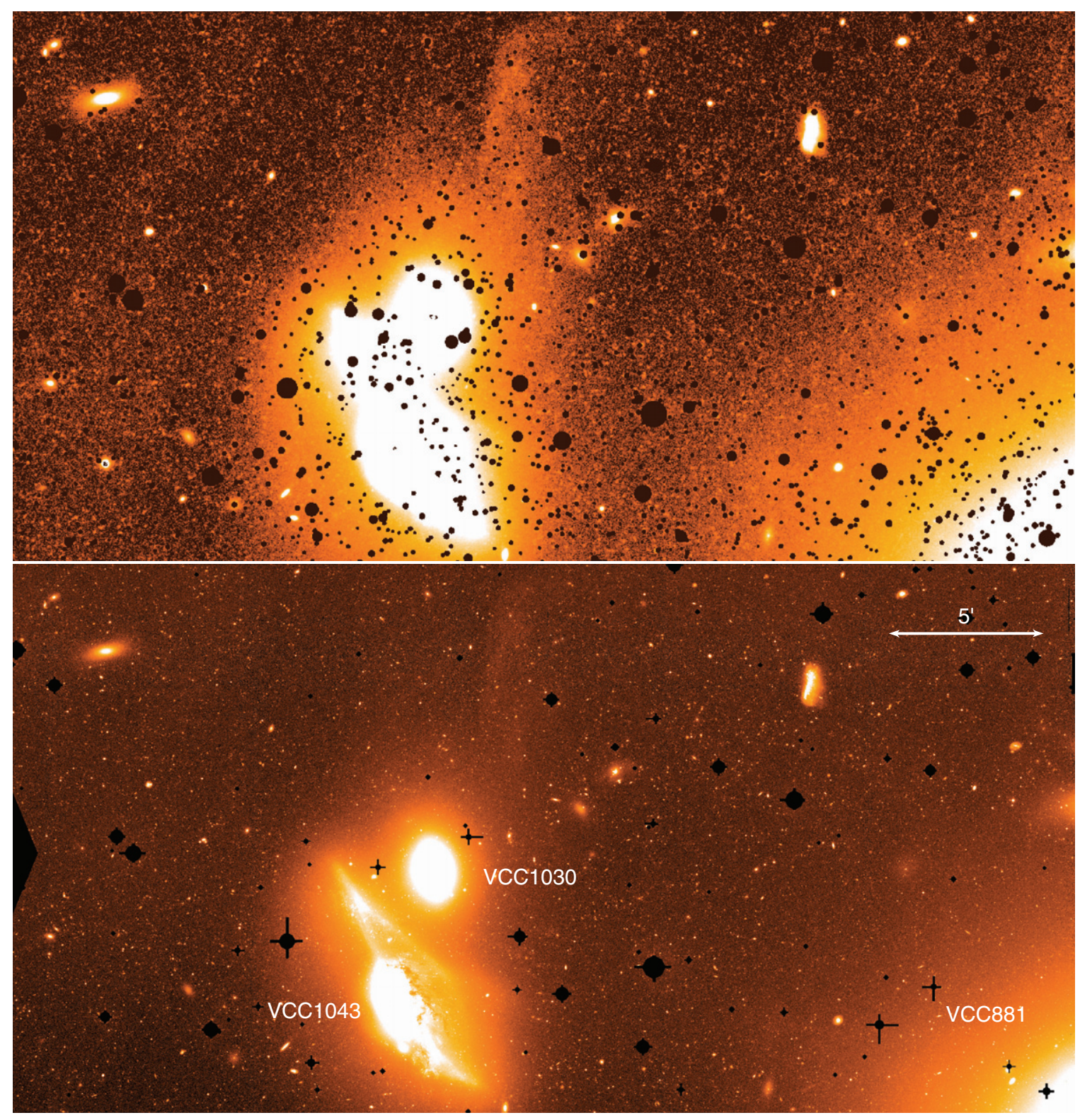

Figure 23. Diffuse light in the Virgo cluster. The two panels show a 17' $\times 34^{\prime}$ region near the cluster center that includes VCC1030, VCC1043, and VCC881 (labeled in the lower panel). The upper panel shows the $V$-band image from the deep imaging of Mihos et al. (2005), which was taken using a single SITe $2048 \times 4096 \mathrm{CCD}$, with a pixel scale of 1". 4 , on the $0.6 \mathrm{~m}$ Burrell Schmidt telescope, an instrument that is optimized for studying extended, low surface brightness features. The lower image is a $g$-band stack from the NGVS after combining the dithered and stepped exposures with Elixir-LSB (C. Cuillandre et al. 2012, in preparation). Bright stars have been masked in the NGVS image using a combination of the automask task in the THELI data reduction package (Erben et al. 2005, 2009) and customized software that masks CCD bleed trails (see Section 4.3). Most of the low surface brightness features visible in the upper image are also apparent in NGVS, which is shown here at full resolution (unbinned), with 0 .' 187 pixels.

2006). For instance, the spatial distributions of faint redsequence and star-forming galaxies provide information about how and where the quenching of star formation occurs (see Section 5.1.2). Correlations between position in the CMD and radial distance from the cluster center may indicate a sensitivity to the global cluster potential (e.g., Balogh et al. 2000), while correlations with intracluster medium density may be evidence for ram-pressure stripping (e.g., Vollmer et al. 2001; Poggianti et al. 2004; Moran et al. 2007).

Virgo is an excellent laboratory for studying in detail the role played by environment in galaxy evolution. Galaxy surface densities vary dramatically within the cluster, falling by nearly two orders of magnitude from their peak values from the (sub)cluster centers to the cluster boundary (see, e.g., Schindler et al. 1999); the electron density profile derived from X-ray observations of Virgo's hot intracluster gas shows a similar decrease (Nulsen \& Böhringer 1995). The NGVS sample of several thousand (mostly low- and intermediate-mass) galaxies, distributed over the full range in cluster radius, allows a thorough investigation of how galaxy evolution is affected by environment. The key elements of the NGVS in this context include: (1) depth, which allows galaxies with stellar masses as low as a few million solar masses to be detected and characterized (since these galaxies are very fragile with respect to external perturbations, they are ideal targets to study environmental effects); (2) spectral energy distribution (SED) coverage, which provides broad constraints on the past history of star formation and chemical enrichment; and (3) spatial resolution, which allows the structure and 

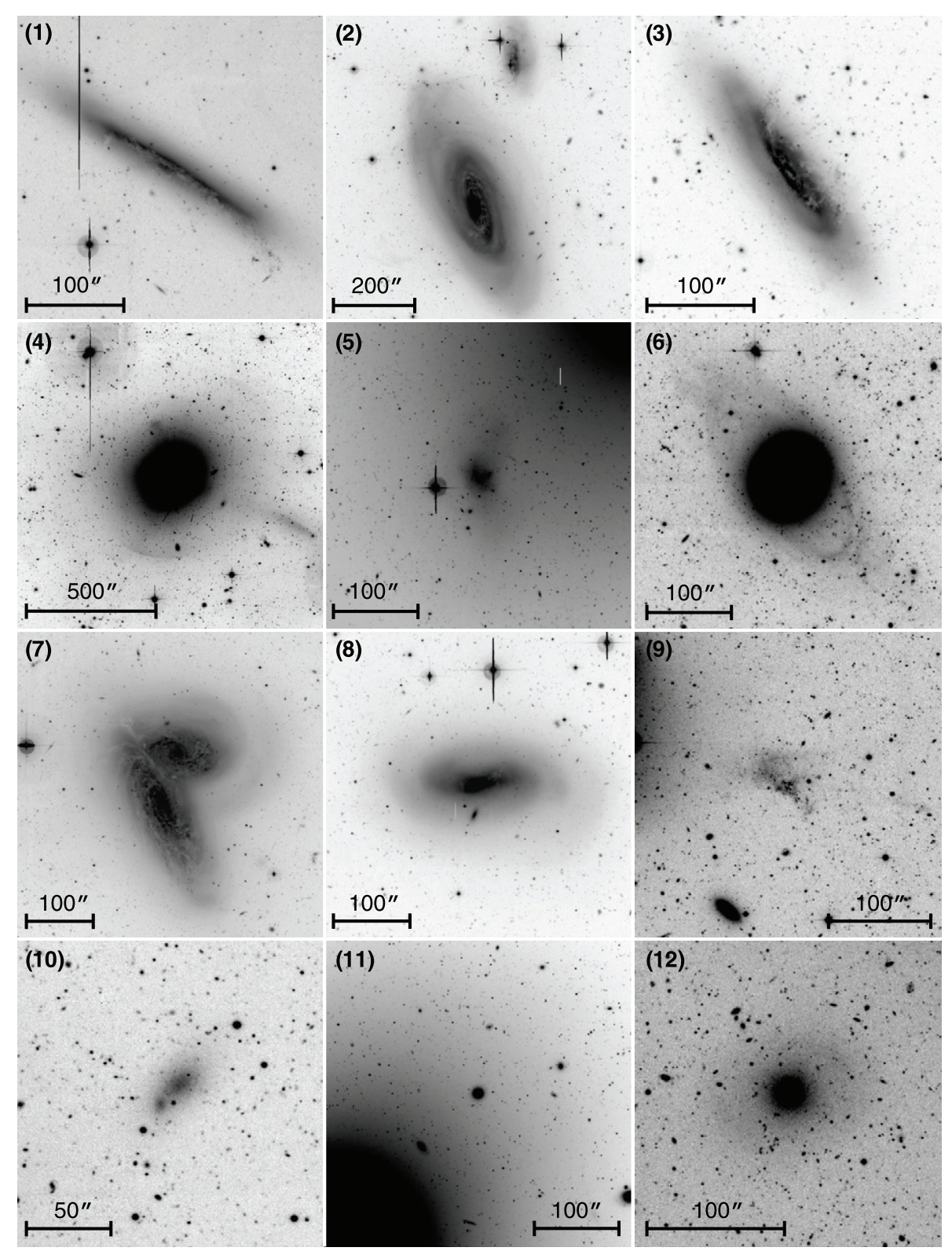

Figure 24. Virgo cluster as a laboratory for studying the effects of interactions and environment on galaxy evolution. The different panels show likely examples of various evolutionary processes at work. Panels (a)-(c): a ram-pressure-stripping sequence of VCC630, VCC1690, and VCC1516, illustrating gas stripping before, during and after its peak intensity (as inferred from H I observations). Panel (d): VCC1632, a remnant of a gas-poor merger. Panel (e): VCC1249, a gas-rich dwarf being accreted by M49 and an example of a "wet" accretion event. Panel (f): VCC355, an S0 galaxy with an extended star-forming ring, perhaps triggered by tidal interactions. Panel (g): VCC1673 and VCC1676, an interacting pair with tidally triggered star formation. Panel (h): VCC979, a possible post major merger Sa galaxy. Panel (i): VCC2062, a candidate tidal dwarf system. Panel (j): VCC1786, a possible binary dwarf system. Panel (k): VCC1199, a close companion of M49 that has likely undergone severe tidal stripping. Panel (1): VCC1681, a faint dwarf elliptical galaxy that shows faint spiral arms, possible evidence for the transformation by the tidal forces acting in the cluster environment. All images are in the $g$ band; the scale is as shown in each panel ( $100^{\prime \prime}$ corresponds to $\sim 8 \mathrm{kpc}$ at the distance of the Virgo cluster).

morphology of individual galaxies to be examined down to scales of $\sim 50$ pc or less.

Figure 24 shows a selection of galaxies from the NGVS chosen to illustrate some of the familiar processes that drive galaxy evolution. The panels are as follows.

1-3. A sequence of three galaxies-VCC630, VCC1690 (M90), and VCC1516-which are being actively ram-pressurestripped, as indicated by H I data; these objects illustrate the process of gas stripping before, during and after peak intensity (see, e.g., Boselli et al. 2006; Vollmer 2009, and references therein).
4. VCC1632 (M89), a massive early-type galaxy with a complex network of shells and plumes that are thought to have formed in a series of gas-poor mergers (Janowiecki et al. 2010).

5. VCC1249, a gas-rich dwarf that is being accreted by M49 in an example of "wet" accretion, i.e., one involving gas (Lee et al. 2000).

6. VCC 355, an early-type galaxy with an extended starforming ring, perhaps triggered by tidal interactions (Bettoni et al. 2010).

7. VCC1673 and VCC1676, a pair of interacting galaxies with tidally triggered star formation (Koopmann \& Kenney 2004). 
8. VCC979, a late-type system showing some evidence for a recent major merger (Kenney et al. 1996; Cortés et al. 2006).

9. VCC2062, a candidate tidal dwarf system associated with an early-type giant (Duc et al. 2007).

10. VCC1786, a possible binary dwarf system.

11. VCC1199, a close companion of M49 that has likely undergone significant tidal stripping (McLaughlin et al. 2012).

12. VCC1681, an early-type dwarf galaxy with faint spiral arms, evidence for transformation by the tidal forces acting in the cluster environment.

While this list is far from exhaustive, it is representative of the diversity of physical processes in Virgo that can be identified using deep, homogeneous, multi-color imaging. An important component of our study of galaxy evolution in the NGVS (i.e., Sections 5.1.2, 5.1.3, 5.1.5, and 5.1.6) will therefore involve the careful examination of morphology and structure (through 2D modeling, unsharp masking, and adaptive histogram equalization, etc.), which will be used to assess the importance of interactions or environmental processes in shaping individual galaxies.

\subsection{Foreground Science}

\subsubsection{A Deep Survey of the Kuiper Belt and Outer Solar System}

Being centered at relatively low ecliptic latitudes $\left(\beta \approx 14^{\circ}\right.$, with coverage from $7^{\circ}$ to $20^{\circ}$ ), NGVS images are well suited for the study of outer solar system objects. Although there is no pre-defined cadence built into the acquisition of NGVS data, the step-dither procedure adopted to characterize the scattered light contamination (Section 3.5) implies that in each filter, images of several fields are acquired during a single night, and that the same fields are then imaged again multiple times over the course of several days or weeks. This observing strategy gives a temporal sampling that is, somewhat fortuitously, highly effective for the detection and tracking of outer solar system objects (see Figure 25). Acquired over timescales of days, months, and years, the NGVS images provide tracking observations that are unbiased by ephemeris predictions, an important point for characterized Kuiper Belt surveys (see Kavelaars et al. 2009). The large contiguous area of the survey protects against the loss of objects as they move between fields, since only a small fraction of sources move outside the full survey region. To guard against an "orbit sample bias" induced by object loss at the survey boundaries, we have undertaken follow-up programs using both MegaPrime on CFHT and the MegaCam instrument on the Magellan $6.5 \mathrm{~m}$ Clay telescope. Each of these cameras offer wide-field coverage $\left(1^{\circ} \times 1^{\circ}\right.$ and $0.4 \times 0.4$, respectively), reducing the dependence of successful recovery on ephemeris predictions and protecting against tracking biases in our final orbit catalog. The ensemble of NGVS and related programs aimed at the study of the outer solar system is collectively known as the Deep Outer Solar System Survey (DOSSS). During the first three years of observations, DOSSS has detected and tracked 75 objects from a variety of subpopulations within the Kuiper Belt, including the second most distant known object (after Sedna; Brown et al. 2004) on an orbit that is not subject to scattering encounters with Neptune.

DOSSS is providing a new and significant structural probe of the Kuiper Belt. Previous Kuiper Belt Object (KBO) surveys have covered nearly the entire northern sky to limits of $g \simeq$ 22 mag (Schwamb et al. 2010), while ecliptic surveys have

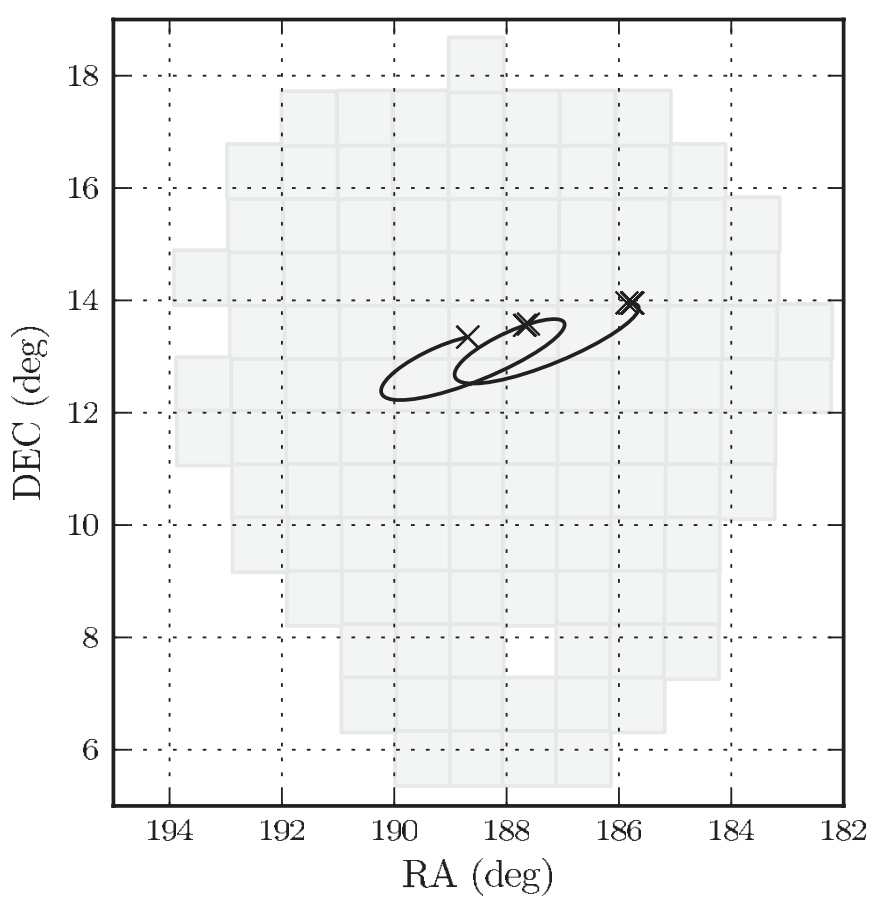

Figure 25. Sky trajectory of a representative trans-Neptunian object (TNO) discovered in the NGVS. The crosses mark the locations where the TNO was detected, with the line showing the complete motion of the object over the 2009-2011 observing seasons (moving from west to east). Since the NGVS imaging (shaded area) is being acquired over multiple years, it enables the detection of the moving source in multiple observing seasons. The orbital parameter uncertainty on this source is less than $1 \%$, which is adequate for orbit classification.

probed hundreds of square degrees to depths of $g \simeq 24 \mathrm{mag}$ (Elliot et al. 2005; Kavelaars et al. 2009). In comparison, individual NGVS exposures reach a depth of $g \simeq 25.9$ mag over a $104 \mathrm{deg}^{2}$ region, allowing one to probe a much fainter KBO population. Moreover, the NGVS fields, which are centered at a heliocentric longitude and latitude of $\sim 175^{\circ}$ and $\sim+14^{\circ}$, respectively, are well placed to examine the structure of the $\mathrm{n}: 2$ resonant populations. ${ }^{46}$ Based on 24 and five detections, respectively, the Canada France Ecliptic Plane Survey (CFEPS) de-biased model (i.e., accounting for the relative probability of detections) of the 3:2 and 5:2 resonant populations found that the sizes of these two resonances are nearly equal, a somewhat surprising result that was not predicted by current models for the evolution of the outer solar system (Gladman et al. 2012). Based on the CFEPS resonant model, DOSSS is expected to detect approximately 20 3:2 members and, because of its greater depth, a nearly equal number of 5:2 resonant objects, providing a robust measurement of the ratios of these resonant populations.

DOSSS is also contributing to the study of the size distribution of the members of the Kuiper Belt, a still highly debated subject. The NGVS single exposure point-source depth reaches down to sizes of $H_{g} \simeq 11.5 \mathrm{mag}^{47}$ for KBOs at distances of $30 \mathrm{AU}$ (a typical value for the pericenter distance of resonant KBOs). This is about 2 mag fainter than previous surveys, probing object sizes below the putative break in KBOs size distribution that is thought to occur near $H_{g} \sim 10$ mag (Fraser \& Kavelaars 2008; Fuentes $\&$ Holman 2008). Because the NGVS fields are located several

\footnotetext{
46 See Gladman et al. (2012) for a discussion of the coupling between sky coverage and survey direction.

47 In planetary science, sizes are generally referred to in terms of "absolute H magnitudes," defined as the magnitude the object would have if placed at $1 \mathrm{AU}$ from the Sun and 1 AU from Earth.
} 
degrees north of the ecliptic plane, the observations provide a direct probe of the "hot" component of the Kuiper Belt, free from contamination by the "cold" classical objects. As the size distribution functions for the hot and cold populations appear to differ, isolating a sample that probes just one component will provide important new information. Given the high-quality orbital and distance information that DOSSS will provide, we will be able to compare directly the size distribution functions of the resonant and non-resonant Kuiper Belt populations.

\subsubsection{Structure of the Galactic Halo}

In $\Lambda$ CDM models of structure formation, the DM halo of the Milky Way galaxy is assembled hierarchically through the accretion and merging of subhalos (e.g., Klypin et al. 1999; Moore et al. 1999). Only a fraction of these subhalos are thought to have accreted gas and formed stars, and thus be visible today as dwarf galaxies (see, e.g., Benson et al. 2003). According to numerical simulations, a subset of these dwarfs will subsequently be disrupted by Galactic tides, spilling their stars into the Milky Way and forming at least part of its stellar halo (e.g., Bullock \& Johnston 2005; Abadi et al. 2006; Cooper et al. 2010). Depending upon the details of the individual accretion events, the fossil records of this halobuilding process - such as stellar streams or the surviving cores of satellites-may still be visible today. The outer halo of the Galaxy represents a unique laboratory, both because of its proximity and because the phase mixing timescales can exceed the Hubble time; indeed recent wide-field surveys of the Milky Way (and M31; McConnachie et al. 2009), particularly the SDSS (e.g., Belokurov et al. 2006), have qualitatively confirmed this basic picture. Recently discovered stellar substructures, or "overdensities," in the Galactic halo include $~ 15$ low surface brightness dwarf galaxies, as well as several new GCs and stellar streams.

Of the known large-scale substructures in the Galactic halo, there are two that are of particular relevance to the NGVS. The first is the Sagittarius Stream, a tidal feature that extends across the entire sky (Ibata et al. 2001). Belokurov et al. (2006) showed that the stream appears to bifurcate into two distinct branches at $\alpha_{2000} \sim 190^{\circ}$ and $\delta_{2000} \sim+10^{\circ}$ (i.e., formally consistent with lying inside the NGVS survey region). The physical explanation for this bifurcation remains unclear: It could be the sign of distinct wrappings of the stream from earlier orbits (e.g., Fellhauer et al. 2006) or, in part, be due to a misalignment of the rotational and orbital angular momenta in a progenitor system that had at least some intrinsic rotation (Peñarrubia et al. 2010, 2011).

The second substructure is the Virgo Overdensity (VOD), which was first noted by Jurić et al. (2008) using a photometric SDSS parallax technique to construct a 3D tomographic map of the Galaxy. These authors identified a coherent overdensity, relative to a smooth Galaxy model, toward $(l, b) \sim\left(300^{\circ}, 65^{\circ}\right)$, or $\sim 11^{\circ}$ from M87. This feature, which may cover an area of up to $\sim 1000 \mathrm{deg}^{2}$ in the general direction of Virgo, stands out as a factor of $\sim 2$ enhancement relative to the mean density at Galactocentric radii of $6-20 \mathrm{kpc}^{48}$ Subsequent studies of the VOD have led to differing interpretations. Martínez-Delgado et al. (2007) have suggested that it is a new detection of the Sagittarius Stream, as predicted by some dynamical models.

\footnotetext{
48 Note that this feature should not be confused with the independently discovered Virgo Stellar Stream (Duffau et al. 2006); it is not clear if the two features are related (e.g., Vivas et al. 2008).
}

However, this interpretation is disputed by Newberg et al. (2007), who traced Sagittarius debris using turnoff F stars, and Vivas et al. (2008), who argued that the VOD is in fact made up of several distinct substructures.

The placement of the NGVS is well suited to investigations of both the VOD and the Sagittarius Stream at its bifurcation point. The survey's depth (about 2-3 mag below that of SDSS) and excellent imaging quality (which facilitates star-galaxy separation at faint magnitudes) makes it possible to identify with relative ease the various stellar components in the CMD (see Figure 26). In addition to providing important new information on the surface brightness, luminosity, and spatial structure of the VOD and Sagittarius Stream, star counts based on traditional halo tracers such as blue horizontal branch stars and RR Lyraes should provide important new constraints on the mean density profile of the halo. Understanding the nature of the Sagittarius Stream provided the motivation for a Very Large Telescope (VLT) FLAMES follow-up program, briefly described in Section 6.

Finally, as noted above, the SDSS has proved to be a remarkably powerful tool for the detection of halo overdensities (i.e., faint dwarf galaxies and distant star clusters) thanks to its enormous areal coverage of $\approx 10,000 \mathrm{deg}^{2}$. Although this exceeds that of the NGVS by two orders of magnitude, this difference is partly compensated by the greater depth of the NGVS. For instance, the CMDs of resolved overdensities in the Galactic halo can be traced in the SDSS and NGVS to $g \sim 23 \mathrm{mag}$ and $\sim 25.9 \mathrm{mag}$, respectively. For old, metalpoor populations, the main-sequence turnoff has an absolute magnitude of $M_{g} \simeq 4.2$ (An et al. 2009), meaning that such stars could be detected in the NGVS out to distances of $\sim 182 \mathrm{kpc}$ (compared to $\sim 58 \mathrm{kpc}$ in the SDSS). Thus, the volume of the Milky Way halo probed by the NGVS is $\sim 1 / 7$ th that of the SDSS, so that a comprehensive search for low surface brightness dwarfs and faint GCs within the NGVS could yield interesting candidates. ${ }^{49}$

\subsection{Background Science}

\subsubsection{Weak Gravitational Lensing}

From the mid-1980s, galaxy-galaxy lensing (i.e., the weaklensing signal in a background galaxy due to a lens that is itself a galaxy) and, later, cosmic shear (the observed alignment of background galaxies produced by large-scale structure in the universe) have emerged as powerful tools with which to probe the masses and shapes of DM halos and constrain cosmological parameters. The potential of galaxy-galaxy lensing was first demonstrated by Brainerd et al. (1996) and Hudson et al. (1998), who were able to measure extremely weak shear by stacking the lensing signal from individual foreground galaxies. More recent studies have been able to exploit galaxy-galaxy lensing to study the connection between the DM halo and the stellar population of the lens by selecting lenses according to their color and morphological type (Mandelbaum et al. 2006a; van Uitert et al. 2011); the further addition of radio or X-ray observations enables AGNs to be brought into the analysis (e.g., Mandelbaum et al. 2009). On larger scales, weak gravitational lensing has been used to study halos at group and cluster scales (Hoekstra

\footnotetext{
49 At least 10 halo "overdensities" are found within 20 deg of the NGVS field center: Bootes I, Bootes II, Canes Venatici I, Canes Venatici II, Pal 4, Coma Berenices, Leo II, Leo IV, Leo V, and Koposov. Note that this list does not include extended features, such as the VOD and Sagittarius Stream discussed above.
} 


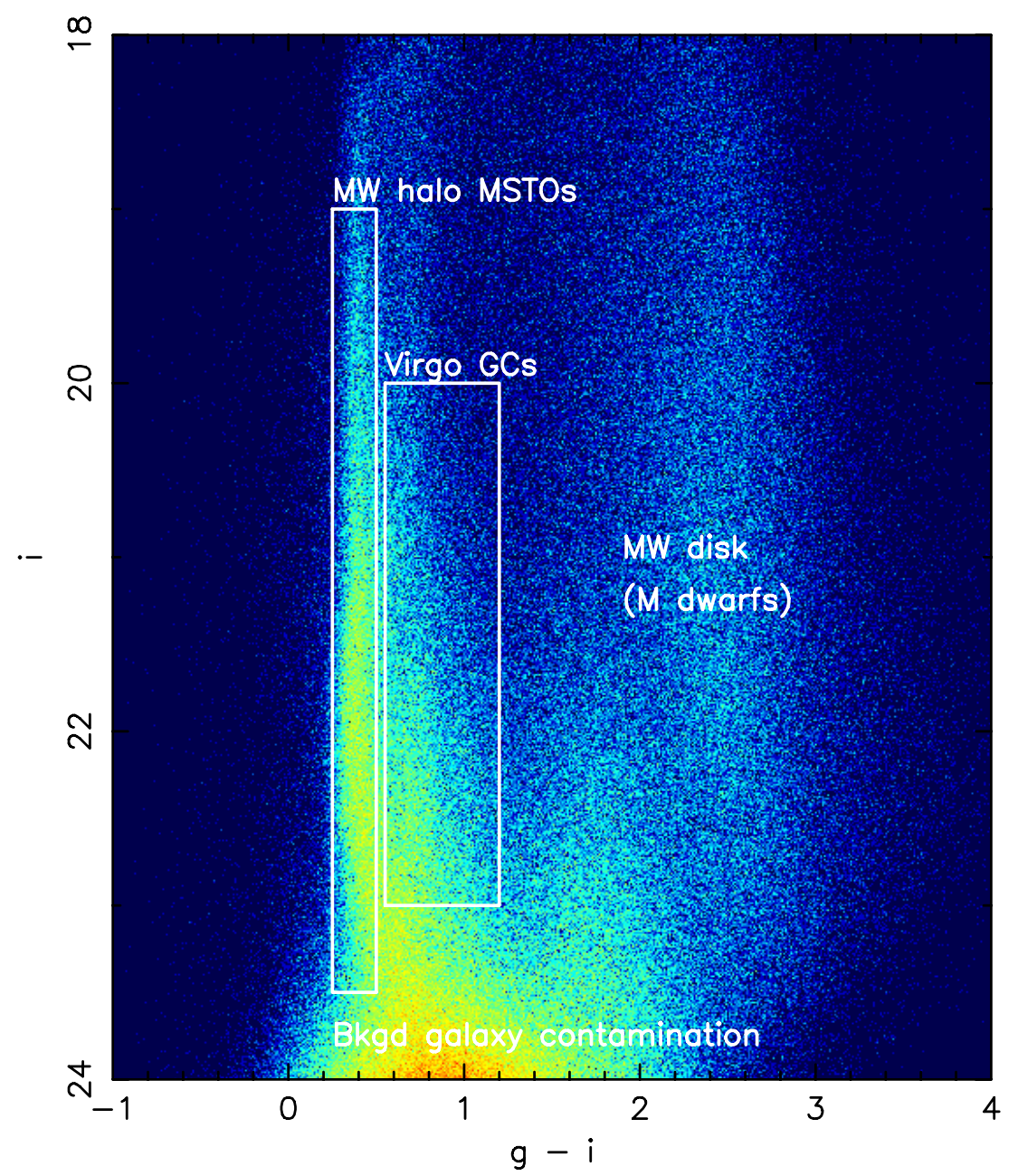

Figure 26. Hess diagram, showing the relative density of occurrence of stars in the $i$ magnitude vs. $g-i$ color plane, based on $g i$ imaging for 14 million compact sources detected in the NGVS. Several prominent features are labeled, including the locus defined by the main-sequence turnoff stars in the Galactic halo, faint (mostly M dwarf) stars belonging to the Galactic disk, GCs in the Virgo cluster, and a large population of faint, compact background galaxies. The NGVS line of sight passes through a slice of the Galactic halo that includes the Sagittarius stream and the Virgo Overdensity. At the limiting magnitude of the NGVS, metal-poor main-sequence turnoff stars in the halo are visible to a distance of $\sim 180 \mathrm{kpc}$.

et al. 2001; Parker et al. 2005; Johnston et al. 2007), with the most recent studies showing how groups and cluster lensing can be used to constrain halo and galaxy formation models (Leauthaud et al. 2010, 2011).

The viability of performing weak-lensing measurements using MegaPrime data has been amply demonstrated by the CFHT Legacy Survey (CFHTLS; Hoekstra et al. 2006; Benjamin et al. 2007; Parker et al. 2007; Fu et al. 2008). The NGVS reaches similar depths and has observing constraints similar to the CFHTLS, and thus is well suited for weak-lensing studies. Additionally, it has certain characteristics that other optical surveys of similar depth do not have. First, it offers the largest contiguous field ever observed at these depths (although the CFHTLS covered a total area of $170 \mathrm{deg}^{2}$, this was divided among four separate fields, with the widest field being $64 \mathrm{deg}^{2}$ ): compared with other cosmic shear surveys, the NGVS will therefore provide better constraints on the matter power spectrum on wide scales $\left(\sim 8^{\circ}\right)$.

Second, the NGVS region is very rich in foreground galaxies, and it therefore provides an excellent test case for the measurement of the lensing signal in the presence of significant foreground contamination. Analyzing the distortion (shear) signal under these conditions has not yet been attempted: Previous surveys have preferred empty, blank fields since foreground objects generate large-scale, low-amplitude light gradients, which could contaminate the cosmic shear signal. Weak-lensing analysis of the NGVS data will therefore be particularly relevant for future lensing analyses in all-sky surveys where high foreground contamination cannot be avoided (e.g., LSST and Euclid).

Third, the wealth of complementary data at other wavelengths (Section 2.2) will give the NGVS a clear advantage for galaxy-galaxy lensing studies, by allowing the foreground lenses to be selected based on specific properties (e.g., the amount of star formation, the dust content, and the presence of AGN activity), and therefore allowing a study of the connection between host galaxies and DM halos as a function of galaxy properties. The NGVS will be able to address the question of halo flattening, which provides a direct test of the nature of DM (Dubinski \& Carlberg 1991). Although some attempts were made at measuring the halo flattening in recent surveys (Mandelbaum et al. 2006b; Hoekstra et al. 2004; Parker et al. 2007), the results obtained so far remain contradictory.

Fourth, the NGVS will allow us to employ not only the shear introduced by gravitational lensing in the images of background galaxies, but also the magnification. It has been shown recently (Scranton et al. 2005; Hildebrandt et al. 2009, 
2011; Ménard et al. 2010; van Waerbeke 2010; van Waerbeke et al. 2010) that it is possible to use the additional information in the magnification to arrive at more precise measurements than with shear alone, to perform cross-checks for systematic errors, and to conduct some unique measurements that would not otherwise be possible. The NGVS with its extensive, accurately calibrated multi-band data represents an ideal survey to measure magnification, which requires exquisite photometry. As in the shear case, it will be important to measure the magnification in a non-empty field for the first time, where, e.g., light halos of foreground objects cannot be neglected. A special class of magnification measurements employing the magnitude-shift effect (Ménard et al. 2010) will allow us to study the dust halos around foreground galaxies. The NGVS gives us the opportunity to study this with many well-resolved, low-redshift, low-mass lenses where the magnification will be subdominant to the dustdimming, making it easier to measure the latter.

Finally, the NGVS data will allow an investigation of the intrinsic alignment of galaxies and its effect on cosmic shear measurements. It is well known that galaxies tend to be aligned with the local gravitational field, as an end process of the complicated interplay between galaxy formation, halo tidal force, and merging. Indeed, in the Virgo cluster, West \& Blakeslee (2000) found marginal evidence that bright ellipticals are aligned with the clusters' main axis. It is also well known that intrinsic alignment contaminates the cosmic shear signal, although it has a distinct signature in redshift and angular dependence compared with cosmic shear. This signature can be used to distinguish between intrinsic alignment and cosmic shear (Joachimi \& Schneider 2008, 2009), and recent measurements show that this is indeed possible (Joachimi et al. 2011; Mandelbaum et al. 2011). The NGVS offers a unique laboratory for measuring the intrinsic alignment in a cluster environment, and such measurements are relatively straightforward given the relative proximity, and therefore good resolution, of galaxies in the Virgo cluster.

\subsubsection{Background Galaxy Clusters}

As the largest collapsed structures in the universe, galaxy clusters provide important constraints on both cosmological models and the evolution of galaxies located within the "hubs" of the cosmic web, where structures are assembled rapidly through accretion along filaments. Many of the high-redshift galaxy clusters known today were first detected in the X-rays with ROSAT, and then followed up with extensive optical and $\mathrm{X}$-ray observations in order to explore their galaxy populations, gas properties, and redshift distribution. However, the X-ray signal fades rapidly with redshift, so ROSAT was able to detect thousands of clusters at redshift $z \lesssim 0.5$, but only hundreds in the range $0.5 \lesssim z \lesssim 1$ and tens beyond $z \sim 1$. This situation is rapidly changing with the development of dedicated millimetre Sunyaev-Zel'dovich surveys covering large areas of the sky (Williamson et al. 2011).

With the development of mosaic cameras on 4-8 m class telescopes, large galaxy cluster surveys based on optical/IR observations have also begun to appear. More than 10,000 SDSS clusters have been detected up to $z \sim 0.3$ (Koester et al. 2007; Wen et al. 2009) while the red-sequence cluster survey of Gladders \& Yee $(2005)$ detected $\approx 1000$ clusters at $0.35<z<0.95$ over an area of $\approx 70 \mathrm{deg}^{2}$. Another thousand candidates have been detected in a similar redshift range using data from the CFHTLS (Adami et al. 2010; Milkeraitis et al. 2010) while a few tens of candidates at $z \gtrsim 1$ have also detected by combining these surveys with imaging from Spitzer (e.g., Wilson et al. 2008, 2009b; Muzzin et al. 2009).

With the depth and SED coverage of the NGVS, we expect 5-10 clusters deg $^{-2}$ with masses above $\mathcal{M} \sim 5 \times 10^{13} \mathcal{M}_{\odot}$ over the range $0.1<z<1$, giving a total of 500-1000 clusters. Figure 27 shows examples of clusters in the range $0.4 \lesssim z \lesssim 0.8$ identified in the NGVS using the 3D-Matched-Filter cluster finding algorithm of Milkeraitis et al. (2010) and an independent red-sequence-based algorithm (Mei et al. 2012). Based on a preliminary analysis, and on results from surveys with similar depth and SED coverage (e.g., Coupon et al. 2009), we conclude that it will be possible to measure reliable photometric redshifts and select cluster populations to a depth of $i \approx 22.5 \mathrm{mag}$. This large cluster sample will be a powerful database with which to address some key questions in galaxy evolution, two of which we discuss here.

First, the massive early-type galaxies at the centers of many clusters are thought to grow by repeated mergers and accretions as dynamical friction drives galaxies toward the cluster center. Recent work on the SDSS galaxy sample (Bernardi et al. 2011a, 2011b) has shown that the merger history of these galaxies can be derived from their measured sizes, masses, and velocity dispersions. The NGVS sample-combined with follow-up high-resolution imaging and optical/IR spectroscopy-will allow an examination of the assembly of early-type galaxies and a comparison to cosmological models. Second, at lower masses, quenching and morphological transformation of infalling, starforming galaxies is expected to play a role in the build up of the red sequence; work at $z<0.4$ (e.g., Lu et al. 2009; HuertasCompany et al. 2009) suggests that most of the faintest redsequence galaxies are accreted onto the clusters at $z<0.2$, while the faint end of the luminosity function shows relatively little evolution between $z \sim 0.2$ and $z \sim 0.4$ (see also Harsono $\&$ De Propris 2009). However, there remains some disagreement over the efficiency of the quenching and morphological transformation mechanisms at higher redshift. Some studies point to a paucity of faint red galaxies, suggesting that these fainter objects were accreted at $0.5<z<1$ (De Lucia et al. 2007), whereas other work shows little evolution in the low-mass end of the red sequence (Andreon 2008). The NGVS sample should contain many clusters in this redshift range, allowing the lowmass end of the red sequence to be examined uniformly from $z \sim 1$ to the present.

\subsubsection{Strong-lensing Events}

Strong lenses represent the most extreme manifestation of the gravitational bending of background (source) light by intervening structures (lenses). Such events-which are characterized by multiple or strongly distorted images arising from the different paths taken by the source photons traveling to the observer-are powerful tools for probing the distribution of DM and baryons within DM halos that range in scale from individual galaxies to rich clusters. With the deepest and widest contiguous coverage of any optical survey yet undertaken, the NGVS provides an excellent opportunity to assemble a large, homogeneously defined sample of strong gravitational lenses, effectively extending the work done with CFHTLS data (Cabanac et al. 2007; More et al. 2012).

A systematic search for galaxy-scale strong-lensing events in the NGVS data is being carried out as described by Cabanac et al. (2007) and implemented in R. Gavazzi et al. (2012, in preparation). Several automated procedures can be used to search efficiently for strong-lensing systems-either in the 


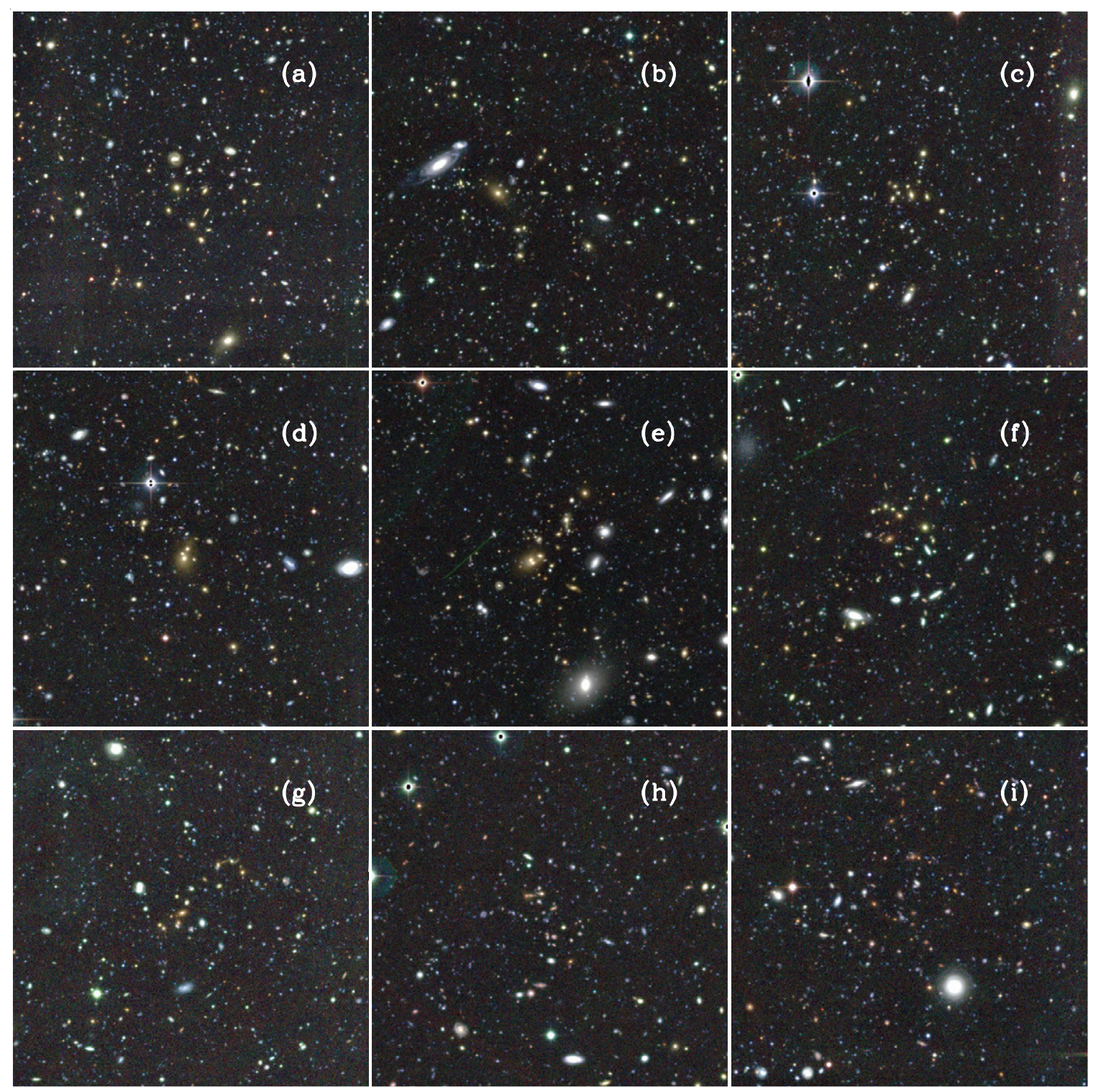

Figure 27. Panels (a)-(i): color images of nine background clusters identified in the NGVS using the 3D-Matched-Filter cluster finding algorithm of Milkeraitis et al. (2010) and the red-sequence-based algorithm of Mei et al. (2012). Cluster photometric redshifts increase from $z \sim 0.4$ in panel (a) to $z \sim 0.8$ in panel (i). Each image measures $1.5 \times 1.5 \mathrm{Mpc}$ in size. The NGVS should yield 500-1000 clusters with $\mathcal{M} \gtrsim 5 \times 10^{13} \mathcal{M}_{\odot}$ in the range $0.1 \lesssim z \lesssim 1$.

form of rings, arcs, or multiplets-while photometric redshift codes can be used to provide provisional estimates for the redshift of the putative lens. In the case where the deflecting galaxies correspond to bright, early-type galaxies in the redshift range $0.1 \lesssim z \lesssim 0.9$, we expect $\sim 3000$ such systems per square degree; extrapolating from the CFHTLS, a total of $\sim 200$ candidate gravitational lenses should be detectable over the full NGVS survey area, with splitting angles in the range $\sim 2^{\prime \prime}-15^{\prime \prime}$ (see Figure 28). Ultimately, these candidates will require highresolution HST imaging for confirmation and/or spectroscopy to measure unambiguously the redshift of the foreground lensing galaxy and the faint background source imaged into a complete or partial Einstein ring.

The expected population of $\sim 200$ lenses from the NGVS, when combined with the 330 candidates present in the CFHTLS (R. Gavazzi et al. 2012, in preparation) in the same $0.1 \lesssim z \lesssim$ 0.9 redshift range, will supplement the low-redshift sample of galaxy-scale strong lenses from the SLACS project (Auger et al. 2010). Once combined with stellar dynamical measurements for the lens galaxies (Ruff et al. 2011), this database will allow a detailed investigation into the dynamical processes at work in the secular evolution of massive early-type galaxies in a wide range of environments.

\section{SUPPLEMENTARY PROGRAMS}

To better leverage the ability of the NGVS data to address the topics summarized in Section 5, a number of spectroscopic and imaging programs have been undertaken and are at various stages of completion/execution. These programs are briefly outlined below; full details will be provided in future publications.

I. Optical spectroscopy. Several spectroscopic programs, currently underway, capitalize on the census of Virgo GCs, compact objects, and Galactic halo stars enabled by the NGVS (see Sections 5.1.4, 5.1.6, and 5.2.2).

The study of galaxy kinematics, DM distribution, and orbital anisotropy of baryonic substructures motivated three complementary projects at the MMT, VLT, and Magellan. Baryonic tracers in the M87 and M49 regions were targeted during a 


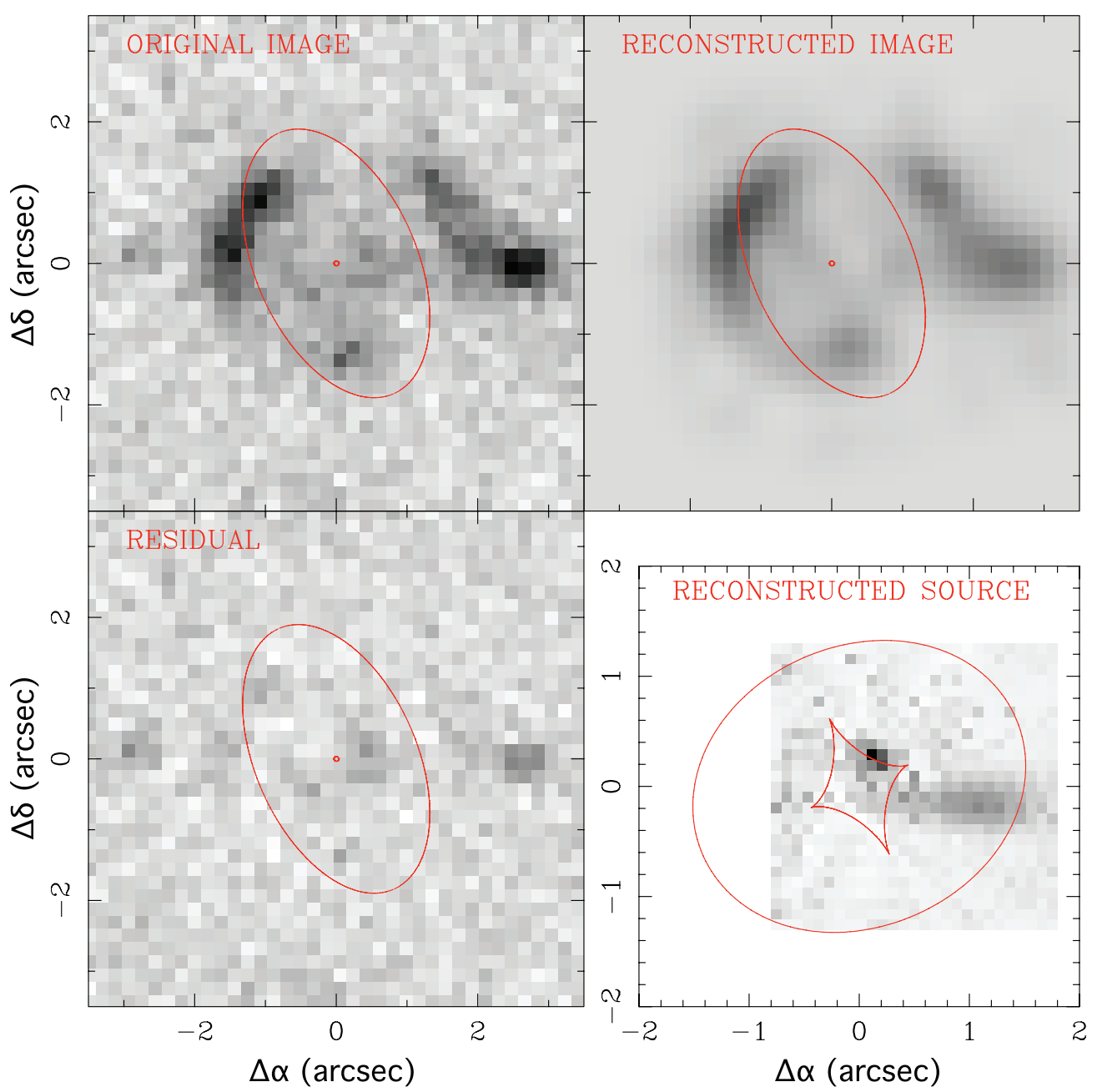

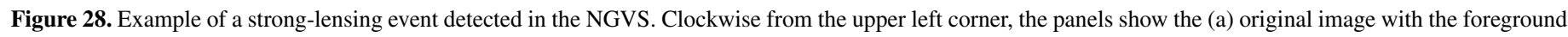

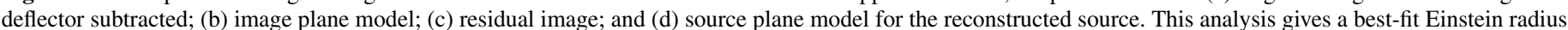

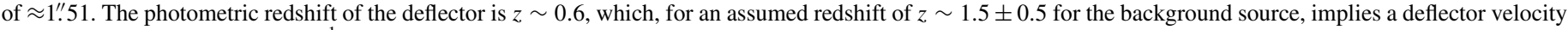
dispersion of $\sigma \sim 320 \pm 30 \mathrm{~km} \mathrm{~s}^{-1}$. The NGVS is expected to uncover 200 such strong-lensing events.

(A color version of this figure is available in the online journal.)

6.5 night observing campaign in 2009/2010 using Hectospec at the MMT (spectral range $\lambda=0.36-0.92 \mu \mathrm{m}$, spectral resolution $R \sim 1000$ ), and during two nights in 2012 February using IMACS at Magellan $(\lambda=0.42-0.56 \mu \mathrm{m}, R \sim 750)$. The Hectospec data produced radial velocities for a magnitude-limited ( $g \leqslant 19.5 \mathrm{mag}$, corresponding to $M_{g} \leqslant-11.6 \mathrm{mag}$ ) sample of $~ 900$ GCs and dwarf galaxies, some as far as $800 \mathrm{kpc}$ from the cluster center, ${ }^{50}$ while the Magellan program extended this sample to include $\sim 4000 \mathrm{GCs}$, cEs, and UCDs within $200 \mathrm{kpc}$ of M87 to a limiting magnitude of $g \leqslant 23.2 \mathrm{mag}$ (i.e., sampling the brightest third of all GCs in the Virgo core). An approved program at VLT VIMOS $(\lambda=0.48-1.0 \mu \mathrm{m}, R \sim 580)$ will expand these studies to the halos of 12 massive $\left(\log M / M_{\odot} \geqslant 10.8\right)$ early-type Virgo galaxies, measuring radial velocities for a total of $\sim 3000 \mathrm{GCs}$ as faint as $g=23.0 \mathrm{mag}$. The radial velocities derived from these programs, combined with X-ray mass profiles, will allow us to measure, simultaneously, the mass profile and the velocity ellipsoid of baryonic tracers in the critical transition region between M87 and cluster potential (e.g., Côté et al.

\footnotetext{
50 As a by-product, spectra for a randomly selected sample of $\sim 2100$ galaxies to a limiting magnitude $g=21.0 \mathrm{mag}$ (i.e., three magnitudes deeper than reached by the SDSS) are being used to calibrate the NGVS photometric redshifts and tighten the constraints on Virgo membership.
}

2001, 2003; Strader et al. 2011), and to trace the galaxy kinematics to galactocentric radii $R \gtrsim 5 R_{e}$ for the most massive cluster galaxies, thus building on previous studies that targeted the inner kinematic structure of early-type/red-sequence galaxies using IFU spectroscopy (e.g., ATLAS3D: Cappellari et al. 2011a, 2011b; Emsellem et al. 2011).

By targeting a magnitude-limited sample of GCs and compact sources selected from the NGVS images, the Magellan project mentioned above will also produce a systematic census of compact stellar systems (cEs and UCDs) in the cluster core. Two additional programs are specifically designed to explore the nature of compact stellar systems. An approved 2012 five night allocation at the Anglo-Australian Telescope will employ $2 \mathrm{dF}+\mathrm{AAOmega}(\lambda=0.41-0.61 \mu \mathrm{m}, R \sim 1480)$ to carry out a comprehensive spectroscopic survey of bright $(g \lesssim$ $21 \mathrm{mag}$ ) and compact (but resolved) sources over approximately half of the NGVS survey area (with plans to complete the coverage in 2013), thus providing the first ever complete census of compact, low-mass stellar systems in any environment and allow for a direct test of the predictions of tidal stripping versus hierarchical merging models for the origin of such system. In a complementary project, a detailed investigation of the internal kinematics and stellar populations of a complete sample of 19 
cEs will be conducted through a $\sim 50 \mathrm{hr}$ observing campaign with Gemini's GMOS IFU $(\lambda=0.41-0.55 \mu \mathrm{m}, R \sim 1688$, field of view $5^{\prime \prime} \times 7^{\prime \prime}$ ), scheduled for execution in the spring of 2012. The galaxies, selected from the NGVS images as $\geqslant 1 \sigma$ outliers in the magnitude-surface brightness relation shown Figure 16, have stellar masses in the range $(0.4-5.1) \times 10^{10}$ $M_{\odot}$; the program is therefore a natural extension to cEs of the ATLAS3D survey, and will yield 2D maps of rotation velocities, velocity dispersions, ages, and metallicities $([\mathrm{Z} / \mathrm{H}]$ and [alpha/Fe]), as well as dynamical masses, gradients in the stellar populations, angular momentum profiles, and a census of kinematic substructures, for these yet to be explored systems.

Lastly, the nature of the Sagittarius Stream (see Section 5.2.2) will be targeted in a dedicated VLT FLAMES ( $\lambda=0.50-0.58 \mu \mathrm{m}, R \sim 6500) 26 \mathrm{hr}$ campaign, scheduled for execution in 2012. The program will yield velocities for $\sim 600$ turnoff stars $(21.2 \mathrm{mag}<g<22.4 \mathrm{mag}$ ) color-selected from NGVS images as belonging to the stream, along two separate lines of sight through the Galactic halo.

II. Near-infrared Imaging. Expanding the SED coverage from the optical to the NIR is highly efficacious for stellar population studies (Puzia et al. 2002). To this aim, two large campaigns, collectively known as NGVS-IR, were initiated capitalizing on the large field of view, sensitivity, and image quality of the VISTA/VIRCAM (1.5 $\mathrm{deg}^{2}$ field of view, 0.34 pixels) and CFHT/WIRCam $(21.5 \times 21.5$ field of view, 0'.31 pixels) NIR imagers. The CFHT/WIRCam campaign, completed in 2010, provided deep $\left(\sim 23.0 \mathrm{AB}\right.$ mag) $K_{s}$ images of a $2^{\circ} \times 2^{\circ}$ region centered on M87. The VISTA/VIRCAM campaign, scheduled to be completed in the spring of 2012, will provide $J$ and $K$ images of a $3^{\circ} \times 2^{\circ}$ region centered on M49, at a depth comparable to what was reached in the M87 region $(\mathrm{T} . \mathrm{H}$. Puzia et al. 2012, in preparation). For both campaigns, the survey area was tiled with slightly overlapping pointings, each of which consists of a series of dithered exposures. The observing sequence is a variant of the NGVS step-dither strategy, where, at regular intervals during the dither pattern, the telescope is nodded to a separate field (still within the survey area) to guarantee good temporal sampling of the near-IR sky brightness variations and accurate modeling of steep surface brightness galaxy profiles.

The depth reached by NGVS-IR corresponds roughly to the turnover magnitude of the GC luminosity function in the critical central regions of Virgo's A and B subclusters. Combining the NGVS and NGVS-IR data yields optical-NIR colors with a photometric accuracy such that stellar population ages and metallicities in simple stellar systems (GCs, UCDs, early-type galaxies, and stellar nuclei) can be measured with a resolution of $\Delta t / t \sim 0.5$ and $\Delta Z \sim 0.3$ dex (Puzia et al. 2002). This allows us to investigate the ages and metallicity distribution functions of the GCs in the core of Virgo, to address whether the classic GC bimodality of optical colors is reflected in optical/NIR colors, and to examine the age-metallicity and mass-metallicity relations for UCDs. In addition, the $4000 \AA$ break in the spectra of red-sequence galaxies moves beyond the $z$ band at redshift $z \sim 1$, making such galaxies undetectable based on optical colors alone. The NGVS-IR, combined with the optical data, is proving effective in detecting galaxy clusters at larger redshifts (R. Munoz et al. 2012, in preparation).

\section{SUMMARY}

As the dominant mass concentration in the local universe and the largest collection of galaxies within $\sim 40 \mathrm{Mpc}$, the Virgo cluster plays a key role in understanding how galaxies form and evolve in dense environments. The NGVS capitalizes on the wide-field imaging capabilities of MegaPrime/MegaCam at the CFHT, combined with the excellent image quality of the site, to survey the Virgo cluster from its core to its virial radius (for a total coverage of $104 \mathrm{deg}^{2}$ ), in five filters ( $u^{*}$ griz), to a point-source depth of $g \sim 25.9 \mathrm{mag}(10 \sigma)$ and a corresponding surface brightness limit of $\mu_{g} \sim 29.0 \mathrm{mag} \operatorname{arcsec}^{-2}$. The NGVS will supersede all previous optical studies of this uniquely important system. Compared with the survey that currently serves as the standard reference for the Virgo cluster in the optical (the 1980's Virgo Cluster Survey of Binggeli, Sandage, and Tammann), the NGVS represents an improvement of factor 15 in point-source depth, 25 in surface brightness, and 2 in spatial resolution. The gains over the SDSS imaging of Virgo are comparable.

In this paper, we have described the survey motivation, design, implementation, and science goals. The survey area covers a circular region with radius $5.383(1.55 \mathrm{Mpc}$ assuming a distance to the Virgo cluster of $16.5 \mathrm{Mpc}$ ) centered on M87 (the galaxy at the center of the A subcluster), which partly overlaps with a second circular region of radius $3.334(0.96 \mathrm{Mpc})$ centered on M49 (at the center of the B subcluster). The entire region is tiled with 117 , slightly overlapping MegaCam pointings, each imaging (after stacking multiple dithered exposures) an area of $1^{\circ} \times 1^{\circ}$. The survey has been awarded $810 \mathrm{hr}$ of CFHT time, spread over four years, from 2009 to 2012 . At the time of writing (i.e., at the end of year three), the entire survey area has been covered in $g$, while coverage is nearly complete in $i$ and $z$, and $44 \%$ complete in $u^{*}$. Coverage in $r$ is at present limited to the $4 \mathrm{deg}^{2}$ centered on M87.

To study the outer halos of galaxies, and the extended web of filaments that permeates the intracluster space, the survey must reach surface brightness levels of $\mu_{g} \gtrsim 28 \mathrm{mag} \mathrm{arcsec}^{-2}$. The NGVS has therefore adopted a novel observing strategy, which relies on obtaining, within an uninterrupted sequence, single exposures of a number of fields before re-observing the same fields at a different dithered position. A new data processing pipeline (Elixir-LSB) was developed that constructs "background" frames (dominated by light scattered within the Wide Field Corrector optics) by combining sequential images of different fields, which are then used to correct each individual exposure. Typical residuals in the final, scatteredlight-subtracted images are $0.2 \%$ of the sky background in all filters, corresponding to $\mu_{g} \sim 29 \mathrm{mag} \operatorname{arcsec}^{-2}$. Coincidentally, the observing procedure described above implies that any given field is imaged in any given filter multiple times during the course of several days, but more typically weeks, or even months. This "built-in" time cadence proves to be beneficial for the study of transient phenomena and moving targets, most notably KBOs.

This is the first in an extended series of papers that will present methodological, technical, and/or scientific results from the NGVS.

The NGVS team owes an enormous debt of gratitude to the director and the staff of the Canada-France-Hawaii Telescope, whose dedication, ingenuity, and expertise have helped make the survey a reality. During the past two years, Stephane Arnouts, Daniel Devost, Adam Draginga, Peter Forshay, MaryBeth Laychak, Glenn Morrison, Lisa Wells, and David Woodworth have gone far beyond the call of duty to facilitate the survey's planning and execution, and their help is deeply appreciated. 
This work is supported in part by the French Agence Nationale de la Recherche (ANR) Grant Programme Blanc VIRAGE (ANR10-BLANC-0506-01), and by the Canadian Advanced Network for Astronomical Research (CANFAR) which has been made possible by funding from CANARIE under the Network-Enabled Platforms program.

This research used the facilities of the Canadian Astronomy Data Centre operated by the National Research Council of Canada with the support of the Canadian Space Agency. The authors further acknowledge use of the NASA/IPAC Extragalactic Database (NED), which is operated by the Jet Propulsion Laboratory, California Institute of Technology, under contract with the National Aeronautics and Space Administration, and the HyperLeda database (http://leda.univ-lyon1.fr). This publication has made use of data products from the Sloan Digital Sky Survey (SDSS). Funding for SDSS and SDSS-II has been provided by the Alfred P. Sloan Foundation, the Participating Institutions, the National Science Foundation, the U.S. Department of Energy, the National Aeronautics and Space Administration, the Japanese Monbukagakusho, the Max Planck Society and the Higher Education Funding Council for England.

Finally, our most sincere thanks to the anonymous referee for help in improving the manuscript, and to B.B.K. for inspiration. Facility: CFHT

\section{REFERENCES}

Adami, C., Durret, F., Benoist, C., et al. 2010, A\&A, 509, A81

Abadi, M. G., Navarro, J. F., \& Steinmetz, M. 2006, MNRAS, 365, 747

Aguerri, J. A. L., Gerhard, O. E., Arnaboldi, M., et al. 2005, AJ, 129, 2585

An, D., Pinsonneault, M. H., Masseron, T., et al. 2009, ApJ, 700, 523

Andreon, S. 2008, MNRAS, 386, 1045

Arnaboldi, M. 2005, in AIP Conf. Proc. 804, Planetary Nebulae as Astronomical

Tools, ed. R. Szczerba, G. Stasińska, \& S. K. Gorny (Melville, NY: AIP), 301

Arnaud, M., Pointecouteau, E., \& Pratt, G. W. 2005, A\&A, 441, 893 Arnaboldi, M., Aguerri, J. A. L., Napolitano, N. R., et al. 2002, AJ, 123, 760 Auger, M. W., Treu, T., Bolton, A. S., et al. 2010, ApJ, 724, 511

Baldry, I. K., Balogh, M. L., Bower, R. G., et al. 2006, MNRAS, 373, 469

Baldry, I. K., Glazebrook, K., Brinkmann, J., et al. 2004, ApJ, 600, 681

Balogh, M. L., Navarro, J. F., \& Morris, S. L. 2000, ApJ, 540, 113

Barker, M. K., Ferguson, A. M. N., Cole, A. A., et al. 2011, MNRAS, 410, 504

Bassino, L. P., Muzzio, J. C., \& Rabolli, M. 1994, ApJ, 431, 634

Baum, W. A. 1959, PASP, 71, 106

Becker, R. H., White, R. L., \& Helfand, D. J. 1995, ApJ, 450, 559

Bekki, K., Couch, W. J., Drinkwater, M. J., \& Gregg, M. D. 2001, ApJ, 557, L 39

Bekki, K., Couch, W. J., Drinkwater, M. J., \& Shioya, Y. 2003, MNRAS, 344, 399

Bell, E. F., McIntosh, D. H., Katz, N., \& Weinberg, M. D. 2003, ApJS, 149, 289

Bell, E. F., Wolf, C., Meisenheimer, K., et al. 2004, ApJ, 608, 752

Belokurov, V., Zucker, D. B., Evans, N. W., et al. 2006, ApJ, 642, L137

Bender, R., Burstein, D., \& Faber, S. M. 1992, ApJ, 399, 462

Benjamin, J., Heymans, C., Semboloni, E., et al. 2007, MNRAS, 381, 702

Benson, A. J., Bower, R. G., Frenk, C. S., et al. 2003, ApJ, 599, 38

Bernardi, M., Roche, N., Shankar, F., \& Sheth, R. K. 2011a, MNRAS, 412, L6

Bernardi, M., Roche, N., Shankar, F., \& Sheth, R. K. 2011b, MNRAS, 412, 684 Bertin, E., \& Arnouts, S. 1996, A\&AS, 117, 393

Bertin, E., Mellier, Y., Radovich, M., et al. 2002, in ASP Conf. Proc. 281, Astronomical Data Analysis Software and Systems XI, ed. D. A. Bohlender, D. Durand, \& T. H. Handley (San Francisco, CA: ASP), 228

Bettoni, D., Buson, L. M., \& Galletta, G. 2010, A\&A, 519, A72

Binggeli, B. 1999, in The Radio Galaxy Messier 87, ed. H.-J. Röser \& K. Meisenheimer (Lecture Notes in Physics, Vol. 530; Berlin: Springer), 9 Binggeli, B., Popescu, C. C., \& Tammann, G. A. 1993, A\&AS, 98, 275 Binggeli, B., Sandage, A., \& Tammann, G. A. 1985, AJ, 90, 1681 (VCC) Binggeli, B., Sandage, A., \& Tarenghi, M. 1984, AJ, 89, 64 Binggeli, B., Tammann, G. A., \& Sandage, A. 1987, AJ, 94, 251 Blakeslee, J. P., \& Barber DeGraaff, R. 2008, AJ, 136, 2295 Blakeslee, J. P., Cantiello, M., Mei, S., et al. 2010, ApJ, 724, 657 Blakeslee, J. P., Jordán, A., Mei, S., et al. 2009, ApJ, 694, 556
Blanton, M. R., Hogg, D. W., Bahcall, N. A., et al. 2003, ApJ, 592, 819 Block, D. L., Bournaud, F., Combes, F., et al. 2006, Nature, 443, 832 Böhringer, H., Briel, U. G., Schwarz, R. A., et al. 1994, Nature, 368, 828 Böker, T., Laine, S., van der Marel, R. P., et al. 2002, AJ, 123, 1389 Böker, T., Sarzi, M., McLaughlin, D. E., et al. 2004, AJ, 127, 105 Boselli, A., Boissier, S., Cortese, L., et al. 2006, ApJ, 651, 811 Boselli, A., Boissier, S., Heinis, S., et al. 2011, A\&A, 528, A107 Boselli, A., Eales, S., Cortese, L., et al. 2010, PASP, 122, 261

Boselli, A., \& Gavazzi, G. 2002, A\&A, 386, 124

Boselli, A., \& Gavazzi, G. 2006, PASP, 118, 517

Boselli, A., Gavazzi, G., Franzetti, P., Pierini, D., \& Scodeggio, M. 2000, A\&AS, 142,73

Boselli, A., Iglesias-Páramo, J., Vílchez, J. M., \& Gavazzi, G. 2002, A\&A, 386 , 134

Boselli, A., Sauvage, M., Lequeux, J., Donati, A., \& Gavazzi, G. 2003, A\&A, 406, 867

Boselli, A., Tuffs, R. J., Gavazzi, G., Hippelein, H., \& Pierini, D. 1997, A\&AS, 121,507

Boulade, O., Charlot, X., Abbon, P., et al. 2003, Proc. SPIE, 4841, 72

Bower, R. G., Benson, A. J., Malbon, R., et al. 2006, MNRAS, 370, 645

Boylan-Kolchin, M., Springel, V., White, S. D. M., Jenkins, A., \& Lemson, G. 2009, MNRAS, 398, 1150

Brainerd, T. G., Blandford, R. D., \& Smail, I. 1996, ApJ, 466, 623

Brodie, J. P., \& Strader, J. 2006, ARA\&A, 44, 193

Brown, M. E., Trujillo, C., \& Rabinowitz, D. 2004, ApJ, 612, 645

Brown, M. J. I., Dey, A., Jannuzi, B. T., et al. 2007, ApJ, 654, 858

Bruzual, G., \& Charlot, S. 2003, MNRAS, 344, 1000

Bullock, J. S., \& Johnston, K. V. 2005, ApJ, 635, 931

Cabanac, R. A., Alard, C., Dantel-Fort, M., et al. 2007, A\&A, 461, 813

Caldwell, N. 1983, AJ, 88, 804

Cantiello, M., \& Blakeslee, J. P. 2007, ApJ, 669, 982

Cantiello, M., Blakeslee, J., Raimondo, G., Brocato, E., \& Capaccioli, M. 2007, ApJ, 668, 130

Capetti, A., Kharb, P., Axon, D. J., Merritt, D., \& Baldi, R. D. 2009, AJ, 138, 1990

Cappellari, M., Emsellem, E., Krajnović, D., et al. 2011a, MNRAS, 413, 813

Cappellari, M., Emsellem, E., Krajnović, D., et al. 2011b, MNRAS, 416, 1680

Capuzzo-Dolcetta, R., \& Mastrobuono-Battisti, A. 2009, A\&A, 507, 183

Capuzzo-Dolcetta, R., \& Miocchi, P. 2008, ApJ, 681, 1136

Carollo, C. M., Stiavelli, M., \& Mack, J. 1998, AJ, 116, 68

Cassata, P., Emsellem, E., Krajnović, D., et al. 2007, ApJS, 172, 270

Chen, C.-W., Côté, P., West, A. A., Peng, E. W., \& Ferrarese, L. 2010, ApJS, 191,1

Chiboucas, K., Tully, R. B., Marzke, R. O., et al. 2011, ApJ, 737, 86

Chilingarian, I., Cayatte, V., Revaz, Y., et al. 2009, Science, 326, 1379

Chilingarian, I. V., \& Mamon, G. A. 2008, MNRAS, 385, L83

Chung, A., van Gorkom, J. H., Kenney, J. D. P., Crowl, H., \& Vollmer, B. 2009a, AJ, 138, 1741

Chung, E. J., Rhee, M.-H., Kim, H., et al. 2009b, ApJS, 184, 199

Cleveland, W. S., \& McGill, R. 1984, J. Am. Stat. Assoc., 79, 807

Cole, S., Norberg, P., Baugh, C. M., et al. 2001, MNRAS, 326, 255

Cole, S., Percival, W. J., Peacock, J. A., et al. 2005, MNRAS, 362, 505

Condon, J. J., Cotton, W. D., Greisen, E. W., et al. 1998, AJ, 115, 1693

Conroy, C., Wechsler, R. H., \& Kravtsov, A. V. 2007, ApJ, 668, 826

Conselice, C. J., Gallagher, J. S., III, \& Wyse, R. F. G. 2002, AJ, 123, 2246

Cooper, A. P., Cole, S., Frenk, C. S., et al. 2010, MNRAS, 406, 744

Cortés, J. R., Kenney, J. D. P., \& Hardy, E. 2006, AJ, 131, 747

Cortese, L., Bendo, G. J., Isaak, K. G., Davies, J. I., \& Kent, B. R. 2010, MNRAS, 403, L26

Cortese, L., Catinella, B., Boissier, S., Boselli, A., \& Heinis, S. 2011, MNRAS, 415,1797

Côté, P. 2010, in AIP Conf. Ser. 1240, Hunting for the Dark: the Hidden Side of Galaxy Formation, ed. Victor P. Debattista \& Cristina C. Popescu (Melville, NY: AIP), 331

Côté, P., Blakeslee, J. P., Ferrarese, L., et al. 2004, ApJS, 153, 223

Côté, P., Ferrarese, L., Jordán, A., et al. 2007, ApJ, 671, 1456

Côté, P., Ferrarese, L., Jordán, A., et al. 2008, in IAU Symp. 245, Formation \& Evolution of Galaxy Bulges, ed. M. Bureau, E. Athanassoula, \& B. Barbuy (Cambridge: Cambridge Univ. Press), 395

Côté, P., McLaughlin, D. E., Cohen, J. G., \& Blakeslee, J. P. 2003, ApJ, 591, 850

Côté, P., McLaughlin, D. E., Hanes, D. A., et al. 2001, ApJ, 559, 828

Côté, P., Piatek, S., Ferrarese, L., et al. 2006, ApJS, 165, 57

Coupon, J., Ilbert, O., Kilbinger, M., et al. 2009, A\&A, 500, 981

Croton, D. J., Springel, V., White, S. D. M., et al. 2006, MNRAS, 365, 11

Crowl, H. H., \& Kenney, J. D. P. 2008, AJ, 136, 1623

Cuillandre, J.-C., Magnier, E. A., Isani, S., et al. 2002, Proc. SPIE, 4844, 501 
Dabringhausen, J., Hilker, M., \& Kroupa, P. 2008, MNRAS, 386, 864 Davies, J. I., Baes, M., Bendo, G. J., et al. 2010, A\&A, 518, L48 Davies, J. I., Bianchi, S., Cortese, L., et al. 2012, MNRAS, 419, 3505 Decarli, R., Gavazzi, G., Arosio, I., et al. 2007, MNRAS, 381, 136 Dehnen, W. 1993, MNRAS, 265, 250

De Lucia, G., Poggianti, B. M., Aragn-Salamanca, A., et al. 2007, MNRAS, 374,809

de Vaucouleurs, G. 1953, AJ, 58, 30

de Vaucouleurs, G. 1958, AJ, 63, 253

de Vaucouleurs, G. 1961a, ApJS, 6, 213

de Vaucouleurs, G. 1961b, ApJS, 5, 233

Diemand, J., Kuhlen, M., \& Madau, P. 2007, ApJ, 667, 859

Djorgovski, S., \& Davis, M. 1987, ApJ, 313, 59

Dressler, A., Lynden-Bell, D., Burstein, D., et al. 1987, ApJ, 313, 42

Drinkwater, M. J., Gregg, M. D., Hilker, M., et al. 2003, Nature, 423, 519

Drinkwater, M. J., Jones, J. B., Gregg, M. D., \& Phillipps, S. 2000, PASA, 17, 227

Dubinski, J. 1998, ApJ, 502, 141

Dubinski, J., \& Carlberg, R. G. 1991, ApJ, 378, 496

Duc, P.-A., Braine, J., Lisenfeld, U., Brinks, E., \& Boquien, M. 2007, A\&A, 475,187

Duffau, S. V., Zinn, R., Carraro, G., et al. 2006, RevMexAA Conf. Ser., 26, 70

Durrell, P. R., Ciardullo, R., Feldmeier, J. J., Jacoby, G. H., \& Sigurdsson, S. 2002, ApJ, 570, 119

Dutton, A. A., Conroy, C., van den Bosch, F. C., Prada, F., \& More, S. 2010, MNRAS, 407, 2

Dutton, A. A., Conroy, C., van den Bosch, F. C., et al. 2011, MNRAS, 416, 322 Efstathiou, G., Ellis, R. S., \& Peterson, B. A. 1988, MNRAS, 232, 431

Elliot, J. L., Kern, S. D., Clancy, K. B., et al. 2005, AJ, 129, 1117

Emsellem, E., Cappellari, M., Krajnović, D., et al. 2011, MNRAS, 414, 888

Erben, T., Hildebrandt, H., Lerchster, M., et al. 2009, A\&A, 493, 1197

Erben, T., Schirmer, M., Dietrich, J. P., et al. 2005, Astron. Nachr., 326, 432

Evstigneeva, E. A., Gregg, M. D., Drinkwater, M. J., \& Hilker, M. 2007, AJ, 133,1722

Faber, S. M. 1973a, ApJ, 179, 423

Faber, S. M. 1973b, ApJ, 179, 731

Faber, S. M., \& Jackson, R. E. 1976, ApJ, 204, 668

Faber, S. M., Willmer, C. N. A., Wolf, C., et al. 2007, ApJ, 665, 265

Falcón-Barroso, J., van de Ven, G., Peletier, R. F., et al. 2011, MNRAS, 417, 1787

Feldmeier, J. J., Ciardullo, R., Jacoby, G. H., \& Durrell, P. R. 2003, ApJS, 145, 65

Feldmeier, J. J., Ciardullo, R., Jacoby, G. H., \& Durrell, P. R. 2004, ApJ, 615, 196

Fellhauer, M., Belokurov, V., Evans, N. W., et al. 2006, ApJ, 651, 167

Fellhauer, M., \& Kroupa, P. 2002, MNRAS, 330, 642

Fellhauer, M., \& Kroupa, P. 2005, MNRAS, 359, 223

Ferguson, H. C., \& Sandage, A. 1989, ApJ, 346, L53

Ferguson, H. C., Tanvir, N. R., \& von Hippel, T. 1998, Nature, 391, 461

Ferrarese, L., Côté, P., Dalla, B., et al. 2006a, ApJS, 164, 334

Ferrarese, L., Côté, P., Dalla, B., et al. 2006b, ApJ, 644, L21

Ferrarese, L., \& Ford, H. 2005, Space Sci. Rev., 116, 523

Fraser, W. C., \& Kavelaars, J. J. 2008, Icarus, 198, 452

Fu, L., Semboloni, E., Hoekstra, H., et al. 2008, A\&A, 479, 9

Fuentes, C. I., \& Holman, M. J. 2008, AJ, 136, 83

Gallo, E., Treu, T., Jacob, J., et al. 2008, ApJ, 680, 154

Gallo, E., Treu, T., Marshall, P. J., et al. 2010, ApJ, 714, 25

Gavazzi, G., Boselli, A., Donati, A., Franzetti, P., \& Scodeggio, M. 2003, A\&A, 400,451

Gavazzi, G., Boselli, A., Scodeggio, M., Pierini, D., \& Belsole, E. 1999, MNRAS, 304, 595

Gavazzi, G., Donati, A., Cucciati, O., et al. 2005, A\&A, 430, 411

Gavazzi, G., Franzetti, P., Scodeggio, M., Boselli, A., \& Pierini, D. 2000, A\&A, 361,863

Gavazzi, G., Fumagalli, M., Cucciati, O., \& Boselli, A. 2010, A\&A, 517, A73

Gavazzi, G., Giovanelli, R., Haynes, M. P., et al. 2008, A\&A, 482, 43

Gavazzi, G., Pierini, D., \& Boselli, A. 1996, A\&A, 312, 397

Gebhardt, K., \& Kissler-Patig, M. 1999, AJ, 118, 1526

Geisler, D., Lee, M. G., \& Kim, E. 1996, AJ, 111, 1529

Giovanelli, R., Haynes, M. P., Kent, B. R., et al. 2005, AJ, 130, 2598

Gladders, M. D., \& Yee, H. K. C. 2005, ApJS, 157, 1

Gladman, B., Lawler, S. M., Petit, J. M., et al. 2012, AJ, submitted

Gnedin, O. Y., Kravtsov, A. V., Klypin, A. A., \& Nagai, D. 2004, ApJ, 616, 16

Graham, A. W., Erwin, P., Trujillo, I., \& Asensio Ramos, A. 2003, AJ, 125, 2951

Graham, A. W., \& Guzmán, R. 2003, AJ, 125, 2936

Guo, Q., White, S., Boylan-Kolchin, M., et al. 2011, MNRAS, 413, 101
Gwyn, S. D. J. 2008, PASP, 120, 212

Hanes, D. A., Côté, P., Bridges, T. J., et al. 2001, ApJ, 559, 812

Harris, W. E. 1986, AJ, 91, 822

Harris, W. E. 2001, Saas-Fee Advanced Course 28: Star Clusters (Berlin: Springer), 223

Harris, W. E. 2009, ApJ, 703, 939

Harris, W. E., Whitmore, B. C., Karakla, D., et al. 2006, ApJ, 636, 90

Harsono, D., \& De Propris, R. 2009, AJ, 137, 3091

Haşegan, M., Jordán, A., Côté, P., et al. 2005, ApJ, 627, 203

Haynes, M. P. 2008, in IAU Symp. 244, Dark Galaxies \& Lost Baryons, ed.

J. I. Davies \& M. J. Disney (Cambridge: Cambridge Univ. Press), 83

Haynes, M. P., Giovanelli, R., \& Kent, B. R. 2007, ApJ, 665, L19

Haynes, M. P., Giovanelli, R., Martin, A. M., et al. 2011, AJ, 142, 170

Hildebrandt, H., Muzzin, A., Erben, T., et al. 2011, ApJ, 733, L30

Hildebrandt, H., van Waerbeke, L., \& Erben, T. 2009, A\&A, 507, 683

Hilker, M., Infante, L., Vieira, G., Kissler-Patig, M., \& Richtler, T. 1999, A\&AS, 134,75

Hoekstra, H., Franx, M., Kuijken, K., et al. 2001, ApJ, 548, L5

Hoekstra, H., Mellier, Y., van Waerbeke, L., et al. 2006, ApJ, 647, 116

Hoekstra, H., Yee, H. K. C., \& Gladders, M. D. 2004, ApJ, 606, 67

Hoffman, G. L., Lewis, B. M., \& Salpeter, E. E. 1995, ApJ, 441, 28

Holmberg, E. 1958, Lund Medd. Astron. Obs. Ser. II, 136, 1

Holmberg, E. 1969, Arkiv for Astronomi, 5, 305

Hopkins, P. F., Hernquist, L., Cox, T. J., Keres, D., \& Wuyts, S. 2009, ApJ, 691, 1424

Hubble, E., \& Humason, M. L. 1931, ApJ, 74, 43

Hudson, M. J., Gwyn, S. D. J., Dahle, H., \& Kaiser, N. 1998, ApJ, 503, 531

Huertas-Company, M., Foex, G., Soucail, G., \& Pelló, R. 2009, A\&A, 505, 83

Hughes, T. M., \& Cortese, L. 2009, MNRAS, 396, L41

Huxor, A. P., Phillipps, S., Price, J., \& Harniman, R. 2011, MNRAS, 414, 3557

Hwang, H. S., Lee, M. G., Park, H. S., et al. 2008, ApJ, 674, 869

Ibata, R., Irwin, M., Lewis, G. F., \& Stolte, A. 2001, ApJ, 547, L133

Impey, C., Bothun, G., \& Malin, D. 1988, ApJ, 330, 634

Janowiecki, S., Mihos, J. C., Harding, P., et al. 2010, ApJ, 715, 972

Janz, J., \& Lisker, T. 2008, ApJ, 689, L25

Janz, J., \& Lisker, T. 2009, ApJ, 696, L102

Jerjen, H., \& Binggeli, B. 1997, in ASP Conf. Ser. 116, The Nature of Elliptical Galaxies, ed. M. Arnaboldi, G. S. Da Costa, \& P. Saha (San Francisco, CA: ASP), 239

Jerjen, H., Binggeli, B., \& Barazza, F. D. 2004, AJ, 127, 771

Joachimi, B., Mandelbaum, R., Abdalla, F. B., \& Bridle, S. L. 2011, A\&A, 527, A26

Joachimi, B., \& Schneider, P. 2008, A\&A, 488, 829

Joachimi, B., \& Schneider, P. 2009, A\&A, 507, 105

Johnston, D. E., Sheldon, E. S., Tasitsiomi, A., et al. 2007, ApJ, 656, 27

Jones, J. B., Drinkwater, M. J., Jurek, R., et al. 2006, AJ, 131, 312

Jordán, A., Côté, P., Blakeslee, J. P., et al. 2005, ApJ, 634, 1002

Jordán, A., Côté, P., Ferrarese, L., et al. 2004, ApJ, 613, 279

Jordán, A., McLaughlin, D. E., Côté, P., et al. 2007, ApJS, 171, 101

Jordán, A., Peng, E. W., Blakeslee, J. P., et al. 2009, ApJS, 180, 54

Jordán, A., West, M. J., Côté, P., \& Marzke, R. O. 2003, AJ, 125, 1642

Jurić, M., Ivezić, Ž., Brooks, A., et al. 2008, ApJ, 673, 864

Karick, A. M., Drinkwater, M. J., \& Gregg, M. D. 2003, MNRAS, 344, 188

Kassin, S. A., Weiner, B. J., Faber, S. M., et al. 2007, ApJ, 660, L35

Kavelaars, J. J., Jones, R. L., Gladman, B. J., et al. 2009, AJ, 137, 4917

Keenan, D. W., \& Innanen, K. A. 1975, AJ, 80, 290

Kenney, J. D. P., Koopmann, R. A., Rubin, V. C., \& Young, J. S. 1996, AJ, 111, 152

Kent, B. R. 2008, in ASP Conf. Ser. 395, Frontiers of Astrophysics: A Celebration of NARO's 50th Anniversary, ed. A. H. Bridle, J. J. Condon, \& G. C. Hunt (San Francisco, CA: ASP), 369

Kent, B. R. 2010, ApJ, 725, 2333

Kent, B. R., Giovanelli, R., Haynes, M. P., et al. 2007, ApJ, 665, L15

Kent, B. R., Spekkens, K., Giovanelli, R., et al. 2009, ApJ, 691, 1595

Kim, S., Rey, S.-C., Lisker, T., \& Sohn, S. T. 2010, ApJ, 721, L72

King, I. 1962, AJ, 67, 471

Klypin, A., Hoffman, Y., Kravtsov, A. V., \& Gottlöber, S. 2003, ApJ, 596, 19 Klypin, A., Kravtsov, A. V., Valenzuela, O., \& Prada, F. 1999, ApJ, 522, 82

Klypin, A. A., Trujillo-Gomez, S., \& Primack, J. 2011, ApJ, 740, 102

Kniazev, A. Y., Grebel, E. K., Pustilnik, S. A., et al. 2004, AJ, 127, 704

Koester, B. P., McKay, T. A., Annis, J., et al. 2007, ApJ, 660, 239

Koopmann, R. A., Giovanelli, R., Haynes, M. P., et al. 2008, ApJ, 682, L85

Koopmann, R. A., \& Kenney, J. D. P. 2004, ApJ, 613, 866

Koopmann, R. A., Kenney, J. D. P., \& Young, J. 2001, ApJS, 135, 125

Kormendy, J. 1985, ApJ, 295, 73

Kormendy, J., Fisher, D. B., Cornell, M. E., \& Bender, R. 2009, ApJS, 182, 216

Kravtsov, A. V., Nagai, D., \& Vikhlinin, A. A. 2005, ApJ, 625, 588 
Kundu, A., \& Whitmore, B. C. 2001, AJ, 121, 2950

Kuntschner, H., Emsellem, E., Bacon, R., et al. 2010, MNRAS, 408, 97

Larsen, S. S., Brodie, J. P., Huchra, J. P., Forbes, D. A., \& Grillmair, C. J. 2001, AJ, 121, 2974

Lawrence, A., Warren, S. J., Almaini, O., et al. 2007, MNRAS, 379, 1599

Leauthaud, A., Finoguenov, A., Kneib, J.-P., et al. 2010, ApJ, 709, 97

Leauthaud, A., Tinker, J., Behroozi, P. S., Busha, M. T., \& Wechsler, R. H. 2011, ApJ, 738, 45

Lee, H., Richer, M. G., \& McCall, M. L. 2000, ApJ, 530, L17

Lee, M. G., Park, H. S., \& Hwang, H. S. 2010, Science, 328, 334

Li, Y., Haiman, Z., \& Mac Low, M.-M. 2007, ApJ, 663, 61

Lieder, S., Lisker, T., Hilker, M., Misgeld, I., \& Durrell, P. 2012, A\&A, 538, A69

Lin, H., Yee, H. K. C., Carlberg, R. G., \& Ellingson, E. 1996, J. R. Astron. Soc. Can., 90, 337

Lisker, T., Glatt, K., Westera, P., \& Grebel, E. K. 2006a, AJ, 132, 2432

Lisker, T., Grebel, E. K., \& Binggeli, B. 2006b, AJ, 132, 497

Lisker, T., Grebel, E. K., \& Binggeli, B. 2008, AJ, 135, 380

Lisker, T., Grebel, E. K., Binggeli, B., \& Glatt, K. 2007, ApJ, 660, 1186

Liu, C., Peng, E. W., Jordán, A., et al. 2011, ApJ, 728, 116

Loveday, J., Peterson, B. A., Efstathiou, G., \& Maddox, S. J. 1992, ApJ, 390, 338

Lu, T., Gilbank, D. G., Balogh, M. L., \& Bognat, A. 2009, MNRAS, 399, 1858

Madrid, J. P., Graham, A. W., Harris, W. E., et al. 2010, ApJ, 722, 1707

Magnier, E. A., \& Cuillandre, J.-C. 2004, PASP, 116, 449

Malin, D. 1994, in IAU Symp. 161, Astronomy from Wide-Field Imaging, ed. H. T. MacGillivray (Dordrecht: Kluwer), 567

Malin, D. F. 1979, Nature, 277, 279

Mandelbaum, R., Blake, C., Bridle, S., et al. 2011, MNRAS, 410, 844

Mandelbaum, R., Hirata, C. M., Broderick, T., Seljak, U., \& Brinkmann, J. 2006a, MNRAS, 370, 1008

Mandelbaum, R., Li, C., Kauffmann, G., \& White, S. D. M. 2009, MNRAS, 393, 377

Mandelbaum, R., Seljak, U., Kauffmann, G., Hirata, C. M., \& Brinkmann, J. 2006b, MNRAS, 368, 715

Martínez-Delgado, D., Peñarrubia, J., Jurić, M., Alfaro, E. J., \& Ivezić, Z. 2007, ApJ, 660, 1264

Marzke, R. O., Huchra, J. P., \& Geller, M. J. 1994, ApJ, 428, 43

Masters, K. L., Jordán, A., Côté, P., et al. 2010, ApJ, 715, 1419

McConnachie, A. W., Irwin, M. J., Ibata, R. A., et al. 2009, Nature, 461, 66

McDonald, M., Courteau, S., \& Tully, R. B. 2009, MNRAS, 394, 2022

McDonald, M., Courteau, S., Tully, R. B., \& Roediger, J. 2011, MNRAS, 414, 2055

McLaughlin, D. E. 1999, ApJ, 512, L9

McLaughlin, D. E., King, A. R., \& Nayakshin, S. 2006, ApJ, 650, L37

McLaughlin, D. E., et al. 2012, ApJ, submitted

Mei, S., Blakeslee, J. P., Côté, P., et al. 2007, ApJ, 655, 144

Mei, S., Holden, B. P., Blakeslee, J. P., et al. 2009, ApJ, 690, 42

Mei, S., et al. 2012, ApJ, submitted

Ménard, B., Scranton, R., Fukugita, M., \& Richards, G. 2010, MNRAS, 405, 1025

Merritt, D., \& Szell, A. 2006, ApJ, 648, 890

Mieske, S., Hilker, M., Jordán, A., et al. 2008, A\&A, 487, 921

Mieske, S., Jordán, A., Côté, P., et al. 2006, ApJ, 653, 193

Mieske, S., Jordán, A., Côté, P., et al. 2010, ApJ, 710, 1672

Mihos, J. C. 2004, Clusters of Galaxies: Probes of Cosmological Structure and Galaxy Evolution (Cambridge: Cambridge Univ. Press), 277

Mihos, J. C., Harding, P., Feldmeier, J., \& Morrison, H. 2005, ApJ, 631, L41

Mihos, J. C., Janowiecki, S., Feldmeier, J. J., Harding, P., \& Morrison, H. 2009, ApJ, 698, 1879

Milkeraitis, M., van Waerbeke, L., Heymans, C., et al. 2010, MNRAS, 406, 673

Moore, B., Ghigna, S., Governato, F., et al. 1999, ApJ, 524, L19

Moore, B., Katz, N., Lake, G., Dressler, A., \& Oemler, A. 1996, Nature, 379, 613

Moran, S. M., Ellis, R. S., Treu, T., et al. 2007, ApJ, 671, 1503

More, A., Cabanac, R., More, S., et al. 2012, ApJ, 749, 38

Murante, G., Arnaboldi, M., Gerhard, O., et al. 2004, ApJ, 607, L83

Murante, G., Giovalli, M., Gerhard, O., et al. 2007, MNRAS, 377, 2

Muzzin, A., Wilson, G., Yee, H. K. C., et al. 2009, ApJ, 698, 1934

Nagai, D., \& Kravtsov, A. V. 2005, ApJ, 618, 557

Navarro, J. F., Frenk, C. S., \& White, S. D. M. 1997, ApJ, 490, 493

Navarro, J. F., Hayashi, E., Power, C., et al. 2004, MNRAS, 349, 1039

Navarro, J. F., Ludlow, A., Springel, V., et al. 2010, MNRAS, 402, 21

Neilsen, E. H., Jr., \& Tsvetanov, Z. I. 2000, ApJ, 536, 255

Newberg, H. J., Yanny, B., Cole, N., et al. 2007, ApJ, 668, 221

Norberg, P., Cole, S., Baugh, C. M., et al. 2002, MNRAS, 336, 907

Nulsen, P. E. J., \& Böhringer, H. 1995, MNRAS, 274, 1093
Parker, L. C., Hoekstra, H., Hudson, M. J., van Waerbeke, L., \& Mellier, Y. 2007, ApJ, 669, 21

Parker, L. C., Hudson, M. J., Carlberg, R. G., \& Hoekstra, H. 2005, ApJ, 634, 806

Parry, O. H., Eke, V. R., \& Frenk, C. S. 2009, MNRAS, 396, 1972

Paudel, S., Lisker, T., Kuntschner, H., Grebel, E. K., \& Glatt, K. 2010, MNRAS, 405, 800

Peacock, M. B., Maccarone, T. J., Knigge, C., et al. 2010, MNRAS, 402, 803

Peñarrubia, J., Belokurov, V., Evans, N. W., et al. 2010, MNRAS, 408, L26

Peñarrubia, J., Zucker, D. B., Irwin, M. J., et al. 2011, ApJ, 727, L2

Peng, C. Y., Ho, L. C., Impey, C. D., \& Rix, H.-W. 2002, AJ, 124, 266

Peng, E. W., Côté, P., Jordán, A., et al. 2006a, ApJ, 639, 838

Peng, E. W., Jordán, A., Blakeslee, J. P., et al. 2009, ApJ, 703, 42

Peng, E. W., Jordán, A., Côté, P., et al. 2006b, ApJ, 639, 95

Peng, E. W., Jordán, A., Côté, P., et al. 2008, ApJ, 681, 197

Percival, W. J., Nichol, R. C., Eisenstein, D. J., et al. 2007, ApJ, 657, 645

Phillipps, S., Drinkwater, M. J., Gregg, M. D., \& Jones, J. B. 2001, ApJ, 560, 201

Phillipps, S., Parker, Q. A., Schwartzenberg, J. M., \& Jones, J. B. 1998, ApJ, 493, L59

Poggianti, B. M., Bridges, T. J., Komiyama, Y., et al. 2004, ApJ, 601, 197

Portegies Zwart, S. F., McMillan, S. L. W., \& Gieles, M. 2010, ARA\&A, 48, 431

Power, C., Navarro, J. F., Jenkins, A., et al. 2003, MNRAS, 338, 14

Prugniel, P., Bica, E., Klotz, A., \& Alloin, D. 1993, A\&AS, 98, 229

Purcell, C. W., Bullock, J. S., \& Zentner, A. R. 2007, ApJ, 666, 20

Puzia, T. H., Zepf, S. E., Kissler-Patig, M., et al. 2002, A\&A, 391, 453

Reaves, G. 1956, AJ, 61, 69

Reaves, G. 1962, PASP, 74, 392

Reaves, G. 1983, ApJS, 53, 375

Refregier, A. 2003, MNRAS, 338, 35

Regnault, N., Conley, A., Guy, J., et al. 2009, A\&A, 506, 999

Rhode, K. L., \& Zepf, S. E. 2004, AJ, 127, 302

Rines, K., \& Geller, M. J. 2008, AJ, 135, 1837

Roediger, J. C., Courteau, S., MacArthur, L. A., \& McDonald, M. 2011a, MNRAS, 416, 1996

Roediger, J. C., Courteau, S., McDonald, M., \& MacArthur, L. A. 2011b, MNRAS, 416, 1983

Rossa, J., van der Marel, R. P., Böker, T., et al. 2006, AJ, 132, 1074

Rudick, C. S., Mihos, J. C., Frey, L. H., \& McBride, C. K. 2009, ApJ, 699, 1518

Rudick, C. S., Mihos, J. C., Harding, P., et al. 2010, ApJ, 720, 569

Rudick, C. S., Mihos, J. C., \& McBride, C. 2006, ApJ, 648, 936

Ruff, A. J., Gavazzi, R., Marshall, P. J., et al. 2011, ApJ, 727, 96

Sabatini, S., Davies, J., Scaramella, R., et al. 2003, MNRAS, 341, 981

Sandage, A. 1972, ApJ, 176, 21

Sandage, A., \& Binggeli, B. 1984, AJ, 89, 919

Sandage, A., Binggeli, B., \& Tammann, G. A. 1985, AJ, 90, 1759

Sandage, A., \& Visvanathan, N. 1978, ApJ, 225, 742

Schechter, P. 1976, ApJ, 203, 297

Schindler, S., Binggeli, B., \& Böhringer, H. 1999, A\&A, 343, 420

Schlegel, D. J., Finkbeiner, D. P., \& Davis, M. 1998, ApJ, 500, 525

Schwamb, M. E., Brown, M. E., Rabinowitz, D. L., \& Ragozzine, D. 2010, ApJ, 720, 1691

Scranton, R., Ménard, B., Richards, G. T., et al. 2005, ApJ, 633, 589

Secker, J., Harris, W. E., \& Plummer, J. D. 1997, PASP, 109, 1377

Seth, A., Agüeros, M., Lee, D., \& Basu-Zych, A. 2008, ApJ, 678, 116

Shapley, H., \& Ames, A. 1926, Harv. Coll. Obs. Circ., 294

Shapley, H., \& Ames, A. 1929a, Harv. Coll. Obs. Bull., 864

Shapley, H., \& Ames, A. 1929b, Harv. Coll. Obs. Bull., 865

Shapley, H., \& Ames, A. 1929c, Harv. Coll. Obs. Bull., 866

Shapley, H., \& Ames, A. 1929d, Harv. Coll. Obs. Bull., 868

Shapley, H., \& Ames, A. 1929e, Harv. Coll. Obs. Bull., 873

Shibata, R., Matsushita, K., Yamasaki, N. Y., et al. 2001, ApJ, 549, 228

Sivakoff, G. R., Jordán, A., Sarazin, C. L., et al. 2007, ApJ, 660, 1246

Skrutskie, M. F., Cutri, R. M., Stiening, R., et al. 2006, AJ, 131, 1163

Smith, S. 1936, ApJ, 83, 23

Smith Castelli, A. V., Faifer, F. R., Richtler, T., \& Bassino, L. P. 2008, MNRAS, 391,685

Sommer-Larsen, J., Romeo, A. D., \& Portinari, L. 2005, MNRAS, 357, 478

Spergel, D. N., Bean, R., Doré, O., et al. 2007, ApJS, 170, 377

Spergel, D. N., Verde, L., Peiris, H. V., et al. 2003, ApJS, 148, 175

Springel, V., Wang, J., Vogelsberger, M., et al. 2008, MNRAS, 391, 1685

Springel, V., White, S. D. M., Jenkins, A., et al. 2005, Nature, 435, 629

Stetson, P. B. 1987, PASP, 99, 191

Stetson, P. B. 1989, Presented in V Escola Avançadá de Astrofísica, Aguas de Sǎo Pedro, Brazil, p. 1

Strader, J., Brodie, J. P., Spitler, L., \& Beasley, M. A. 2006, AJ, 132, 2333 
Strader, J., Romanowsky, A. J., Brodie, J. P., et al. 2011, ApJS, 197, 33

Strateva, I., Ivezić, Ž., Knapp, G. R., et al. 2001, AJ, 122, 1861

Tamura, N., Sharples, R. M., Arimoto, N., et al. 2006, MNRAS, 373, 588

Tegmark, M., Eisenstein, D. J., Strauss, M. A., et al. 2006, Phys. Rev. D, 74, 123507

Tollerud, E. J., Bullock, J. S., Strigari, L. E., \& Willman, B. 2008, ApJ, 688, 277

Tonry, J., \& Schneider, D. P. 1988, AJ, 96, 807

Tonry, J. L., Dressler, A., Blakeslee, J. P., et al. 2001, ApJ, 546, 681

Tortora, C., Napolitano, N. R., Cardone, V. F., et al. 2010, MNRAS, 407, 144

Trentham, N., \& Hodgkin, S. 2002, MNRAS, 333, 423

Trümper, J. 1993, Science, 260, 1769

Tully, R. B. 1982, ApJ, 257, 389

Tully, R. B., \& Fisher, J. R. 1977, A\&A, 54, 661

Tully, R. B., \& Fisher, J. R. 1987, Nearby Galaxies Atlas (Cambridge: Cambridge Univ. Press)

Tully, R. B., \& Pierce, M. J. 2000, ApJ, 533, 744

Tully, R. B., Rizzi, L., Shaya, E. J., et al. 2009, AJ, 138, 323

Turner, M., et al. 2012, ApJ, submitted

Urban, O., Werner, N., Simionescu, A., Allen, S. W., \& Böhringer, H. 2011, MNRAS, 414, 2101

van Uitert, E., Hoekstra, H., Velander, M., et al. 2011, A\&A, 534, A14

van Waerbeke, L. 2010, MNRAS, 401, 2093

Van Waerbeke, L., Hildebrandt, H., Ford, J., \& Milkeraitis, M. 2010, ApJ, 723, L13

Villegas, D., Jordán, A., Peng, E. W., et al. 2010, ApJ, 717, 603

Visvanathan, N., \& Sandage, A. 1977, ApJ, 216, 214

Vivas, A. K., Jaffé, Y. L., Zinn, R., et al. 2008, AJ, 136, 1645

Vollmer, B. 2009, A\&A, 502, 427

Vollmer, B., Cayatte, V., Balkowski, C., \& Duschl, W. J. 2001, ApJ, 561, 708
Volonteri, M., Haardt, F., \& Gültekin, K. 2008, MNRAS, 384, 1387

Walcher, C. J., Böker, T., Charlot, S., et al. 2006, ApJ, 649, 692

Walcher, C. J., van der Marel, R. P., McLaughlin, D., et al. 2005, ApJ, 618, 237

Wehner, E. H., \& Harris, W. E. 2006, ApJ, 644, L17

Wen, Z. L., Han, J. L., \& Liu, F. S. 2009, ApJS, 183, 197

West, M. J., \& Blakeslee, J. P. 2000, ApJ, 543, L27

West, M. J., Cote, P., Jones, C., Forman, W., \& Marzke, R. O. 1995, ApJ, 453, L77

West, M. J., Côté, P., Marzke, R. O., \& Jordán, A. 2004, Nature, 427, 31

West, M. J., Jordán, A., Blakeslee, J. P., et al. 2011, A\&A, 528, A115

White, R. L., Becker, R. H., Helfand, D. J., \& Gregg, M. D. 1997, ApJ, 475, 479

Williams, B. F., Ciardullo, R., Durrell, P. R., et al. 2007a, ApJ, 656, 756

Williams, B. F., Ciardullo, R., Durrell, P. R., et al. 2007b, ApJ, 654, 835

Williamson, R., Benson, B. A., High, F. W., et al. 2011, ApJ, 738, 139

Willman, B., Governato, F., Wadsley, J., \& Quinn, T. 2004, MNRAS, 355, 159

Wilson, C. D., Warren, B. E., Irwin, J., et al. 2011, MNRAS, 410, 1409

Wilson, C. D., Warren, B. E., Israel, F. P., et al. 2009a, ApJ, 693, 1736

Wilson, G., Muzzin, A., Lacy, M., et al. 2008, in ASP Conf. Ser. 381, Infrared Diagnostics of Galaxy Evolution, ed. R.-R. Chang, H. I. Teplitz, \& K. Sheth (San Francisco, CA: ASP), 210

Wilson, G., Muzzin, A., Yee, H. K. C., et al. 2009b, ApJ, 698, 1943

Wirth, A., \& Gallagher, J. S., III. 1984, ApJ, 282, 85

Worthey, G. 1994, ApJS, 95, 107

Yasuda, N., Fukugita, M., \& Okamura, S. 1997, ApJS, 108, 417

Yoon, S.-J., Yi, S. K., \& Lee, Y.-W. 2006, Science, 311, 1129

York, D. G., Adelman, J., Anderson, J. E., Jr., et al. 2000, AJ, 120, 1579

Zwicky, F. 1957, Morphological Astronomy (Berlin: Springer)

Zwicky, I. F. 1942, ApJ, 95, 555 